\title{
Multipole expansions of gravitational radiation ${ }^{\star \dagger}$
}

\author{
Kip S. Thorne \\ W. K. Kellogg Radiation Laboratory, California Institute of Technology, Pasadena, California 91125 \\ and Center for Radiophysics and Space Research, Cornell University, Ithaca, New York 14853
}

This paper brings together, into a single unified notation, the multipole formalisms for gravitational radiation which various people have constructed. It also extends the results of previous workers. More specifically:

Part One of this paper reviews the various scalar, vector, and tensor spherical harmonics used in the general relativity literature-including the Regge-Wheeler harmonics, the symmetric, trace-free ("STF") tensors of Sachs and Pirani, the Newman-Penrose spin-weighted harmonics, and the Mathews-Zerilli Clebsch-Gordan-coupled harmonics-which include "pure-orbital" harmonics and "pure-spin" harmonics. The relationships between the various harmonics are presented. Part One then turns attention to gravitational radiation. The concept of "local wave zone" is introduced to facilitate a clean separation of "wave generation" from "wave propagation." The generic radiation field in the local wave zone is decomposed into multipole components. The energy, linear momentum, and angular momentum in the waves are expressed as infinite sums of multipole contributions. Attention is then restricted to sources that admit a nonsingular, spacetime-covering de Donder coordinate system. (This excludes black holes.) In such a coordinate system the multipole moments of the radiation field are expressed as volume integrals over the source. For slow-motion systems, these source integrals are re-expressed as infinite power series in $L / X \equiv$ (size of source)/(reduced wavelength of waves). The slow-motion source integrals are then specialized to systems with weak internal gravity to yield (i) the standard Newtonian formulas for the multipole moments, (ii) the post-Newtonian formulas of Epstein and Wagoner, and (iii) post-postNewtonian formulas.

Part Two of this paper derives a multipole-moment wave-generation formalism for slow-motion systems with arbitrarily strong internal gravity, including systems that cannot be covered by de Donder coordinates. In this formalism one calculates, by any means, the source's instantaneous, near-zone, external gravitational field as a solution of the time-independent Einstein field equations. One then reads off of this near-zone field the source's instantaneous multipole moments; and one plugs those timeevolving moments into the standard radiation formulae given in Part One of this paper.

As building blocks for this formalism, Part Two also does the following things: (1) In the linearized theory of gravity, for the vacuum exterior of an isolated system, it derives the general solution of the field equations (a result due to Sachs, Bergmann, and Pirani). (2) In full nonlinear general relativity, for the vacuum near-zone exterior of an isolated system, it derives the structure of the general solution of the Einstein field equations. That structure is expressed as a sum of products of multipole contributions. It also matches this near-zone field onto an outgoing-wave radiation field. (3) In full nonlinear general relativity, for the vacuum exterior of a stationary isolated system, (a) it presents a definition of multipole moments which meshes naturally with gravitational-wave theory; (b) it introduces the concept of "asymptotically Cartesian and mass centered" (ACMC) coordinate systems; and (c) it shows how to deduce the multipole moments of a source from the form of its metric in an ACMC coordinate system. As an example, the lowest few $(l \leq 3)$ multipole moments of the Kerr metric are computed.

\section{CONTENTS}

PART ONE. THE GENERAL MULTIPOLE-MOMENT FORMALISM

I. Introduction and Summary

A. Motivation and relation to previous work Box 1. A non-comprehensive historical overview of the literature on multipole expansions

B. Outline of this article

Box 2. Key equations in this article: An index 301

C. Notation

II. Spherical Harmonics: Scalar, Vector, and Tensor 308

A. Symmetric, trace-free tensors

308

*Supported in part by the National Science Foundation [AST76-80801 A02 and AST75-21153].

$\dagger$ An earlier version of this paper was preprinted in 1977 by Cornell University in two parts: CRSR 663 "The Generation of Gravitational Waves V. Multipole-Moment Formalisms," and CRSR 664 "The Generation of Gravitational Waves VI. Strong-Field, Slow-Motion Sources."
B. Integrals over a sphere

C. Scalar spherical harmonics

D. Vector spherical harmonics

E. Tensor spherical harmonics

F. Solutions of Laplace's equation

G. Solutions of the wave equation

III. Regions of Spacetime Around an Isolated Source

IV. Multipole Expansion of the Radiation Field

A. Radiation field itself

B. Energy in the waves

C. Linear momentum in the waves

D. Angular momentum in the waves

E. Discussion

V. Multipole Moments Expressed as Integrals Over Source

A. General sources

B. Slow-motion sources

C. Newtonian sources

D. Post-Newtonian sources

E. Post-Post-Newtonian sources and beyond

VI. Conclusion
308

308

309

311

313

314

315

316

316

317

318

319

319 
PART TWO. SLOW-MOTION SOURCES OF GRAVITATIONAL WAVES

VII. Introduction

VIII. Linearized Theory

IX. Dynamical Sources in De Donder Gauge

A. de Donder equations

B. Nonlinearity expansion

C. Linear part of the gravitational field

D. Multiparameter expansion of the near-zone gravitational field

E. Multiparameter expansion of the near-zone gauge conditions and field equations

F. Structure of the general near-zone term in the multiparameter expansion

G. Secular changes of mass, linear momentum, and angular momentum

H. The radiation field

I. Summary

$X$. Stationary Sources in De Donder Gauge

$\mathrm{XI}$. Stationary Sources in an "ACMC" Coordinate

System

A. Motivation

B. Metric in ACMC coordinates

C. Transformations between ACMC coordinate systems

D. Multipole moments of the Kerr metric

XII. Formalism for Calculating Wave Generation

A. The formalism

B. Examples of applications

Acknowledgment

Appendix: Tails of the Gravitational Waves

References

\section{PART ONE. THE GENERAL MULTIPOLE-MOMENT FORMALISM}

\section{INTRODUCTION AND SUMMARY}

\section{A. Motivation and relation to previous work}

Spherical-harmonic multipole expansions are used throughout theoretical physics, astrophysics, and engineering-indeed, wherever one deals with fields, be they electromagnetic, gravitational, hydrodynamical, solidbody....

In linear theories, such as vacuum electromagneticwave theory, life is simple: the field's multipole components evolve independently of each other; there is no coupling. However, in nonlinear theories like general relativity, life is difficult: The multipole components are coupled together by the field equations; they mix with each other as they evolve.

When dealing with scalar fields (e.g., Newton's potential $\Phi)$, life is simple: one can use scalar spherical harmonics $Y_{l m}(\theta, \varphi)$. When dealing with vector fields (e.g., the vector potential $A_{j}$ of electromagnetism), life is more difficult: one must use vector spherical harmonics. And when dealing with tensor fields (e.g., the spatial metric $g_{j k}$ of general relativity), life can be very difficult indeed: one must use tensor spherical harmonics.

General relativity embodies both difficulties: nonlinearity and tensorial fields. Perhaps that is why multipole expansions have been a less popular tool in general relativity than in most field theories.

Despite their infrequent use, general relativistic multipole expansions can be powerful-especially in studies of gravitational radiation. Indeed, their use dates back to Einstein's (1918) original paper on gravitational waves. Einstein used a linear approximation to general relativity ("linearized theory") to show that the waves emitted by slowly changing, weakly gravitating systems are predominantly quadrupolar. Not until 40 years later did anyone compute the other multipole contributions (Sachs and Bergmann, 1958; Pirani, 1964); and those computations were also restricted to weakly gravitating systems (linearized theory), for which nonlinear coupling can be totally ignored.

Since 1956 a number of researchers have used spherical-harmonic expansions for a variety of problems in general relativity, including problems where nonlinearity shows up; see Box 1 (page 302). Unfortunately, the vector and tensor harmonics used have not had any standard form. Each major "school" of relativity theory has invented its own notation and formalism, leaving us today with a legacy that includes Regge-Wheeler (1957) harmonics; the Sachs (1961)-Pirani (1964) symmetric trace-free ("ST F") harmonics; the Mathews (1962)Zerilli (1970) Clebsch-Gordon-coupled harmonics of two types - "pure orbital" and "pure spin"; and the Newman-Penrose (1966) spin-weighted spherical har monics. Moreover, the normalizations used in some of these formalisms are not terribly rational.

Since 1966 people have stopped inventing new formalisms, but there has been no attempt at consolidation. Consolidation is one of the main purposes of this review article. Section II presents each of the spherical-harmonic formalisms (with improved normalizations in some cases), and exhibits the relations between them. Many of those relations are very simple. Section II also discusses the relative advantages of the various formalisms for various types of problems. It then selects two sets of harmonics for use in the remainder of this article: STF and Clebsch-Gordan-coupled (pure-orbital plus pure-spin). The other harmonics (Regge-Wheeler and Newman-Penrose) are trivially expressible in terms of these.

Because the relativistic multipole-expansion literature abounds in a plethora of notations and formalisms, it is difficult to read. Box 1 presents a historical overview of that literature; and the remainder of this article (Secs. III-XII) consolidates the main results of that literature into two unified notations: STF and ClebschGordon-coupled. The two notations are carried along side by side; and the main results are quoted separately in each notation. This is because the notations are so different, and because each has strong advantages in special situations.

In addition to consolidating and reviewing the literature, this article derives a number of new results. Most importantly, it shows that a number of formulas that previously were derived only in linearized theory, or only for systems with Newtonian self-gravity, actually have much wider realms of validity; cf. Box 1 .

This article's presentation is self-contained: the reader is told how to derive every equation presented. However, many pages of calculations are sometimes embodied in a phrase like "insert (4.14') into (4.18) and (4.19), and then integrate with the help of (2.5) and (2.6)." 
The reader should be warned that this article and its author do not aspire to the high level of mathematical rigor and elegance that characterize much of mathematical relativity [e.g., Penrose's $(1964,1968)$ conformal treatment of null infinity, and the Bondi et al. (1962)-Sachs (1964)-Newman and Penrose (1968) treatment of the asymptotic properties of gravitational-wave fields.] Instead, the author seeks a level of rigor that is (i) high enough to give him confidence in the results derived, but also (ii) low enough to permit the treatment of real astrophysical systems in the real, nonasymptotically flat universe. This philosophy shows up most strongly in Sec. III, where the concept of "local wave zone" is introduced to permit a separation of the theory of wave generation from wave propagation. That separation sacrifices the elegant rigor of the BondiSachs-Newman-Penrose approach in order to treat, e.g., sources embedded in galaxies, with neutron stars and black holes nearby and with a distant, inhomogeneous universe that may curve up into closure.

\section{B. Outline of this article}

In large measure this article is a compendium of useful formulas about spherical harmonics and gravitational waves. Box 2 (page 304) is an index designed to help the reader locate specific formulas. Box 2 can also be useful in getting an overview of this article: I suggest that the reader read each paragraph of the following outline, and then before going on to the next paragraph read the corresponding parts of Box 2 and of the Table of Contents.

Section I.C introduces the notation to be used in this article. It is crucial to read Sec. I. C carefully before proceeding on to any other part of the article. This is because the notation is somewhat special-e.g., tensor indices always raised and lowered with flat metric; comma used for flat-space covariant derivative; abbreviations S, STF, T, and TT for special parts of a tensor; capital script letters for ST F tensors; and condensed tensor index notation $S_{B_{l}}$.

Section II is a compendium of formulas about scalar, vector, and tensor spherical harmonics and about their use to solve Laplace's equation and the flat-space wave equation. As discussed above, this section presents and compares the various types of spherical harmonics that abound in the relativity literature, and it selects for use in this article the "STF" harmonics, and the "pure-orbital" and "pure-spin" harmonics. ST F harmonics have not been used much elsewhere; and at first sight they seem somewhat forbidding because of their weird notation. However, I have found them to be a very powerful tool; see, e.g., the calculations in Sec. VIII, and also my use of them (Thorne 1980) in a moment formalism for relativistic radiative transfer.

Section III lays the conceptual foundations for this article's approach to gravitational radiation. In particular, in Sec. III we define the concept of an "isolated source" of gravity waves, and argue that every source in the universe today is probably an isolated source. We then split up spacetime around such a source into "strong-field region," "weak-field near zone," "local wave zone," and "distant wave zone;" and we argue that it is adequate in discussing wave generation to ignore the distant wave zone, and with it to ignore in a cavalier fashion the delicacies of curved-space characteristics versus flat-space characteristics.

Section IV analyzes gravitational radiation in the local wave zone of an isolated source. No restrictions are placed on the strength of the source's internal gravity or on the speed of its internal motions. The gravitational-wave field $h_{j k}^{\mathrm{TT}}$ is expanded in tensor spherical harmonics, with multipole-moment coefficients. Two families of multipole moments are required: "mass moments" (denoted $I^{l m}$ or $\mathcal{G}_{A_{l}}$ ) and "current moments" (denoted $S^{l m}$ or $S_{A_{l}}$ ). The energy, linear momentum, and angular momentum carried by the waves are expressed as sums of multipole contributions. The multipole formulas of this section are useful in a wide variety of gravitational-wave calculations.

Section V restricts attention to sources that admit a nonsingular, spacetime-covering de Donder coordinate system. This probably includes all realistic astrophysical sou rces except black holes. Section V uses a Green's function from Sec. III.G to express the multipole moments $I^{l m}, S^{l m}$ (or $g_{A_{l}}, S_{A_{l}}$ ) of the radiation field as integrals over the source's effective stress-energy tensor $\tau_{\alpha \beta}$. These source integrals are valid for sources with strong internal gravity and fast internal motions; but they are probably not useful in the generic case, because the source's effective stress-energy $\tau_{\alpha \beta}$ includes contributions from the gravitational-wave field $h_{j k}^{\mathrm{TT}}$. In the special case of weak-gravity slow-motion sources, $h_{j k}^{\mathrm{TT}}$ can be removed from $\tau_{\alpha \beta}$, and the source integrals become quite useful. One then can describe the source's internal structure and evolution by Newtonian theory, or post-Newtonian theory, or post-post-Newtonian theory; and the source integrals involve only quantities peculiar to those theories. For example, in the Newtonian case the integrals involve only the mass density and angular momentum densityand, in fact, $\mathscr{g}_{A_{l}}$ is the STF part of the $l$ th moment of the mass distribution; $\delta_{A_{1}}$ is the STF part of the $l$ th moment of the angular momentum distribution.

Part Two of this article (Secs. VII-XII) has two purposes: First, it studies the multipole structure of the full exterior gravitational field of a source (by contrast with previous sections, which studied only the radiation field). For slow-motion sources, this multipole study extends into the "near zone" and examines the nonlinear superposition of the multipoles. This study is the foundation for the second purpose of Part Two: to derive a new multipole-moment formalism for calculating the generation of gravitational waves. This formalism is valid for slow-motion sources with arbitrarily strong internal gravity (e.g., for slowly rotating, deformed neutron stars).

Section VII describes the historical motivation for the new slow-motion wave-generation formalism.

Section XII (end of the article-intervening sections will be described below) presents the details of the new slow-motion wave-generation formalism. In this formalism one calculates, by any means, the source's instantaneous near-zone external gravitational field as a solution of the time-independent Einstein field [Text continues on page 307] 


\section{BOX 1 \\ A NONCOMPREHENSIVE HISTORICAL OVERVIEW OF THE LITERATURE ON MULTIPOLE EXPANSION OF GENERAL RELATIVISTIC GRAVITATIONAL FIELDS}

[Citations in square brackets refer to sections and equations in this article]

A. In Linearized Theory (which totally ignores gravitational forces inside the source of gravitational waves), augmented by "pseudotensor" descriptions of the energy-momentum in the waves:

1. Einstein (1918) derived the "quadrupole-moment formalism" for gravitational-wave generation, including formulas for the metric in the wave zone and for the energy carried off by the waves; but see Ftn. 1 on page 318 [Eqs. (5.27)-(5.30), (4.9), (4.16')].

2. Peters (1964) computed the total angular momentum carried by the quadrupole waves; but see Ftn. 2 on page 319 [Eqs. (4.23)].

3. Papapetrou $(1962,1971)$ extended Einstein's formalism to include currentquadrupole and mass-octupole radiation, and the energy, linear momentum, and angular momentum carried by the waves [Eqs. (5.27)-(5.30); Sec. IV] .

4. Sachs and Bergmann (1958), Sachs (1961), and Pirani (1964) decomposed the general, linearized, vacuum gravitational field into an infinite series of multipole components [Secs. IV.A and VIII] .

5. Mathews (1962), Campbell and Morgan (1971), and Campbell, Macek and Morgan (1977) performed this same decomposition, but in different notation; and they augmented it with expressions for all the multipole moments as integrals over the source [Secs. IV.A, VIII, and V.C].

6. Curtis (1978) used Penrose's twistor formalism to define multipole moments.

B. For Slow-Motion Sources with Newtonian Internal Gravity:

1. Landau and Lifshitz (1941) showed that Einstein's quadrupole-moment formulas are valid for such sources [Eqs. (5.27)-(5.30), (4.9), and (4.16')] .

2. Alternative derivations of the quadrupole-moment formulas-with emphasis on or exclusive attention to radiation-reaction in the source and the reactioninduced changes in the source's energy, angular momentum, and linear momentum-were given by Peres and Rosen (1960), Peres (1962, including current-quadrupole and mass-octupole contributions), Peters (1964), and with vastly improved rigor by Chandrasekhar and Esposito (1970) [Secs. V.C and IV].

3. Burke $(1969,1971)$ developed a radiation-reaction formalism that includes all multipole components.

4. This article extends the wave-generation formalism-including the energy, angular momentum, and linear momentum in the waves-to include all multipole components [Secs. V.C and IV].

C. For Slow-Motion Sources with Post-Newtonian Internal Gravity:

1. Epstein and Wagoner (1975) and Wagoner (1977) developed a wave-generation formalism including mass 2-, 3-, 4-, and 5-poles and current 2-, 3-, and 4poles [Secs. V.D and IV].

D. For Slow-Motion Sources with Post-Post-Newtonian Internal Gravity:

1. This article sketches an Epstein-Wagoner type of wave-generation formalism including all multipole components [Secs. V.E and IV]. 


\section{BOX 1 (Continued)}

E. For Time-Independent Sources with Arbitrarily Strong Gravity:

1. Van der Burg (1968), Geroch (1970), Hansen (1974), Clarke and Sciama (1971), and this article [Secs. X and XI] gave definitions of complete sets of multipole moments ("mass moments" and "current moments") and proofs that they fully determine the structure of the vacuum gravitational field, including its nonlinearities. However, nobody has yet elucidated the relationships between the various definitions of multipole moments.

2. Xanthopoulos (1979) proved that a time-independent system is static (its Killing vector is hypersurface orthogonal) if and only if its current multipole moments all vanish.

3. Hoenselaers, Kinnersley, and Xanthopoulos (1979) developed a method for generating the exact solution to the vacuum Einstein field equations with any arbitrary choice for the values of the multipole moments. For further details see Cosgrove (1980).

4. This article [Secs. IX. D, IX. E, IX. F, and X] develops a "nonlinearity expansion" for the general exact solution to the vacuum field equations-an expansion that exhibits the nonlinear coupling of the multipole moments.

5. Dixon $(1970,1977)$ defined multipole moments for a source that resides in a curved background spacetime rather than being alone in asymptotically flat spacetime.

F. For Slow-Motion Sources with Arbitrarily Strong Internal Gravity:

1. This article shows that the Einstein quadrupole-moment wave-generation formulas, and their generalizations to include higher multipole moments [Secs. A and B of this Box] are valid for such sources [Part Two].

2. Bonnor and Rotenberg (1966), Morgan (1971), and this article [Sec. IX] investigated some features of the nonlinear structure of the near-zone gravitational field.

3. This article [Sec. V.B] expresses the multipole moments as integrals over the source-under the restrictive assumption that the source can be covered by a nonsingular de Donder coordinate system.

G. For Sources with Arbitrary Time Dependence and Arbitrarily Strong Internal Gravity:

1. Janis and Newman (1965), Lamb (1966), Bonnor and Rotenberg (1966), and this article [Sec. IV] gave definitions of complete sets of multipole moments for the radiation field.

2. Bondi, van der Burg, and Metzner (1962) and Sachs (1962) gave an expression for the total energy carried off by the waves and proved theorems about the asymptotic structure of the gravitational field at future null infinity. For reviews of this and later, related work see Sachs (1964) and Penrose $(1964,1968)$.

3. This article [Sec. IV] gives expressions for the total energy, linear momentum, and angular momentum carried off by the waves.

4. Newman and Penrose (1968) and Bardeen and Press (1973) analyzed backscatter and tails in the radiation field.

5. Bontz and Price (1979) and Wagoner (1979) investigated the low-frequency portion of the gravitational-wave spectrum.

6. Ashtekar and Hansen (1978) developed the beginnings of a unified treatment of spatial and null infinity. 


\section{BOX 2}

\section{KEY EQUATIONS IN THIS ARTICLE: AN INDEX}

PART ONE

Sec. I.C. NOTATION

- "STF" subscript notation introduced $\left(s_{A_{l}}, N_{A_{l}}, X_{A_{l}}\right) \ldots$

- "S," "T," "TT," and "STF" parts of tensors defined . . .

$(1.6),(1.12)$

- Commas used as covariant derivatives ..........

$(1.7)-(1.11)$

Secs. II. A and II. B. MANIPULATIONS OF STF TENSORS

- "S" (symmetric) part of an arbitrary tensor evaluated ...

- "STF" part of an arbitrary tensor evaluated . . . . . . . .

- Integrals of STF expressions over a 2 -sphere . . . . . . .

- Expansions of $\left[r^{-1} \otimes_{A l}(t \pm r)\right], A_{l} ! \ldots \ldots \ldots \ldots \ldots$

$(1.13)-(1.15)$

Sec. II. C. SCALAR SPHERICAL HARMONICS

- $Y^{l m}$ defined $\ldots \ldots \ldots \ldots \ldots \ldots \ldots \ldots \ldots \ldots \ldots$

- $Y^{l m}$ orthonormality, parity, and complex conjugation . .

- $\mathcal{Y}_{K_{l}}^{l_{m}}$ defined and related to $Y^{l m} \ldots \ldots \ldots \ldots \ldots$

- $F^{l m}$ and $\mathfrak{F}_{K_{l}}$ (multipole components of an arbitrary scalar field on 2-sphere) defined and related to each other ...

Sec. II. D. VECTOR SPHERICAL HARMONICS

- $\mathrm{Y}^{l^{\prime}, \operatorname{lm}}$ ("pure orbital" Clebsch-Gordan-coupled harmonics)

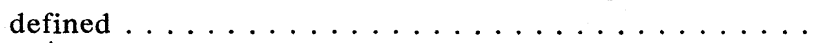

- $\mathrm{Y}^{l^{\prime}, l m}$ orthonormality, parity, and complex conjuga-

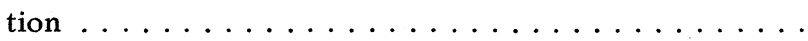

- $\mathbf{Y}^{E, l m}, \mathbf{Y}^{B, l m}, \mathbf{Y}^{R, l m}$ (pure-spin harmonics) defined and related to $Y^{l^{\prime}, l m}$ and to $Y^{l m} \ldots \ldots \ldots \ldots \ldots$

- $\mathbf{Y}^{X, \operatorname{lm}}$ orthonormality, parity, and complex conjuga-

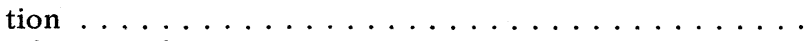

- $\psi^{l m}$ and $\Phi^{l m}$ ("Regge-Wheeler" harmonics) defined in terms of $\mathbf{Y}^{X, \operatorname{lm}} \ldots \ldots \ldots \ldots \ldots \ldots \ldots$

- ${ }_{s} Y^{l m}$ (New man-Penrose spin-weighted harmonics) defined in terms of $\mathbf{Y}^{X, l m} \ldots \ldots \ldots \ldots \ldots \ldots \ldots \ldots$

- STF vector harmonics related to $\mathrm{Y}^{X, \operatorname{lm}} \ldots \ldots \ldots \ldots \ldots$

- STF vector harmonics related to $\mathbf{Y}^{l^{\prime}, l m} \ldots \ldots \ldots \ldots \ldots$

- STF vector-harmonic, algebraic orthonormality rela-

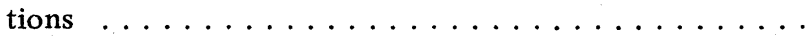

- $E^{l m}$ and $\mathcal{E}_{A_{l}}$ (multipole components of an arbitrary vector field on 2-sphere) defined and related to each other . .

Sec. II. E. TENSOR SPHERICAL HARMONICS

- $\mathbf{T}^{\lambda l^{\prime}, \operatorname{lm}}$ (pure orbital Clebsch-Gordan-coupled harmonics)

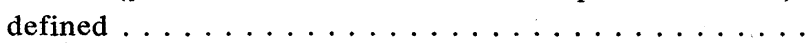

- $\mathrm{T} \lambda l^{\prime}, \mathrm{Im}$ orthonormality, parity, and complex conjuga-

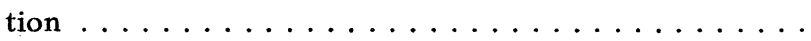

- $\mathbf{T}^{X S, I m}$ (pure spin harmonics) defined and related to $T^{\lambda l^{\prime}, I m}$ and to $Y^{X, I m}$ and to $Y^{l m} \ldots \ldots \ldots \ldots$

- $\mathbf{T}^{X S, l m}$ algebraic and directionality properties .....

- $\mathbf{T}^{X S, l m}$ related to New man-Penrose tetrad components of Riemann tensor of a gravitational wave . . . . . . . .

- $\mathbf{T}^{X S, I m}$ orthonormality, parity, and complex conjuga-

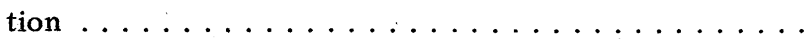




\section{BOX 2 (Continued)}

- $\Phi^{l m} \psi^{l m}, \chi^{l m}$ (Regge-Wheeler harmonics) related to

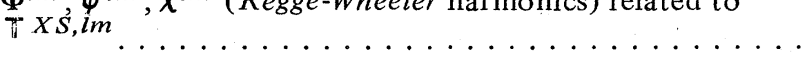

- ${ }_{s} Y^{l m}$ ("Newman-Penrose spin-weighted" harmonics) defined in terms of $\mathbf{T}^{X S, l m} \ldots \ldots \ldots \ldots \ldots \ldots$ (2.38)

- STF tensor harmonics related to $\mathrm{T}^{X S, l m} \ldots \ldots \ldots \ldots$ (2.39)

- STF tensor harmonics related to $\mathrm{T}^{\lambda l^{\prime}, \operatorname{lm}} \ldots \ldots \ldots \ldots$. . . . (2.40) Secs. II. F and II. G. SOLUTIONS OF LAPLACE'S EQUATION AND WAVE EQUATIONS

- $Y^{l m}, \mathbf{Y}^{l^{\prime}, l m}$, and $\mathbf{T}^{\lambda l^{\prime}, l m}$ as eigenfunctions of $\mathbf{L}^{2}$

- Laplace's equation: General Solutions

- in terms of $Y^{l m}, \mathbf{Y}^{l^{\prime}, l m}, \mathbf{T}^{\lambda l^{\prime}, l m} \ldots \ldots \ldots \ldots$ (2.43)

- in terms of STF expansions . . . . . . . . (2.44)

- Wave equations:

- complete sets of "basis" solutions in terms of $Y^{l m}$, $\mathbf{Y}^{l^{\prime}, l m}, \mathbf{T}^{\lambda l^{\prime} l m} \ldots \ldots \ldots \ldots \ldots \ldots \ldots \ldots \ldots \ldots \ldots \ldots \ldots . \ldots \ldots$

- Green's functions in terms of basis solutions . . . . . (2.50)

- general solution in terms of STF functions . . . . . (2.51), (2.53)

Sec. IV. GRAVITATIONAL WAVES FROM ARBITRARY SOURCE, IN LOCAL WAVE ZONE

- $h_{j k}^{\mathrm{TT}}$ expanded

- in pure-spin harmonics $\mathbf{T}^{X S, l m}$ with multipole moments

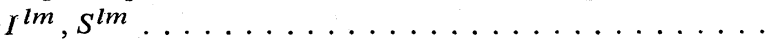

- in STF harmonics with multipole moments

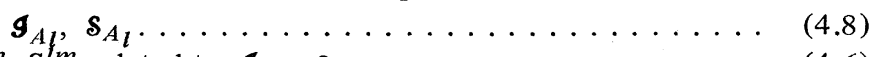

- $I^{l m}, S^{l m}$ related to $\boldsymbol{S}_{A_{l}}, \boldsymbol{s}_{A_{l}} \ldots \ldots \ldots \ldots \ldots$ (4.6), (4.7)

- $I^{l m}, S^{l m}, \boldsymbol{S}_{A_{l}}, \boldsymbol{S}_{A_{l}}$ expressed as angular integrals

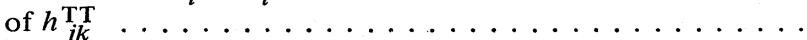

- $d E / d \Omega d t \equiv($ energy flux $)$ in terms of moments $\ldots \ldots \ldots$

- $d E / d t \equiv$ (power radiated) in terms of moments . . . . . .

- $d P_{j} / d t \equiv$ (linear momentum radiated) in terms of mo-

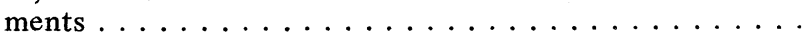

- $d S_{j} / d t \equiv($ angular momentum radiated) in terms of moments . . . . . . . . . . . . . . . (4.23), (4.23') Sec. V. MULTIPOLE MOMENTS EXPRESSED AS INTEGRALS OVER SOURCE IN DE DONDER COORDINATES

- $\tau^{\alpha \beta} \equiv$ (effective stress-energy tensor), which enters into

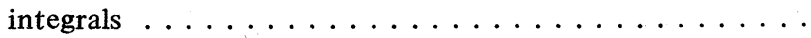

- $I^{l m}, S^{l m}, \boldsymbol{g}_{A_{l}}, \boldsymbol{S}_{A_{l}}$ as integrals over $\tau_{\alpha \beta}$ or over $\rho$ and $v_{j}$

- for arbitrary sources with interior coverable by de Donder coordinates. . . . . . . . . . . . .

- for slow-motion sources with arbitrary internal gravity, and interior coverable by de Donder coordinates:

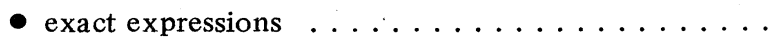

- approximate expressions . . . . . . . . . . .

- errors in approximate expressions . . . . . . . . . 
BOX 2 (Continued)

$\underline{\text { Description }}$

$\underline{\text { Equation Number }}$

- for slow-motion sources with Newtonian internal

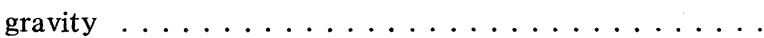

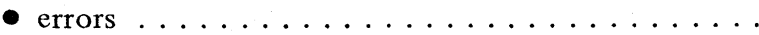

- for slow-motion sources with post-Newtonian internal

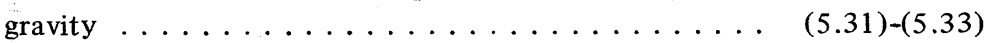

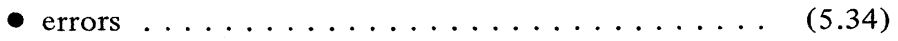

PART TWO. THE GENERAL, VACUUM, EXTERIOR GRAVITATIONAL FIELD FOR VARIOUS TYPES OF SOURCES

Sec. VIII. LINEARIZED SOURCES (NO SELF-GRAVITY) IN LORENTZ GAUGE

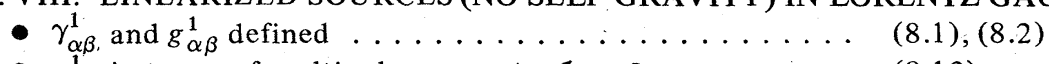

- $\gamma_{\alpha \beta}^{1}$ in terms of multipole moments $\boldsymbol{g}_{A_{l}}, \boldsymbol{s}_{A_{l}} \ldots \ldots \ldots$ (8.12)

- $g_{\alpha \beta}^{1}$ in terms of $\boldsymbol{g}_{A_{l}}, \boldsymbol{S}_{A_{l}} \ldots \ldots \ldots \ldots \ldots \ldots$ (8.13)

Sec. IX. SLOW-MOTION SOURCES WITH ARBITRARY SELF-GRAVITY IN DE DONDER COORDINATES (WHICH NEED NOT COVER SOURCE'S INTERIOR)

- $\bar{h}_{\alpha \beta}$ defined $\ldots \ldots \ldots \ldots \ldots \ldots \ldots \ldots \ldots \ldots \ldots$. $\gamma_{\alpha \beta}^{1}$ (linear part of $\bar{h}_{\alpha \beta}$ ) expressed in terms of multipole

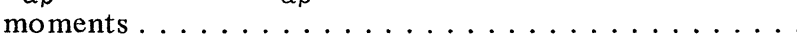

Sec. IX.C

- Near-zone gravitational field:

- $\gamma_{\alpha \beta}^{p n u l}$ defined (multiparameter expansion of $\bar{h}_{\alpha \beta}$ ) . . (9.15), (9.16)

- $g_{\alpha \beta}^{p \text { nul }}$ defined (multiparameter expansion of $g_{\alpha \beta}$ ) . . (9.15), (9.16)

- $\gamma_{\alpha \beta}^{1}$ nul derived from $\gamma_{\alpha \beta}^{1} \ldots \ldots \ldots \ldots \ldots \ldots$ (9.19), (9.20)

- $\gamma_{\alpha \beta}^{p \text { nul }}$ expressed in terms of radius $r$ and time-dependent

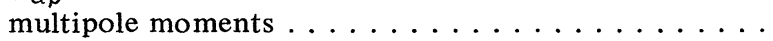

- $g_{\alpha \beta}^{p n u l}$ expressed in terms of radius $r$ and time-dependent multipole moments . . . . . . . . . . .

- Wave-zone gravitational-wave field $\bar{h}_{j k}^{T T}$ in terms of mul-

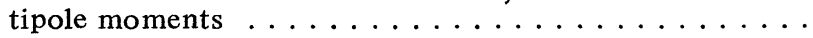

- Wave-zone multipole moments related to near-zone multipole moments: the effect of nonlinearities . . . . . . (9.32)-(9.34)

Sec. X. TIME-INDEPENDENT SOURCES WITH ARBITRARY SELF-GRAVITY IN DE DONDER COORDINATES (WHICH NEED NOT COVER SOURCE'S INTERIOR)

- $g_{\mu \nu}^{1}$ (linear part of metric) expressed in terms of multipole

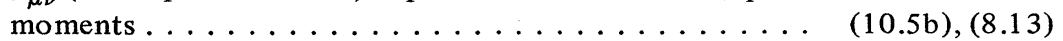

- $g_{\mu \nu}^{p n l}$ defined (multiparameter expansion of $g_{\mu \nu}$ ) . . . . (10.5a), (9.16)

- $g_{\mu \nu}^{p n l}$ expressed in terms of $r$ and multipole moments ... (10.5)

- $g_{\mu \nu}$ expressed as a multipole expansion . . . . . . . (10.6)

Sec. XI. TIME-INDEPENDENT SOURCES WITH ARBITRARY SELF-GRAVITY IN ACMC- $N$ COORDINATES

- $g_{\mu \nu}$ expressed as a multipole expansion . . . . . . . (11.1), (11.4)

- $g_{\mu \nu}$ for Kerr metric in ACMC-2 coordinates . . . . . . (11.26)

- multipole moments of Kerr metric . . . . . . . . . . (11.27), (11.28) 
equations. One then reads off this near-zone field the source's instantaneous multipole moments; and one plugs those time-evolving moments into the radiation formulas of Sec. IV.

The intervening sections, which lay the foundation for this formalism, are the following:

Section VIII restricts attention to "linearized theory" (the linear approximation to general relativity). Using ST F techniques it derives a multipole expansion for the general outgoing-wave solution to the vacuum field equations of linearized theory (external gravitational field of an arbitrary source).

Section IX turns attention to slow-motion stronggravity sources in the full general theory of relativity. The vacuum exterior gravitational field is analyzed using a de Donder coordinate system (Sec. IX.A.). The analysis is based on a "nonlinearity expansion" of the gravitational field (Sec. IX.B)--i.e., an expansion in powers of the gravitation constant $G$ - for which the first-order contribution is the linearized solution of Sec. VIII (see Sec. IX.C). The near-zone gravitational field is studied in Secs. IX.D, IX.E, IX.F, and IX.G using a simultaneous expansion in powers of $G$, in (size of source) $/$ (distance from source) $\equiv L / r$, in (distance from source)/(wavelength of waves) $\equiv L / \lambda$, and in spherical harmonics. This multiparameter expansion gives insight into the general nonlinear structure of the nearzone field. It turns out that the details of the near-zone field are fully determined by two sets of multipole moments: "mass moments" and "current moments." In Sec. IX.H the near-zone field is matched onto the general radiation-zone gravitational-wave field (Sec.IV). This matching shows that, aside from negligible corrections, the multipole moments $g_{A_{l}}, S_{A_{l}}$ that characterize the radiation field are identical to those that characterize the near-zone field.

Section X specializes the near-zone results of Sec.IX to the case of a stationary (time-independent) source. The result is a multipole expansion, in de Donder coordinates, for the general, stationary, asymptotically flat, vacuum solution of the Einstein field equations. The resulting stationary multipole moments are very special: If the source starts to evolve slowly in time and radiate, they become the moments that characterize the radiation field. It is not known whether previous definitions of stationary multipole moments (e.g., Geroch, 1970; Hansen, 1974) have this property.

If one is given a stationary, vacuum solution of the Einstein field equations (e.g., the Kerr metric), how can one compute its multipole moments? One way is to transform to a de Donder coordinate system and then read the moments off the metric coefficients. However, in typical cases (e.g., the Kerr metric) this is prohibitively difficult. Section XI introduces a new, very broad class of coordinate systems in which one can easily read the moments off the metric coefficients. These coordinates are called "asymptotically Cartesian and mass centered to order $N$ " or "ACMC $-N$." As an example, in Sec. XI.D the Kerr metric is transformed to an ACMC -2 coordinate system, and its monopole, dipole, quadrupole, and octupole moments are then read off the form of the metric.

This completes our outline of this article.

\section{Notation}

Throughout this article we use geometrized units $(c=G=1)$; and we use the mathematical notation of flatspace tensor analysis unless otherwise stated. The coordinates $(t, x, y, z)=\left(x^{0}, x^{1}, x^{2}, x^{3}\right)$ are treated in our notation as though they were Cartesian (Minkowskii) coordinates in flat space, and $(t, r, \theta, \phi)$ is the corresponding spherical coordinate system

$$
x=r \sin \theta \cos \phi, y=r \sin \theta \sin \phi, z=r \cos \theta .
$$

Greek indices run from 0 to 3 and Latin from 1 to 3 . We always use orthonormal bases-either

$$
\mathbf{e}_{0}=\frac{\partial}{\partial x^{0}}, \mathbf{e}_{x}=\frac{\partial}{\partial x}, \mathbf{e}_{y}=\frac{\partial}{\partial y}, \mathbf{e}_{z}=\frac{\partial}{\partial z}
$$

or

$$
\mathbf{e}_{0}=\frac{\partial}{\partial x^{0}}, \quad \mathbf{e}_{r}=\frac{\partial}{\partial r}, \mathbf{e}_{\theta}=\frac{1}{r} \frac{\partial}{\partial \theta}, \mathbf{e}_{\phi}=\frac{1}{r \sin \theta} \frac{\partial}{\partial \phi} .
$$

Indices are raised and lowered using the Minkowskii metric, which in either basis (1.2a) or (1.2b) has components

$$
\eta_{00}=-1, \eta_{j k}=\delta_{j k}=(\text { Kronecker delta }) .
$$

Repeated Latin down indices are to be summed as though an $\eta^{j k}=\delta_{j k}$ were present:

$$
A_{j} B_{j}=A_{x} B_{x}+A_{y} B_{y}+A_{z} B_{z}=A_{r} B_{r}+A_{\theta} B_{\theta}+A_{\phi} B_{\phi} .
$$

We shall try, wherever possible, to use down indices for components of vectors and tensors; up indices for everything else. We shall represent the radial vector with length $r$ by $\mathbf{x}$, the unit radial vector by $\mathbf{n}$, and their components by $x_{j}, n_{j}$,

$$
\begin{aligned}
& \mathbf{x} \equiv r \mathbf{e}_{r} ; x_{x}=x, x_{y}=y, x_{z}=z ; \\
& x_{r}=r, x_{\theta}=0, x_{\phi}=0 ; \\
& \mathbf{n}=\mathbf{e}_{r} ; n_{j}=x_{j} / r .
\end{aligned}
$$

Often we shall encounter a sequence of many (say $l$ ) indices on a tensor. For ease of notation we shall abbreviate it as follows:

$$
S_{A_{l}} \equiv S_{a_{1} a_{2} \cdots a_{l}} .
$$

Similarly we shall abbreviate the tensor product of $l$ radial vectors by

$$
N_{B_{l}} \equiv n_{b_{1}} n_{b_{2}} \ldots n_{b_{l}}, X_{B_{l}} \equiv r^{l} N_{B_{l}} \equiv x_{b_{1}} x_{b_{2}} \ldots x_{b_{l}} .
$$

In this abbreviated notation,

$$
S_{A_{l}} N_{B_{l}}=S_{a_{1} a_{2} \cdots a_{l}} n_{b_{1}} n_{b_{2}} \ldots n_{b_{l}} .
$$

Thus, capital subscript letters denote sequences of lower subscript indices, and the number of indices in a sequence is denoted by a subscript to the capital subscript.

We shall denote the symmetric part of a tensor either by parentheses on its indices

$$
\begin{aligned}
S_{a b(c d e)} \equiv & \frac{1}{6}\left(S_{a b c d e}+S_{a b d e c}+S_{a b e c d}\right. \\
& \left.+S_{a b d c e}+S_{a b e d c}+S_{a b c e d}\right)^{\prime},
\end{aligned}
$$

or, when all of the free indices are being symmetrized, by a superscript " $\mathrm{S}$, " 


$$
\left[T_{a b c d e} n_{a} n_{b}\right]^{\mathbb{S}} \equiv T_{a b(c d e)} n_{a} n_{b} .
$$

The fully symmetric and trace-free part of a tensor will be denoted by "STF,"

$$
\begin{aligned}
& {\left[T_{a b c}\right]^{\mathrm{STF}} \equiv T_{(a b c)}-\frac{1}{5} \delta_{a b} T_{(j j c)} } \\
&-\frac{1}{5} \delta_{a c} T_{(j b j)}-\frac{1}{5} \delta_{b c} T_{(a j j)}
\end{aligned}
$$

the transverse part will be denoted by “T,"

$$
\begin{aligned}
{\left[T_{a b c}\right]^{\mathrm{T}} \equiv P_{a i} P_{b j} P_{c k} T_{i j k}, } \\
P_{j k} \equiv \delta_{j k}-n_{j} n_{k} \\
\quad=\text { ("transverse projection tensor"); }
\end{aligned}
$$

and the transverse traceless part will be denoted by “TT,"

$$
\left[T_{a b}\right]^{\mathrm{TT}}=P_{a j} P_{b k} T_{j k}-\frac{1}{2} P_{a b}\left(P_{j k} T_{k j}\right) .
$$

We shall reserve capital script letters to denote tensors that are fully symmetric and trace-free, and that may be functions $t$ and $r$ but are independent (in Cartesian coordinates) of $\theta$ and $\phi$,

$$
B_{A_{l}} \equiv \mathbb{B}_{a_{1} \cdots a_{l}}=\left[\mathbb{B}_{a_{l} \cdots a_{l}}\right]^{\mathrm{STF}} \text {. }
$$

Commas will denote partial derivatives with respect to $(t, x, y, z)$ or flat-space covariant derivatives along the orthonormal basis vectors $\mathbf{e}_{0}, \mathbf{e}_{r}, \mathbf{e}_{\theta}, \mathbf{e}_{\phi}$; $\partial$ will always denote partial derivatives

$$
\begin{aligned}
& A_{x, y}=\partial_{y} A_{x} ; \\
& A_{r, \theta}=r^{-1} \partial_{\theta} A_{r}-\Gamma_{r \theta}^{j} A_{j}=r^{-1} \partial_{\theta} A_{r}-r^{-1} A_{\theta} .
\end{aligned}
$$

That $B_{A_{l}}$ is independent, of $\theta, \phi$ means (see above)

$$
B_{A_{l}, \theta}=B_{A_{l}, \phi}=0 \text {. }
$$

Note that the gradient of the unit radial vector has components

$$
x_{j, k}=\delta_{j k}
$$

and this is true in the orthonormal spherical basis as well as in the Cartesian basis.

For any integer $l$ we shall denote

$$
\begin{aligned}
& l ! \equiv l(l-1) \cdot \cdot 2 \cdot 1, \\
& l ! ! \equiv l(l-2)(l-4) \cdots(2 \text { or } 1) .
\end{aligned}
$$

\section{SPHERICAL HARMONICS: SCALAR, VECTOR, AND TENSOR}

In this section we state, without proof, a number of formulas that are useful in tensorial multipole analyses. The proofs are straightforward, if tedious.

\section{A. Symmetric, trace-free tensors}

Following in the footsteps of Sachs (1961) and Pirani (1964) we shall make extensive use of symmetric tracefree tensors ("STF" tensors). One can calculate the STF part of a tensor which is not ST F by two steps: (i) Construct the symmetric part:

$$
S_{k_{1} \cdots k_{l}} \equiv\left[A_{k_{1} \cdots k_{l}}\right]^{\mathrm{S}}=\frac{1}{l !} \sum_{\pi} A_{k_{\pi}(1) \cdots k_{\pi}(l)} .
$$

Here the summation goes over all $l$ ! permutations $\pi$ of $1, \ldots, l$. (ii) Remove all traces [cf. Eq. (2.44) of Pirani (1964)]:

$\left[A_{k_{1} \circ \cdots k_{l}}\right]^{\mathrm{STF}}=\sum_{n=0}^{[l / 2]} a^{n} \delta_{\left(k_{1} k_{2}\right.} \cdots \delta_{k_{2 n-1} k_{2 n}} S_{\left.k_{2 n+1} \cdots k_{l}\right) j_{1} j_{1} \cdots j_{n^{j}} j_{n}}$,

$a^{n} \equiv(-1)^{n} \frac{l !(2 l-2 n-1) ! !}{(l-2 n) !(2 l-1) ! !(2 n) ! !}$.

Here $[l / 2]$ means the largest integer less than or equal to $l / 2$.

\section{B. Integrals over a sphere}

The following integrals are useful when doing multipole calculations:

$$
\begin{aligned}
& \frac{1}{4 \pi} \int N_{A_{2 l+1}} d \Omega=0 \\
& \frac{1}{4 \pi} \int N_{A_{2 l}} d \Omega=\left(\frac{1}{2 l+1}\right) \delta_{\left(a_{1} a_{2}\right.} \cdots \delta_{\left.a_{2 l-1} a_{2 l}\right)} .
\end{aligned}
$$

[Recall definition (1.6b) of $N_{A_{l}}$.] The completely symmetrized product of Kronecker deltas has the form

$$
\begin{aligned}
& \delta_{\left(a_{1} a_{2}\right.} \cdots \delta_{\left.a_{2 l-1} a_{2 l}\right)}= \\
& =\frac{1}{(2 l-1) ! !} \sum_{j_{2}, j_{4}, \cdots, j_{2 k}} \delta_{a_{1} a_{j_{2}}} \delta_{a_{j_{3}} a_{j_{4}}} \cdots \delta_{a_{j_{2 l-1} a_{j_{2} l}}},
\end{aligned}
$$

where

$j_{2}$ is summed from 2 to $2 l$,

$j_{3}$ is the smallest integer not equal to 1 or $j_{2}$,

$j_{4}$ is summed over all integers, 2 to $2 l$, not equal to $j_{2}$ or $j_{3}$,

$j_{5}$ is the smallest integer not equal to 1 or $j_{2}$ or $j_{3}$ or $j_{4}$,

From Eqs. (2.3) and (2.4) it follows that, for any two ST F tensors $Q$ and $B$,

$\frac{1}{4 \pi} \int\left(Q_{A_{p}} N_{A_{p}}\right)\left(\Theta_{B_{q}} N_{B_{q}}\right) d \Omega$

$=0$ if $Q$ and $B$ have different rank, $p \neq q$

$=\frac{p !}{(2 p+1) ! !} \mathcal{Q}_{A_{p}} \mathbb{B}_{A_{p}}$ if $p=q ;$

$\frac{1}{4 \pi} \int n_{i}\left(Q_{A_{p}} N_{A_{p}}\right)\left(Q_{B_{q}} N_{B_{q}}\right) d \Omega$

$=0$ unless $Q$ and $B$ differ in rank by 1

$=\frac{p !}{(2 p+1) ! !} Q_{i A_{p-1}}{\mathbb{A}_{A-1}}_{p-p}$ if $q=1$.

\section{Scalar spherical harmonics}

The usual representation of scalar spherical harmonics is in terms of complex functions of $\theta$ and $\phi$, 


$$
\begin{aligned}
Y^{l m} & =C^{l m} e^{i m \phi} P^{l m}(\cos \theta) & & \text { for } m \geqslant 0, \\
& =C^{l m}\left(e^{i \phi} \sin \theta\right)^{m} \sum_{j=0}^{[(l-m) / 2]} a^{l m j}(\cos \theta)^{l-m-2 j} & & \text { for } m \geqslant 0, \\
& =(-1)^{m} Y^{l l m l *} & & \text { for } m<0 .
\end{aligned}
$$

Here * denotes complex conjugation; $[(l-m) / 2]$ means "the largest integer less than or equal to $(l-m) / 2$ "; and

$$
\begin{aligned}
& C^{l m} \equiv(-1)^{m}\left(\frac{2 l+1}{4 \pi} \frac{(l-m) !}{(l+m) !}\right)^{1 / 2}, \\
& a^{l m j} \equiv \frac{(-1)^{j}}{2 l j !(l-j) !} \frac{(2 l-2 j) !}{(l-m-2 j) !} .
\end{aligned}
$$

These scalar harmonics are orthonormal

$$
\int Y^{l m} Y^{l^{\prime} m^{\prime} *} d \Omega=\delta_{l l^{\prime}} \delta_{m m^{\prime}} ;
$$

they have parity $\pi=(-1)^{l}$ ("electric-type" parity); and under complex conjugation they transform as

$$
Y^{l m *}=(-1)^{m} Y^{l-m} .
$$

The set of all symmetric trace-free tensors of rank $l$ ("STF- $l$ tensors") generates an irreducible representation of the rotation group, of weight $l$ [Gel'fand $e t a l$. (1963), Courant and Hilbert (1953)]. Hence, there exists a one-to-one mapping between them and the spherical harmonics of order $l$; see Pirani (1964), pp. 289-290. To exhibit that mapping one expresses the Cartesian components of the unit radial vector $\mathbf{n}$ in the form

$$
n_{x}+i n_{y}=e^{i \phi} \sin \theta, \quad n_{z}=\cos \theta
$$

and one then inserts these expressions into Eq. (2.7), obtaining

$$
Y^{l m}(\theta, \phi)=\mathcal{Y}_{K_{l}}^{l m} N_{K_{l}}
$$

Here $\mathcal{Y}_{K_{l}}^{l m}$ is the following (location-independent) STF- $l$ tensor:

$$
\begin{aligned}
& Y_{k_{1} \cdots k_{l}}^{l m} \equiv C^{l m} \sum_{j=0}^{[(l-m) / 2]} a^{l m j}\left(\delta_{\left(k_{1}\right.}^{1}+i \delta_{\left(k_{1}\right.}^{2}\right) \cdots\left(\delta_{k_{m}}^{1}+i \delta_{k_{m}}^{2}\right) \\
& \times \delta_{k_{m+1}}^{3} \cdots \delta_{k_{l-2 j}}^{3}\left(\delta_{k_{l-2 j+1}}^{a_{1}} \delta_{k_{l-2 j+2}}^{a_{1}}\right) \cdots\left(\delta_{k_{l-1}}^{a_{j}} \delta_{k_{l}}^{a_{j}}\right) \\
& Y_{k_{1} \cdots k_{l}}^{l m}=(-1)^{m}\left(Y_{k_{1} \cdots k_{l}}^{l l m l}\right) * \text { for } m<0, \text { for } m \geqslant 0
\end{aligned}
$$

where $\delta_{k_{s}}^{a} i$ is the Kronecker delta.

The tensors $\mathcal{Y}_{K_{l}}^{l m}$ with $-l \leqslant m \leqslant+l$ serve two roles: first, they generate the spherical harmonics of order $l$ [Eq. (2.11)]; second, they form a basis for the $(2 l+1)$ dimensional vector space of STF- $l$ tensors; i.e., any STF- $l$ tensor $\mathcal{F}$ can be expanded as

$$
\mathcal{F}_{K_{l}}=\sum_{m=-l}^{l} F^{l m} \mathcal{Y}_{K_{l}}^{l m}
$$

The tensor components $\mathcal{F}_{K_{l}}$ are real if and only if $F^{i-m}$ $=(-1)^{m} F^{i m *}$. Expansion (2.13a) can be inverted as follows:

$$
\begin{aligned}
F^{l m} & =\int \sum_{m^{\prime}=-l}^{l}\left(F^{l m^{\prime}} Y^{l m^{\prime}}\right) Y^{l m *} d \Omega=\int \mathcal{F}_{K_{l}} N_{K_{l}} Y^{l m *} d \Omega \\
& =4 \pi \frac{l !}{(2 l+1) ! !} \Im_{K_{l}} Y_{K_{l}}^{l m *} .
\end{aligned}
$$

Here the integral is over a sphere surrounding the origin, and the integral was performed with the help of Eqs. (2.11) and (2.5).

In practical calculations one can use spherical harmonics and STF- $l$ tensors interchangeably: Consider any sphere centered on the coordinate origin, and on that sphere consider any scalar function $f(\theta, \phi)$. One can expand $f(\theta, \phi)$ in spherical harmonics with complexnumber expansion coefficients

$$
f(\theta, \phi)=\sum_{l=0}^{\infty} \sum_{m=-l}^{l} F^{l m} Y^{l m}(\theta, \phi) ;
$$

alternatively, one can expand it in powers of the unit radial vector $\mathrm{n}$, with coefficients that are $\mathrm{STF}-l$ tensors

$$
f(\theta, \phi)=\sum_{l=0}^{\infty} \mathcal{F}_{K_{l}} N_{K_{l}} \cdot
$$

The expansion coefficients of the two schemes are related by Eqs. (2.13a) and (2.13b).

\section{Vector spherical harmonics}

Several different conventions for vector spherical harmonics exist in the literature. That of Rose [(1955), p. 22], Edmonds [(1957), p. 82], and Mathews (1962) is the most closely tied to the rotation group. It is obtained by coupling scalar harmonics of order $l^{\prime}$ to the basis vectors

$$
\boldsymbol{\xi}^{0} \equiv \mathbf{e}_{z}, \quad \xi^{ \pm 1} \equiv \mp\left(\mathbf{e}_{x} \pm i \mathbf{e}_{y}\right) / \sqrt{2}
$$

(which transform under an irreducible representation of order 1), ther eby obtaining harmonics $\mathrm{Y}^{l^{\prime}, l m}$ that transform under a representation of order $l=l^{\prime} \pm(1$ or 0$)$,

$$
\begin{gathered}
\mathrm{Y}^{l^{\prime}, l m}(\theta, \phi)=\sum_{m^{\prime}=-l^{\prime}}^{l^{\prime}} \sum_{m^{\prime \prime}=-1}^{1}\left(1 l^{\prime} m^{\prime \prime} m^{\prime} \mid l m\right) \xi^{m^{\prime \prime}} Y^{l^{\prime} m^{\prime}}, \\
l^{\prime}=l \pm(1 \text { or } 0) .
\end{gathered}
$$

Here $\left(l^{\prime \prime} l^{\prime} m^{\prime \prime} m^{\prime} \mid l m\right)$ are Wigner (Clebsch-Gordan) coefficients. (Edmonds and Mathews use the notation $\mathrm{Y}_{l l^{\prime} m}$; Rose uses $\left.\mathbf{T}_{l l^{\prime}}^{m}\right)$ We shall call $\mathrm{Y}^{l^{\prime}, l m}$ and the corresponding tensor harmonics (Sec. II.E) "pure-orbital harmonics" because they are eigenfunctions of the orbital angular momentum operator $\mathrm{L}^{2}$ [Eq. (2.42b)]. For fixed $l^{\prime}$ and $l$ they transform among each other according to an ir reducible representation of order $l$. These harmonics are orthonormal

$$
\int \mathrm{Y}^{\imath, L M} \cdot \mathrm{Y}^{l^{\prime}, L^{\prime} M^{\prime} *} d \Omega=\delta_{l l^{\prime}}, \delta_{L L^{\prime}}, \delta_{M M^{\prime}} ;
$$

$\mathrm{Y}^{l^{\prime}, l m}$ has parity $\pi=(-1)^{l^{\prime \prime}+1}$ [which means that, because the Cartesian basis vectors $\mathbf{e}_{x}, \mathbf{e}_{y}, \mathbf{e}_{z}$ reverse direction under parity inversions, the Cartesian components satisfy $\left.Y_{j}^{l^{\prime}, l m}(-\mathbf{n})=-(-1)^{l^{\prime}+1} Y_{j}^{l^{\prime}, l m}(\mathbf{n})\right]$; and under complex conjugation it transforms as

$$
\mathrm{Y}^{l^{\prime}, l m *}=(-1)^{l^{\prime}+l+m+1} \mathrm{Y}^{l^{\prime}, l-m} \text {. }
$$

These pure-orbital vector harmonics are nicely re- 
lated to solutions of Laplace's equation and the vector wave equation (Secs. II.F and II.G). However, they are not optimally designed for describing radiation, in the radiation zone, because $\mathrm{Y}^{l \pm 1, l m}$ is neither purely radial nor purely transverse. The following "pure-spin vector harmonics" are better suited for describing radiation:

$$
\begin{aligned}
\mathrm{Y}^{E, l m} & =(2 l+1)^{-1 / 2}\left[(l+1)^{1 / 2} \mathbf{Y}^{l-1, l m}+l^{1 / 2} \mathrm{Y}^{l+1, l m}\right] \\
& =[l(l+1)]^{-1 / 2} \gamma \nabla Y^{l m}=-\mathrm{n} \times \mathrm{Y}^{B, l m}, \\
\mathrm{Y}^{B, l m} & =i \mathbf{Y}^{l, l m}=i[l(l+1)]^{-1 / 2} \mathrm{~L} Y^{l m}=\mathrm{n} \times \mathrm{Y}^{E, l m}, \\
\mathrm{Y}^{R, l m} & =(2 l+1)^{-1 / 2}\left[l^{1 / 2} \mathbf{Y}^{l-1, l m}-(l+1)^{1 / 2} \mathrm{Y}^{l+1, l m}\right] \\
& =\mathrm{n} Y^{l m} .
\end{aligned}
$$

Here $\boldsymbol{\nabla}$ is the gradient operator of Euclidean threespace, and $L$ is the angular momentum operator

$$
\mathbf{L} \equiv(1 / i) \mathbf{x} \times \boldsymbol{\nabla} .
$$

$\mathrm{Y}^{E, l m}$ and $\mathrm{Y}^{B, l m}$ are purely transverse; $\mathrm{Y}^{R, l m}$ is purely radial; $\mathrm{Y}^{E, l m}$ and $\mathrm{Y}^{R, l m}$ have "electric-type parity" $\pi=(-1)^{l} ; \mathrm{Y}^{B, l m}$ has “magnetic-type parity” $\pi=(-1)^{l+1}$; these Y's are orthonormal

$$
\int \mathbf{Y}^{J, l m} \cdot \mathbf{Y}^{J \cdot}, l^{\prime} m^{\prime} * d \Omega=\delta_{J J}, \delta_{l l^{\prime}} \delta_{m m^{\prime}} ;
$$

and their complex conjugates are

$$
\mathrm{Y}^{J, l m *}=(-1)^{m} \mathrm{Y}^{J, l-m} \text {. }
$$

[The factor $i$ was included in Eq. (2.18b) in order to produce this complex conjugate relationship.] We call these harmonics "pure spin" because they enter in pure form into the description of the polarization of purespin zero-rest-mass vector fields $\left(\mathrm{Y}^{E, l m}\right.$ and $\mathrm{Y}^{B, l m}$ for transverse spin-one-i.e., electromagnetism; $\mathbf{Y}^{R, l m}$ for longitudinal spin-zero). These pure-spin vector harmonics are intimately related to the Regge-Wheeler (1957) vector harmonics:

$$
\begin{aligned}
& \mathbf{Y}^{E, l m}=[l(l+1)]^{-1 / 2} \Psi^{l m}, \\
& \mathbf{Y}^{B, l m}=[l(l+1)]^{-1 / 2} \Phi^{l m}, \\
& \mathbf{Y}^{R, l m}=\mathbf{n} Y^{l m} ;
\end{aligned}
$$

and also to the Newman-Penrose (1966) spin-weighted spherical harmonics ${ }_{s} Y^{l m}$. [for details of which see Goldberg et al. (1967)]:

$$
\begin{aligned}
& \mathrm{Y}^{E, l m}=2^{-1 / 2}\left({ }_{-1} Y^{l m} \mathrm{~m}-{ }_{1} Y^{l m} \mathrm{~m}^{*}\right) \\
& \mathrm{Y}^{B, l m}=-2^{-1 / 2} i\left({ }_{-1} Y^{l m} \mathrm{~m}+{ }_{1} Y^{l m} \mathrm{~m}^{*}\right) \\
& \mathrm{Y}^{R, l m}={ }_{0} Y^{l m} \mathrm{n}, \\
& \mathrm{m} \equiv 2^{-1 / 2}\left(\mathrm{e}_{\theta}+i \mathrm{e}_{\phi}\right), \quad \mathrm{m}^{*}=2^{-1 / 2}\left(\mathrm{e}_{\theta}-i \mathrm{e}_{\phi}\right) .
\end{aligned}
$$

The STF version of vector spherical harmonics can be obtained by inserting expression $(2.11)$ for $Y^{l m}$ into the second expression in each of Eqs. $(2.18 \mathrm{a}-2.18 \mathrm{c})$ for $\mathrm{Y}^{E, \imath m}, \mathrm{Y}^{B, l m}$, and $\mathrm{Y}^{R, \imath m}$,

$$
\begin{gathered}
Y_{j}^{E, l m}=[l /(l+1)]^{1 / 2}\left[\mathcal{Y}_{j A}^{l m} N_{A_{l-1}}\right]^{T} \\
{\left[\pi=(-1)^{l}\right],} \\
Y_{j}^{B, l m}=[l /(l+1)]^{1 / 2} \epsilon_{j p q} n_{p} \mathcal{Y}_{\alpha A}^{l m} N_{A_{l-1}} \\
{\left[\pi=(-1)^{l+1}\right],} \\
Y_{j}^{R, l m}=n_{j} \mathcal{Y}_{A_{l}}^{l m} N_{A_{l}} \quad\left[\pi=(-1)^{l}\right] .
\end{gathered}
$$

Here $\epsilon_{j p q}$ is the Levi-Civita tensor (antisymmetric symbol), and the superscript $T$ means "transverse part of" [Eq. (1.9)]。 By comparing with Eqs. (2.18) one can derive the relationship between the pure-orbital harmonics and the STF harmonics

$$
\begin{aligned}
Y_{j}^{l-1, l m}= & {[l /(2 l+1)]^{1 / 2} \mathcal{Y}_{j A_{l-1}}^{l m} N_{A_{l-1}}, } \\
Y_{j}^{l, l m}=- & -i[l /(l+1)]^{1 / 2} \epsilon_{j p q} n_{p} \mathcal{Y}_{q A_{l-1}^{l m}}^{l m} N_{A_{l-1}}, \\
Y_{j}^{l+1, l m}= & -[(2 l+1) /(l+1)]^{1 / 2} \\
& \times\left\{n_{j} \mathcal{Y}_{A_{l}}^{l m} N_{A_{l}}-[l /(2 l+1)] \mathcal{Y}_{j A_{l-1}^{l m}}^{l m} N_{A_{l-1}}\right\} .
\end{aligned}
$$

An arbitrary vector field $\mathrm{V}(\theta, \phi)$ can be expanded in terms of pure-orbital harmonics or pure-spin harmonics or Regge-Wheeler harmonics or spin-weighted harmonics; and one can read off the relationships between the expansion coefficients by examining the relationships between the harmonics themselves. For example, the pure-spin and STF expansions have the forms

$$
\begin{aligned}
& V_{j}=\sum_{l, m}\left(E^{l m} Y_{j}^{E, l m}+B^{l m} Y_{j}^{B, l m}+R^{l m} Y_{j}^{R, l m}\right), \\
& V_{j}=\sum_{l}\left(\left[\mathcal{E}_{j A_{l-1}} N_{A_{l-1}}\right]^{T}+\epsilon_{j p q} n_{p} B_{q A_{l-1}} N_{A_{l-1}}+n_{j} R_{A_{l}} N_{A_{l}}\right) ;
\end{aligned}
$$

and by comparing with Eqs. (2.23) one deduces that

$$
\begin{aligned}
& \mathcal{E}_{A_{l}}=\left(\frac{l}{l+1}\right)^{1 / 2} \sum_{m} E^{l m} \mathcal{Y}_{A_{l}}^{l m}, \\
& B_{A_{l}}=\left(\frac{l}{l+1}\right)^{1 / 2} \sum_{m} B^{l m} \mathcal{Y}_{A_{l}}^{l m}, \\
& {A_{A_{l}}}^{l m} \sum_{m} R^{l m} \mathcal{Y}_{A_{l}}^{l m} .
\end{aligned}
$$

One can invert these relations using Eqs. (2.13).

In calculations with STF spherical harmonics the following identities are useful:

$$
\begin{aligned}
& \mathcal{Y}_{A_{l}}^{l m *} \mathcal{Y}_{A_{l}}^{l m^{\prime}}=\frac{1}{4 \pi} \frac{(2 l+1) ! !}{l !} \delta_{m^{\prime} m} \\
& \mathcal{Y}_{A_{l}}^{l m} * \mathcal{Y}_{j A_{l}}^{(l+1)(\mu+m)}=\frac{1}{4 \pi} \frac{(2 l+3) ! !}{(l+1) !}\left(\frac{l+1}{2 l+3}\right)^{1 / 2}(1 l \mu m \mid l+1 \mu+m) \xi_{j}^{\mu} \\
& \text { if } \mu=0 \text { or } \pm 1 \\
& =0 \text { if } \mu \neq 0 \text { or } \pm 1 \text {, }
\end{aligned}
$$

$\epsilon_{j p q} \mathcal{Y}_{p A}^{l m} * Y_{l-1 .}^{l(m-1)}=\frac{i}{4 \pi} \frac{(2 l+1) ! !}{l ! 2 l}[2(l+m)(l-m+1)]^{1 / 2} \xi_{j}^{-1}$,

$\epsilon_{j p q} \mathcal{Y}_{p A}^{l m} * \mathcal{Y}_{q A}^{l m}=\frac{i}{4 \pi} \frac{(2 l+1) ! !}{l !} \frac{m}{l} \xi_{j}^{0}$,

$\epsilon_{j p q} \mathcal{Y}_{p A}^{l m *} \mathcal{Y}_{q A}^{l(m+1)}=\frac{-i}{4 \pi} \frac{(2 l+1) ! !}{l ! 2 l}[2(l-m)(l+m+1)]^{1 / 2} \xi_{j}^{1}$,

$\epsilon_{j p q} \mathcal{Y}_{p A}^{l m *} \mathcal{Y}_{q-1}^{l(m+\mu)}=0$ if $\mu \neq 0$ or \pm 1

Equation (2.26a) can be derived by combining the orthonormality relation for scalar harmonics with Eqs. (2.11) and (2.5); Eq. (2.26b) follows from Eqs. (2.6), (2.23c), (2.18c), (2.11), (2.16), and orthonormality for scalar harmonics; Eqs. (2.26c)-(2.26f) follow from Eqs. 
(2.5), (2.24a), (2.16), (2.15), orthonormality for scalar harmonics, and algebraic expressions for the relevant Clebsch-Gordan coefficients.

\section{E. Tensor spherical harmonics}

Mathews (1962) has constructed a set of "pure-orbital" tensor spherical harmonics which, like the $\mathrm{Y}^{l^{\prime}, l m}$, are closely tied to the rotation group. He first couples the basis vectors $\xi^{m}$ to obtain five symmetric basis tensors $t^{m}$, which transform among each other under an irreducible representation of the rotation group of or der 2,

$$
\mathbf{t}^{m}=\sum_{m^{\prime}=-1}^{1} \sum_{m^{\prime \prime}=-1}^{1}\left(11 m^{\prime} m^{\prime \prime} \mid 2 m\right) \xi^{m^{\prime}} \otimes \xi^{m^{\prime \prime}},
$$

and a single basis tensor - the unit tensor (equal to $3^{-1 / 2}$ times the Euclidean metric) - which gives a representation of order zero

$$
3^{-1 / 2} \delta=-\sum_{m^{\prime}=-1}^{1} \sum_{m^{\prime \prime}=-1}^{1}\left(11 m^{\prime} m^{\prime \prime} \mid 00\right) \xi^{m^{\prime}} \otimes \xi^{m^{\prime \prime}} .
$$

The analogous tensors that give a representation of 'order 1 are antisymmetric, and are thus of no interest for gravitational-wave theory. See Zerilli (1970) for details. In terms of Cartesian basis vectors $\mathrm{e}_{x}, \mathrm{e}_{y}, \mathrm{e}_{z}$ these symmetric basis tensors are

$$
\begin{aligned}
& \mathrm{t}^{ \pm 2}=\frac{1}{2}\left(\mathrm{e}_{x} \otimes \mathrm{e}_{x}-\mathrm{e}_{y} \otimes \mathrm{e}_{y}\right) \pm \frac{1}{2} i\left(\mathrm{e}_{x} \otimes \mathrm{e}_{y}+\mathrm{e}_{y} \otimes \mathrm{e}_{x}\right), \\
& \mathrm{t}^{ \pm 1}=\mp \frac{1}{2}\left(\mathrm{e}_{x} \otimes \mathrm{e}_{z}+\mathrm{e}_{z} \otimes \mathrm{e}_{x}\right)-\frac{1}{2} i\left(\mathrm{e}_{y} \otimes \mathrm{e}_{z}+\mathrm{e}_{z} \otimes \mathrm{e}_{y}\right), \\
& \mathrm{t}^{0}=6^{-1 / 2}\left(-\mathrm{e}_{x} \otimes \mathrm{e}_{x}-\mathrm{e}_{y} \otimes \mathrm{e}_{y}+2 \mathrm{e}_{z} \otimes \mathrm{e}_{z}\right), \\
& 3^{-1 / 2} \delta=3^{-1 / 2}\left(\mathrm{e}_{x} \otimes \mathrm{e}_{x}+\mathrm{e}_{y} \otimes \mathrm{e}_{y}+\mathrm{e}_{z} \otimes \mathrm{e}_{z}\right) .
\end{aligned}
$$

Mathews then couples these basis tensors to the scalar spherical harmonics to obtain the six basis harmonics

$$
\begin{aligned}
& \mathrm{T}^{2 l^{\prime}, l m}=\sum_{m^{\prime}=-l^{\prime}}^{l^{\prime}} \sum_{m^{\prime \prime}=-2}^{2}\left(l^{\prime} 2 m^{\prime} m^{\prime \prime} \mid l m\right) Y^{l^{\prime} m^{\prime}} \mathbf{t}^{m^{\prime \prime}}, \\
& l^{\prime}=l \pm(0,1, \text { or } 2) ; \\
& \mathrm{T}^{0 l, l m}=-Y^{l m} 3^{-1 / 2} \delta .
\end{aligned}
$$

[Mathews (1962) uses the notation $\mathrm{T}_{l l^{\prime} m}$ for $\mathrm{T}^{2 l^{\prime}, l m}$; and actually, he never introduces or uses $\mathrm{T}^{0 l, l m}$; Zerilli (1970) defines the analogous, antisymmetric, $\mathrm{T}^{1 l^{\prime}, l m}$, and he uses the notation $\mathrm{T}_{l l^{\prime} m}^{(\lambda)}$ for our $T^{\lambda l^{\prime}, l m}$.] The pureorbital harmonics $\mathrm{T}^{\lambda l^{\prime}, l m}$ with fixed $\lambda, l^{\prime}$, and $l$ transform among each other under rotations according to an irreducible representation of order $l$. These harmonics are orthornormal

$$
\int T_{j k}^{\lambda l, L M} T_{j k}^{\lambda^{\prime} l^{\prime}, L^{\prime} M^{\prime} *} d \Omega=\delta_{\lambda \lambda^{\prime}} \delta_{l l^{\prime}} \delta_{L L^{\prime}} \delta_{M M^{\prime}} ;
$$

they have parity $\pi=(-1)^{l^{\prime}}$; and under complex conjugation they transform as

$$
\mathrm{T}^{\lambda l^{\prime}, l m *}=(-1)^{l^{\prime}+l+m} \mathrm{~T}^{\lambda l^{\prime}, l-m} \text { for } \lambda=0 \text { or } 2 \text {. }
$$

These pure-orbital tensor harmonics are nicely related to solutions of Laplace's equation (Sec. II.F) and the wave equation (Sec. II.G). However, they are not optimally designed for describing radiation in the radiation zone because, under local rotations about the radial vector, their tensor components (for fixed $\lambda, l^{\prime}, l, m$ ) do not transform as the components of the polarization tensor of a pure spin state. The following "pure-spin tensor harmonics" introduced by Zerilli (1970) [and for spin 2 by Mathews (1962)], do behave like pure-spin states \{note Zerilli's sign error on the second term of Eq. (2.30a)-his Eq. (6c); also note that the fourth line of his Eq. (6a) should read $\times[J(J-1)]^{1 / 2}[(2 J-1)$ $\left.(2 J+1)]^{-1 / 2} \mathrm{~T}_{J, J-2, M}^{(2)}\right\}$ :

$$
\begin{aligned}
\mathrm{T}^{L 0, l m}= & \left(\frac{(l+1)(l+2)}{(2 l+1)(2 l+3)}\right)^{1 / 2} \mathrm{~T}^{2 l+2, l m} \\
& -\left(\frac{2 l(l+1)}{3(2 l-1)(2 l+3)}\right)^{1 / 2} \mathrm{~T}^{2 l, l m} \\
& +\left(\frac{(l-1) l}{(2 l-1)(2 l+1)}\right)^{1 / 2} \mathrm{~T}^{2 l-2, l m}-\frac{1}{3^{1 / 2}} \mathrm{~T}^{0 l, l m} \\
= & \mathrm{n} \otimes \mathrm{n} Y^{l m} ; \\
\mathrm{T}^{T 0, l m}= & -\left(\frac{(l+1)(l+2)}{2(2 l+1)(2 l+3)}\right)^{1 / 2} \mathrm{~T}^{2 l+2, l m} \\
& +\left(\frac{l(l+1)}{3(2 l-1)(2 l+3)}\right)^{1 / 2} \mathrm{~T}^{2 l, l m} \\
& -\left(\frac{(l-1) l}{2(2 l-1)(2 l+1)}\right)^{1 / 2} \mathrm{~T}^{2 l-2, l m}-\left(\frac{2}{3}\right)^{1 / 2} \mathrm{~T}^{0 l, l m} \\
= & 2^{-1 / 2}(\delta-\mathrm{n} \otimes \mathrm{n}) Y^{l m} ;
\end{aligned}
$$

$$
\mathbf{T}^{E 1, l m}=-\left(\frac{2 l(l+2)}{(2 l+1)(2 l+3)}\right)^{1 / 2} \mathbf{T}^{2 l+2, l m}
$$$$
-\left(\frac{3}{(2 l-1)(2 l+3)}\right)^{1 / 2} T^{2 l, l m}
$$$$
+\left(\frac{2(l-1)(l+1)}{(2 l-1)(2 l+1)}\right)^{1 / 2} \mathbf{T}^{2 l-2, l m}
$$$$
=2^{1 / 2}\left[\mathrm{n} \otimes \mathrm{Y}^{E, l m}\right]^{\mathrm{S}}=\left(\frac{2}{l(l+1)}\right)^{1 / 2}\left[\mathrm{n} \otimes r \nabla Y^{l m}\right]^{\mathrm{S}}
$$$$
=\left[-2 \mathbf{n} \times \mathbf{T}^{B 1, l m}\right]^{\mathrm{s}} \text {; }
$$

$$
\mathrm{T}^{E 2, l m}=\left(\frac{l(l-1)}{2(2 l+1)(2 l+3)}\right)^{1 / 2} \mathrm{~T}^{2 l+2, l m}
$$$$
+\left(\frac{3(l-1)(l+2)}{(2 l-1)(2 l+3)}\right)^{1 / 2} \mathrm{~T}^{2 l, l m}
$$$$
+\left(\frac{(l+1)(l+2)}{2(2 l-1)(2 l+1)}\right)^{1 / 2} \mathbf{T}^{2 l-2, l m}
$$$$
=\left(\frac{2}{(l-1)(l+2)}\right)^{1 / 2}\left[r \nabla \mathbf{Y}^{E, l m}\right]^{\mathrm{STT}}
$$$$
=-\left(\frac{2}{(l-1)(l+2)}\right)^{1 / 2}\left[i \mathrm{LY}^{B, l m}\right]^{\mathrm{STT}}
$$$$
=\left(2 \frac{(l-2) !}{(l+2) !}\right)^{1 / 2}\left[r^{2} \nabla \nabla Y^{l m}\right]^{\mathrm{STT}}
$$$$
=\left(2 \frac{(l-2) !}{(l+2) !}\right)^{1 / 2}\left[\operatorname{LLY} Y^{l m}\right]^{\text {ST T }}
$$$$
=\left[\mathbf{- n} \times \mathbf{T}^{B 2, l \mathrm{~m}}\right]^{\mathrm{s}} \text {; }
$$

$$
\begin{aligned}
\mathbf{T}^{B 1, l m} & =i\left(\frac{l+2}{2 l+1}\right)^{1 / 2} \mathbf{T}^{2 l+1, l m}-i\left(\frac{l-1}{2 l+1}\right)^{1 / 2} \mathbf{T}^{2 l-1, l m} \\
& =2^{1 / 2}\left[\mathbf{n} \otimes \mathbf{Y}^{B, l m}\right]^{\mathrm{S}}=\left(\frac{2}{l(l+1)}\right)^{1 / 2}\left[\mathbf{n} \otimes i \mathbf{L} Y^{l m}\right]^{\mathrm{S}} \\
& =\left[2 \mathrm{n} \times \mathbf{T}^{E 1, l m}\right]^{\mathrm{S}}
\end{aligned}
$$




$$
\begin{aligned}
\mathbf{T}^{B 2, l m} & =-i\left(\frac{l-1}{2 l+1}\right)^{1 / 2} \mathbf{T}^{2 l+1, l m}-i\left(\frac{l+2}{2 l+1}\right)^{1 / 2} \mathbf{T}^{2 l-1, l m} \\
& =\left(\frac{2}{(l-1)(l+2)}\right)^{1 / 2}\left[i \mathrm{~L} \mathrm{Y}^{E, l m}\right]^{\mathrm{STT}} \\
& =\left(\frac{2}{(l-1)(l+2)}\right)^{1 / 2}\left[r \nabla \mathrm{Y}^{B, l m}\right]^{\mathrm{STT}} \\
& =\left(2 \frac{(l-2) !}{(l+2) !}\right)^{1 / 2}\left[i \mathrm{~L} r \nabla Y^{l m}\right]^{\mathrm{STT}} \\
& =\left(2 \frac{(l-2) !}{(l+2) !}\right)^{1 / 2}\left[i r \nabla L Y^{l m}\right]^{\mathrm{STT}} \\
& =\left[\mathbf{n} \times \mathbf{T}^{E 2, l m}\right]^{\mathrm{S}} .
\end{aligned}
$$

Here a superscript S means "symmetric part of" and superscript TT means "transverse traceless part of" [Eqs. (1.7b) and (1.11)]; $\nabla$ is the gradient operator and $\mathrm{L}$ is the angular momentum operator [Eq. (2.19)]. [Zerilli (1970) uses the notation

$$
\begin{aligned}
& \mathbf{a}_{l m}=\mathbf{T}^{L 0, l m}, \quad \mathbf{b}_{l m}=\mathbf{T}^{E 1, l m}, \quad \mathbf{c}_{l m}=-i \mathbf{T}^{B 1, l m}, \\
& \mathbf{d}_{l m}=-i \mathbf{T}^{B 2, l m}, \quad \mathbf{f}_{l m}=\mathbf{T}^{E 2, l m}, \quad \mathbf{g}_{l m}=\mathbf{T}^{T 0, l m} ;
\end{aligned}
$$

and Mathews (1962) uses the notation

$$
\mathbf{T}_{l m}^{m}=\mathbf{T}^{E 2, l m}, \quad \mathbf{T}_{l m}^{e}=i \mathbf{T}^{B 2, l m} .
$$

Zerilli and Mathews, and also Wagoner (1977) use the terms "electric-type parity" and "magnetic-type parity" to refer to $\pi=(-1)^{l+1}$ and $\pi=(-1)^{l}$, respectively-which is opposite to our terminology and to that found every where else in the general relativity literature. Their terminology leads to the objectionable convention that the gravitational waves produced by a slow-motion source are predominantly of "magnetic quadrupole" type; our terminology makes them "electric quadrupole."] The transformation $(2.30)$ from pure-orbital to pure-spin harmonics is unitary; therefore it is easily inverted:

$$
\begin{aligned}
\mathbf{T}^{0 l, l m}= & -3^{-1 / 2} \mathbf{T}^{L 0, l m}-\left(\frac{2}{3}\right)^{1 / 2} \mathbf{T}^{T 0, l m}, \\
\mathbf{T}^{2 l-2, l m}= & \left(\frac{(l-1) l}{(2 l-1)(2 l+1)}\right)^{1 / 2} \mathbf{T}^{L 0, l m} \\
& -\left(\frac{(l-1) l}{2(2 l-1)(2 l+1)}\right)^{1 / 2} \mathbf{T}^{T 0, l m} \\
& +\left(\frac{2(l-1)(l+1)}{(2 l-1)(2 l+1)}\right)^{1 / 2} \mathbf{T}^{E 1, l m} \\
& +\left(\frac{(l+1)(l+2)}{2(2 l-1)(2 l+1)}\right)^{1 / 2} \mathbf{T}^{E 2, l m}, \\
\mathbf{T}^{2 l-1, l m}= & i\left(\frac{l-1}{2 l+1}\right)^{1 / 2} \mathbf{T}^{B 1, l m}+i\left(\frac{l+2}{2 l+1}\right)^{1 / 2} \mathbf{T}^{B 2, l m}, \\
\mathbf{T}^{2 l, l m}= & -\left(\frac{2 l(l+1)}{3(2 l-1)(2 l+3)}\right)^{1 / 2} \mathbf{T}^{L 0, l m} \\
& +\left(\frac{l(l+1)}{3(2 l-1)(2 l+3)}\right)^{1 / 2} \mathbf{T}^{T 0, l m} \\
& -\left(\frac{3}{(2 l-1)(2 l+3)}\right)^{1 / 2} \mathbf{T}^{E 1, l m} \\
& +\left(\frac{3(l-1)(l+2)}{(2 l-1)(2 l+3)}\right)^{1 / 2} \mathbf{T}^{E 2, l m},
\end{aligned}
$$

$$
\begin{aligned}
\mathbf{T}^{2 l+1, l m}= & -i\left(\frac{l+2}{2 l+1}\right)^{1 / 2} \mathbf{T}^{B 1, l m}+i\left(\frac{l-1}{2 l+1}\right)^{1 / 2} \mathbf{T}^{B 2, l m}, \\
\mathbf{T}^{2 l+2, l m}= & \left(\frac{(l+1)(l+2)}{(2 l+1)(2 l+3)}\right)^{1 / 2} \mathbf{T}^{L 0, l m} \\
& -\left(\frac{(l+1)(l+2)}{2(2 l+1)(2 l+3)}\right)^{1 / 2} \mathbf{T}^{T 0, l m} \\
& -\left(\frac{2 l(l+2)}{(2 l+1)(2 l+3)}\right)^{1 / 2} \mathbf{T}^{E 1, l m} \\
& +\left(\frac{l(l-1)}{2(2 l+1)(2 l+3)}\right)^{1 / 2} \mathbf{T}^{E 2, l m}
\end{aligned}
$$

For fixed $l, m, \theta, \phi$ the pure-spin harmonics have the following algebraic and directionality properties:

$$
\begin{aligned}
& \mathrm{T}^{L 0, l m}: \text { pure "longitudinal" (i.e., pure radial); } \\
& \mathrm{T}^{T 0, l m}: \text { pure transverse; proportional to the } \\
& \text { transverse projection tensor } P_{j k} ;
\end{aligned}
$$

These algebraic and directionality properties are the same as those of the following pure-spin (i.e., purehelicity) states of radially propagating gravitational waves in general metric theories of gravity [Eardley, Lee, and Lightman (1973); Eardley et al. (1973)], which in turn correspond to the following NewmanPenrose (1962) tetrad components of the Riemann tensor of a wave

$$
\begin{aligned}
& \mathbf{T}^{L 0, l m}: \text { longitudinal, spin } 0\left(\text { Newman-Penrose } \Psi_{2}\right), \\
& \left.T^{T 0, l m}: \text { transverse, spin } 0 \text { (Newman-Penrose } \Phi_{22}\right), \\
& \left.T^{E 1, l m} \text { and } T^{B 1, l m}: \text { spin } 1 \text { (Newman-Penrose } \Psi_{3}\right), \\
& T^{E 2, l m} \text { and } \mathbf{T}^{B 2, l m}: \text { spin } 2\left(\text { Newman-Penrose } \Psi_{4}\right) .
\end{aligned}
$$

The harmonics $\mathbf{T}^{L 0, l m}, \mathbf{T}^{T 0, l m}, \mathbf{T}^{E 1, l m}$, and $\mathbf{T}^{E 2, l m}$ have "electric-type" parity $\pi=(-1)^{l}$; while $\mathbf{T}^{B 1, l m}$ and $\mathbf{T}^{B 2, l m}$ have "magnetic-type" parity $\pi=(-1)^{l+1}$. The pure-spin harmonics are orthonormal

$$
\int T_{j k}^{J S, l m} T_{j k}^{J^{\prime} S^{\prime}, l^{\prime} m^{\prime} *} d \Omega=\delta_{J J}, \delta_{S S^{\prime}} \delta_{l l l^{\prime}} \delta_{m m^{\prime}} ;
$$

and their complex conjugates are given by

$$
\mathbf{T}^{J S, l m *}=(-1)^{m} \mathbf{T}^{J S, l m} \text {. }
$$

[The factor $i$ was included in Eqs. (2.30e) and (2.30f) in order to produce this complex-conjugate relation.] These pure-spin tensor harmonics are intimately related to the Regge-Wheeler (1957) tensor harmonics [see Zerilli (1969, 1970), Sandberg (1978)]:

$$
\begin{aligned}
& \mathbf{T}^{L 0, l m}=Y^{l m} \boldsymbol{\delta}-\boldsymbol{\Phi}^{l m}, \\
& \mathbf{T}^{T 0, l m}=2^{-1 / 2} \boldsymbol{\Phi}^{l m}, \\
& \mathbf{T}^{E 1, l m}=\left(\frac{2}{l(l+1)}\right)^{1 / 2}\left[\mathbf{n} \otimes \boldsymbol{\Psi}^{l m}\right]^{\mathrm{s}}, \\
& \mathbf{T}^{B 1, l m}=\left(\frac{2}{l(l+1)}\right)^{1 / 2}\left[\mathbf{n} \otimes \Phi^{l m}\right]^{\mathrm{s}},
\end{aligned}
$$




$$
\begin{aligned}
\mathbf{T}^{E 2, l m} & =\left(2 \frac{(l-2) !}{(l+2) !}\right)^{1 / 2}\left(\Psi^{l m}+\frac{l(l+1)}{2} \Phi^{l m}\right) \\
& =\left(2 \frac{(l-2) !}{(l+2) !}\right)^{1 / 2}\left[\Psi^{l m}\right]^{\mathbf{T} \mathbf{T}}, \\
\mathbf{T}^{B 2, l m} & =\left(2 \frac{(l-2) !}{(l+2) !}\right)^{1 / 2} \chi^{l m},
\end{aligned}
$$

and also to the Newman-Penrose (1966) spin-weighted spherical harmonics [for details of which see Goldberg et al. (1967)]:

$$
\begin{aligned}
& \mathbf{T}^{L 0, l m}={ }_{0} Y^{l m} \mathbf{n} \otimes \mathbf{n}, \\
& \mathbf{T}^{T 0, l m}=2^{-1 / 2}{ }_{0} Y^{l m}(\boldsymbol{\delta}-\mathbf{n} \otimes \mathbf{n}), \\
& \mathbf{T}^{E 1, l m}=\left[{ }_{-1} Y^{l m} \mathbf{m} \otimes \mathbf{n}-{ }_{1} Y^{l m} \mathbf{m}^{*} \otimes \mathbf{n}\right]^{\mathrm{S}}, \\
& \mathbf{T}^{B 1, l m}=-i\left[{ }_{-1} Y^{l m} \mathbf{m} \otimes \mathbf{n}+{ }_{1} Y^{l m} \mathbf{m}^{*} \otimes \mathbf{n}\right]^{\mathrm{S}}, \\
& \mathbf{T}^{E 2, l m}=2^{-1 / 2}\left[{ }_{-2} Y^{l m} \mathbf{m} \otimes \mathbf{m}+{ }_{2} Y^{l m} \mathbf{m}^{*} \otimes \mathbf{m}^{*}\right], \\
& \mathbf{T}^{B 2, l m}=-i 2^{-1 / 2}\left[{ }_{-2} Y^{l m} \mathbf{m} \otimes \mathbf{m}-{ }_{2} Y^{l m} \mathbf{m}^{*} \otimes \mathbf{m}^{*}\right]
\end{aligned}
$$

The STF version of tensor spherical harmonics can be obtained by inserting expression (2.11) for $Y^{l m}$ into Eqs. (2.30) for the pure-spin harmonics:

$$
\begin{aligned}
T_{j k}^{L 0, l m}= & n_{j} n_{k} \mathcal{Y}_{A_{l}}^{l m} N_{A_{l}}, \\
T_{j k}^{T 0, l m}= & 2^{-1 / 2}\left(\delta_{j k}-n_{j} n_{k}\right) \mathcal{Y}_{A_{l}}^{l m} N_{A_{l}}, \\
T_{j k}^{E 1, l m}= & {[2 l /(l+1)]^{1 / 2} } \\
& \times\left[n_{(j} \mathcal{Y}_{k) A_{l-1}^{l m}}^{l m} N_{A_{l-1}}-n_{j} n_{k} \mathcal{Y}_{A_{l}}^{l m} N_{A_{l}}\right], \\
T_{j k}^{B 1, l m}= & {[2 l /(l+1)]^{1 / 2}\left[n_{(j} \epsilon_{k) p q} n_{p} \mathcal{Y}_{q A_{l-1}}^{l m} N_{A_{l-1}}\right], } \\
T_{j k}^{E 2, l m}= & \left(\frac{2(l-1) l}{(l+1)(l+2)}\right)^{1 / 2}\left[\mathcal{Y}_{j k A_{l-2}}^{l m} N_{A_{l-2}}\right]^{\mathrm{TT}}, \\
T_{j k}^{B 2, l m}= & \left(\frac{2(l-1) l}{(l+1)(l+2)}\right)^{1 / 2}\left[n_{p} \epsilon_{p q(j} \mathcal{Y}_{k) q A_{l-2}}^{l m} N_{A_{l-2}}\right]^{\mathrm{TT}} .
\end{aligned}
$$

By comparing with Eqs. (2.33) one can derive the relation between the pure-orbital harmonics and the STF harmonics:

$$
\begin{aligned}
& T_{j k}^{0}{ }_{j k}^{l, l m}=\frac{(-1)^{l+1}}{3^{1 / 2}(2 l-1) ! !} r^{l+1}\left(r^{-1}\right),{ }_{A_{l}} \mathcal{Y}_{A_{l}}^{l m} \delta_{j k}=\frac{-1}{3^{1 / 2}} \mathcal{Y}_{A_{l}}^{l m} N_{A_{l}} \delta_{j k} \\
& T_{j k}^{2 l, l m}=\frac{(-1)^{l+1}}{(2 l-1) ! !}\left(\frac{6 l(2 l-1)}{(l+1)(2 l+3)}\right)^{1 / 2} r^{l+1}\left[\left(r^{-1}\right),{ }_{A_{l-1}(j} \mathcal{Y}_{k) A_{l-1}}^{l m}-\frac{1}{3}\left(r^{-1}\right),{ }_{A_{l}} \mathcal{Y}_{A_{l}}^{l m} \delta_{j k}\right] \\
& =-\left(\frac{6 l(2 l-1)}{(l+1)(2 l+3)}\right)^{1 / 2}\left[n_{(j} \mathcal{Y}_{k) A_{l-1}^{l m}}^{l m} N_{A_{l-1}}-\left(\frac{l-1}{2 l-1}\right) \mathcal{Y}_{j k A_{l-2}}^{l m} N_{A_{l-2}}-\frac{1}{3} \mathcal{Y}_{A_{l}}^{l m} N_{A_{l}} \delta_{j k}\right] \text {, } \\
& T_{j k}^{2 l-2, l m}=\frac{(-1)^{l}(2 l-3)}{(2 l-3) ! !}\left(\frac{(l-1) l}{(2 l-1)(2 l+1)}\right)^{1 / 2} r^{l-1}\left(r^{-1}\right)_{, A_{l-2}} \mathcal{Y}_{j k A}^{l m}{ }_{l-2}=\left(\frac{(l-1) l}{(2 l-1)(2 l+1)}\right)^{1 / 2} \mathcal{Y}_{j k A_{l-2}}^{l m} N_{A_{l-2}} \text {, } \\
& T_{j k}^{2 l+2, l m}=\frac{(-1)^{l}}{(2 l+3) ! !}\left(\frac{(2 l+1)(2 l+3)}{(l+1)(l+2)}\right)^{1 / 2} r^{l+3}\left(r^{-1}\right), A_{l} l_{k} \mathcal{Y}_{A_{l}}^{l m}=\left(\frac{(2 l+1)(2 l+3)}{(l+1)(l+2)}\right)^{1 / 2} \\
& \times\left[\mathcal{Y}_{A_{l}}^{l m} N_{A_{l}} n_{j} n_{k}-\left(\frac{2 l}{2 l+3}\right) n_{(j} \mathcal{Y}_{k) A_{l-1}}^{l m} N_{A_{l-1}}+\frac{l(l-1)}{(2 l+1)(2 l+3)} \mathcal{Y}_{j k A_{l-2}}^{l m} N_{A_{l-2}}-\frac{1}{2 l+3} \mathcal{Y}_{A_{l}}^{l m} N_{A_{l}} \delta_{j k}\right], \\
& T_{j k}^{2 l-1, l m}=i \frac{(-1)^{l-1}(2 l-1)}{(2 l-1) ! !}\left(\frac{2 l(l-1)}{(l+1)(2 l+1)}\right)^{1 / 2} r^{l}\left(r^{-1}\right)_{, A_{l-2} p} \epsilon_{p q(j} \mathcal{Y}_{k) q A_{l-2}}^{l m}=i\left(\frac{2 l(l-1)}{(l+1)(2 l+1)}\right)^{1 / 2} \epsilon_{p q(j} \mathcal{Y}_{k) q A_{l-2}}^{l m} n_{p} N_{A_{l-2}}, \\
& T_{j k}^{2 l+1, l m}=i \frac{(-1)^{l}}{(2 l+1) ! !}\left(\frac{2 l(2 l+1)}{(l+1)(l+2)}\right)^{1 / 2} r^{l+2}\left(r^{-1}\right)_{, A_{l-1} p(j} \epsilon_{k) p q} \mathcal{Y}_{q A}^{l m}{ }_{l-1} \\
& =-i\left(\frac{2 l(2 l+1)}{(l+1)(l+2)}\right)^{1 / 2}\left[n_{(j} \epsilon_{k) p q} n_{p} \mathcal{Y}_{q A_{l-1}}^{l m} N_{A_{l-1}}-\left(\frac{l-1}{2 l+1}\right) \epsilon_{p q(j} \mathcal{Y}_{k) q A}^{l m} n_{l-2} N_{A_{l-2}}\right]
\end{aligned}
$$

An arbitrary tensor field $\mathbf{w}(\theta, \phi)$ can be expanded in terms of pure-orbital harmonics or pure-spin harmonics or Regge-Wheeler harmonics or spin-weighted harmonics; and one can read off the relationships between the expansion coefficients by examining the relationships between the harmonics themselves and by invoking Eqs. (2.13). The situation is fully analogous to the vector case [Eqs. (2.25)].

\section{F. Solutions of Laplace's equation}

When solving Laplace's equation or the flat-space wave equation it is most convenient to use sets of spherical harmonics which are eigenfunctions of the "orbital angular momentum" operator

$$
\mathbf{L}^{2}=-r^{2} \nabla^{2}+\partial_{r} r^{2} \partial_{r}
$$

The pure-orbital harmonics are eigenfunctions of $\mathrm{L}^{2}$,

$$
\begin{aligned}
& \mathbf{L}^{2} Y^{l m}=l(l+1) Y^{l m}, \\
& \mathbf{L}^{2} \mathbf{Y}^{l^{\prime}, l m}=l^{\prime}\left(l^{\prime}+1\right) \mathbf{Y}^{l^{\prime}, l m}, \\
& \mathbf{L}^{2} \mathbf{T}^{\lambda l^{\prime}, l m}=l^{\prime}\left(l^{\prime}+1\right) \mathrm{T}^{\lambda l^{\prime}, l m} ;
\end{aligned}
$$

but the pure-spin harmonics, the Regge-Wheeler harmonics, and the spin-weighted harmonics are not.

By virtue of Eqs. (2.42), the general scalar, vector, and tensor solutions of Laplace's equation $\nabla^{2} \psi=0$ are

$$
\begin{aligned}
& F(r, \theta, \phi)=\sum_{l, m}\left(F^{l m} \gamma^{-(l+1)}+G^{l m} \gamma^{l}\right) Y^{l m}, \\
& \mathrm{~V}(r, \theta, \phi)=\sum_{l^{i}, l, m}\left(F^{l}, l m \gamma^{-\left(l^{\prime}+1\right)}+G^{l^{l}, l m} \gamma^{l^{\prime}}\right) \mathbf{Y}^{l^{\prime}, l m},
\end{aligned}
$$




$$
\mathrm{U}(r, \theta, \phi)=\sum_{\lambda, l^{\prime}, l, m}\left(F^{\lambda l^{\prime}, l m} V^{-\left(l^{\prime}+1\right)}+G^{\lambda l^{\prime}, l m} \gamma^{l^{\prime}}\right) \mathrm{T}^{\lambda l^{\prime}, l m} .
$$

One can construct the STF form of these solutions by inserting expressions (2.11), (2.24), and (2.40) into Eqs. (2.43), by using the relation $r_{, j}=n_{j}$, by using the symmetry and trace-free features of $\mathcal{Y}_{A_{l}}^{l m}$, and by making suitable changes of notation. The result for the general solution that is well behaved at infinity is

$$
\begin{aligned}
& F(r, \mathrm{n})=\sum_{l=0}^{\infty} a_{A_{l}}\left(\frac{1}{r}\right)_{, A_{l}}, \\
& V_{j}(r, \mathbf{n})=\sum_{l=1}^{\infty}\left[\AA_{j A_{l-1}}\left(\frac{1}{r}\right)_{, A_{l-1}}+\epsilon_{j p q} \mathbf{e}_{q A_{l-1}}\left(\frac{1}{r}\right)_{, p A_{l-1}}\right] \\
& +\sum_{l=0}^{\infty} D_{A_{l}}\left(\frac{1}{r}\right)_{, j A_{l}}, \\
& U_{j k}(r, \mathrm{n})=\sum_{l=0}^{\infty} \delta_{j k} \mathcal{E}_{A_{l}}\left(\frac{1}{r}\right)_{, A_{l}} \\
& +\sum_{l=2}^{\infty}\left[\Im_{j k A_{l-2}}\left(\frac{1}{r}\right)_{, A_{l-2}}+\epsilon_{p q j} \mathfrak{S}_{k q A_{l-2}}\left(\frac{1}{r}\right)_{, p A_{l-2}}\right]^{S} \\
& +\sum_{l=1}^{\infty}\left[\mathfrak{C C}_{j A_{l-1}}\left(\frac{1}{r}\right)_{, k A}+\epsilon_{j p q} \boldsymbol{g}_{q A_{l-1}}\left(\frac{1}{r}\right)_{, k p A_{l-1}}\right]^{S} \\
& +\sum_{l=0}^{\infty} \partial_{A_{l}}\left(\frac{1}{r}\right)_{, j k A_{l}} \text {. }
\end{aligned}
$$

The solutions which blow up at infinity are less simply expressed in STF form. It is easy to see from $\nabla^{2}(1 / r)$ $=-4 \pi \delta_{3}(\mathrm{x})$ that the STF expressions (2.44) do satisfy Laplace's equation everywhere except at the singular point $r=0$.

\section{G. Solutions of the wave equation}

It is useful to introduce the following "basis" solutions to the vacuum wave equation $\square \psi=\left(-\partial_{t}^{2}+\nabla^{2}\right) \psi=0$ :

$$
\begin{aligned}
\Phi^{\epsilon \omega l m}(t, r, \theta, \phi)= & (|\omega| / 2 \pi)^{1 / 2} e^{-i \omega t} h^{\epsilon}(\omega r) Y^{l m}(\theta, \phi), \\
\Phi_{j}{ }^{\epsilon \omega l^{\prime} l m}(t, r, \theta, \phi)= & (|\omega| / 2 \pi)^{1 / 2} e^{-i \omega t} \\
& \times h^{\epsilon l^{\prime}}(\omega r) Y_{j}^{l^{\prime}, l m}(\theta, \phi),
\end{aligned}
$$

$$
\begin{aligned}
\Phi_{j k}^{\epsilon \omega \lambda l^{\prime} l m}(t, r, \theta, \phi)= & (|\omega| / 2 \pi)^{1 / 2} e^{-i \omega t} \\
& \times h^{\epsilon l^{\prime}}(\omega r) T_{j k}^{\lambda l^{\prime}, l m}(\theta, \phi) .
\end{aligned}
$$

Here $\omega$ is a real frequency and $\epsilon$ is either + (outgoing waves) or - (ingoing waves). We use the unconventional notation $h^{\epsilon} l(x)$ for spherical Hankel functions

$$
h^{+l}=h_{l}^{(1)}=j^{l}+i y^{l}, \quad h^{-l}=h_{l}^{(2)}=j^{l}-i y^{l},
$$

where $j^{l}$ and $y^{l}$ are the spherical Bessel functions. Some useful formulas are

$$
\begin{aligned}
h^{\epsilon l}(x)= & -\epsilon i x^{l}\left(-\frac{1}{x} \frac{d}{d x}\right)^{l}\left(\frac{e^{\epsilon i x}}{x}\right), \\
h^{\epsilon l}(x)= & e^{\epsilon i x} \sum_{k=0}^{l} \frac{(-\epsilon i)^{l+1-k}(l+k) !}{2^{k} k !(l-k) !} \frac{1}{x^{k+1}}, \\
h^{\epsilon l}(x)= & \sum_{k=0}^{\infty} \frac{(-1)^{k} x^{l+2 k}}{2^{k} k !(2 l+2 k+1) ! !} \\
& +\epsilon i \sum_{k=0}^{\infty} \frac{(-1)^{k}(2 l+1) ! !}{2^{k} k !(-1-2 l)(1-2 l) \cdots(2 k-1-2 l)} \frac{1}{x^{l+1-2 k}} .
\end{aligned}
$$

In addition to the outgoing and ingoing basis solutions, it is useful to define standing-wave basis solutions

$$
\begin{aligned}
& \Phi^{S \omega l m}=\frac{1}{2}\left(\Phi^{+\omega l m}+\Phi^{-\omega l m}\right), \\
& \Phi_{j}^{S \omega l^{\prime} l m}=\frac{1}{2}\left(\Phi_{j}^{+\omega l^{\prime} l m}+\Phi_{j}^{-\omega l^{\prime} l m}\right), \\
& \Phi_{j k}^{S \omega \lambda l^{\prime} l m}=\frac{1}{2}\left(\Phi_{j k}^{+\omega \lambda l^{\prime} l m}+\Phi_{j k}^{-\omega \lambda l^{\prime} l m}\right) .
\end{aligned}
$$

Note that they are given by expressions (2.45) with $h^{\epsilon} l(\omega r)$ replaced by $j^{l}(\omega r)=\operatorname{Re}\left[h^{\epsilon l}(\omega r)\right]$.

From the basis solutions (2.45) and (2.48) one can construct scalar, vector, and symmetric-tensor Green's functions which satisfy

$$
\begin{aligned}
& \square G^{\epsilon}\left(x, x^{\prime}\right)=-\delta_{4}\left(x-x^{\prime}\right), \\
& \square G_{j \cdot k}^{\epsilon}\left(x, x^{\prime}\right)=-\delta_{j k} \delta_{4}\left(x-x^{\prime}\right), \\
& \square G_{j k \cdot p q}^{\epsilon}\left(x, x^{\prime}\right)=-\frac{1}{2}\left(\delta_{j p} \delta_{k q}+\delta_{j q} \delta_{k p}\right) \delta_{4}\left(x-x^{\prime}\right),
\end{aligned}
$$

and which have outgoing waves at infinity for $\epsilon=+1$, ingoing for $\epsilon=-1$. The Green's functions are given by

$$
\begin{aligned}
G^{\epsilon}\left(x, x^{\prime}\right)= & \epsilon i \sum_{l, m} \int d \omega\left\{\operatorname{sgn}(\omega) \Phi^{\epsilon \omega l m}(x)\left[\Phi^{S \omega l m}\left(x^{\prime}\right)\right]^{*}\right\} \text { if } r>r^{\prime} \\
= & \epsilon i \sum_{l, m} \int d \omega\left\{\operatorname{sgn}(\omega) \Phi^{S \omega l m}(x)\left[\Phi^{(-\epsilon) \omega l m}\left(x^{\prime}\right)\right]^{*}\right\} \text { if } r<r^{\prime} ; \\
G_{j \cdot k}^{\epsilon}\left(x, x^{\prime}\right)= & \epsilon i \sum_{l^{\prime} l m} \int d \omega\left\{\operatorname{sgn}(\omega) \Phi_{j}^{\epsilon \omega l^{\prime} l m}(x)\left[\Phi_{k}^{S \omega l^{\prime} l m}\left(x^{\prime}\right)\right]^{*}\right\} \quad \text { if } r>r^{\prime} \\
= & \epsilon i \sum_{l^{\prime} l m} \int d \omega\left\{\operatorname{sgn}(\omega) \Phi_{j}^{S \omega l^{\prime} l m}(x)\left[\Phi_{k}^{(-\epsilon) \omega l^{\prime} l m}\left(x^{\prime}\right)\right]^{*}\right\} \text { if } r<r^{\prime} ; \\
G_{j k \cdot p q}^{\epsilon}\left(x, x^{\prime}\right) & =\epsilon i \sum_{\lambda l^{\prime} l m} \int d \omega\left\{\operatorname{sgn}(\omega) \Phi_{j k}^{\epsilon \omega \lambda l^{\prime} l m}(x)\left[\Phi_{p q}^{S \omega \lambda l^{\prime} l m}\left(x^{\prime}\right)\right]^{*}\right\} \quad \text { if } r>r^{\prime} \\
& =\epsilon i \sum_{\lambda l^{\prime} l m} \int d \omega\left\{\operatorname{sgn}(\omega) \Phi_{j k}^{S \omega \lambda l^{\prime} l m}(x)\left[\Phi_{p q}^{(-\epsilon) \omega \lambda l^{\prime} l m}\left(x^{\prime}\right)\right]^{*}\right\} \text { if } r<r^{\prime} .
\end{aligned}
$$

Here $\operatorname{sgn}(\omega)$ is the sign of $\omega(+1$ or -1$)$. One can derive these factorized expansions of the Green's functions by the same standard method as Chrzanowski and Misner (1974) use in the Kerr metric; see their Eqs. (2.15)-(2.19).

The basis solutions (2.45), (2.48), and Green's functions (2.50) do not have simple forms when written in STF 
language. However, the general solutions of the vacuum wave equations do have simple forms [Sachs (1961), Pirani (1964)]:

$$
\begin{aligned}
& F(t, r, \theta, \phi)=\sum_{l=0}^{\infty} \sum_{\epsilon}\left[r^{-1} a_{A_{l}}^{\epsilon}(t-\epsilon r)\right]_{A_{l}}, \\
& \left.V_{j}(t, r, \theta, \phi)=\sum_{l=1}^{\infty} \sum_{\epsilon}\left[r^{-1} \mathbb{B}_{j A_{l-1}}^{\epsilon}(t-\epsilon r)\right]_{A_{l-1}}+\left[r^{-1} \epsilon_{j p q} \mathbf{e}_{q A_{l-1}}^{\epsilon}(t-\epsilon r)\right]_{, p A_{l-1}}\right\}+\sum_{l=0}^{\infty} \sum_{\epsilon}\left[r^{-1} \mathbb{D}_{A_{l}}^{\epsilon}(t-\epsilon r)\right]_{j A_{l}}, \\
& U_{j k}(t, r, \theta, \phi)=\sum_{l=0}^{\infty} \sum_{\epsilon} \delta_{j k}\left[r^{-1} \mathcal{E}_{A_{l}}^{\epsilon}(t-\epsilon r)\right], A_{l} \\
& +\sum_{l=2}^{\infty} \sum_{\epsilon}\left\{\left[r^{-1} \Re_{j k A_{l-2}}^{\epsilon}(t-\epsilon r)\right]_{, A_{l-2}}+\left[r^{-1} \epsilon_{p q(j} \mathcal{G}_{k) \alpha A_{l-2}}^{\epsilon}(t-\epsilon r)\right]_{, p A_{l-2}}\right\} \\
& +\sum_{l=1}^{\infty} \sum_{\epsilon}\left\{\left[r^{-1} \mathcal{K}_{A_{l-1}(j}^{\epsilon}(t-\epsilon r)\right]_{, k) A_{l-1}}+\left(\left[r^{-1} \epsilon_{j p q} \partial_{q A_{l-1}}^{\epsilon}(t-\epsilon r)\right]_{, k p A_{l-1}}\right)^{S}\right\}+\sum_{l=0}^{\infty} \sum_{\epsilon}\left[r^{-1} \boldsymbol{K}_{A_{l}}^{\epsilon}(t-\epsilon r)\right]_{, j k A_{l}} .
\end{aligned}
$$

Here the capital script coefficients are arbitrary functions of $t-\epsilon r$. That these expressions satisfy the wave equation follows trivially from the fact that $r^{-1} f(t-\epsilon r)$ satisfies it:

$$
\square\left[r^{-1} f(t-\epsilon r)\right]=0, \text { except at } r=0 .
$$

In manipulating STF solutions of the wave equation, the following relations - which are valid for any STF tensorare useful:

$$
\begin{aligned}
{\left[r^{-1} \Theta_{A_{l}}(t-\epsilon r)\right]_{A_{l}}=} & \sum_{k=0}^{l} \frac{(-1)^{l}(l+k) ! \epsilon^{l-k}}{2^{k k !}(l-k) !} \frac{1}{r^{k+1}}{ }^{(l-k)} \Theta_{A l}(t-\epsilon r) N_{A_{l}} \quad \text { at all } r \\
= & -\sum_{k=0}^{\infty} \frac{(-1)^{l}(2 l+1) ! !}{2^{k} k !(-1-2 l)(1-2 l) \cdots(2 k-1-2 l)} \frac{1}{r^{l+1-2 k}}{ }^{(2 k)}{B_{A} l}(t) N_{A_{l}} \\
& -\epsilon \sum_{k=0}^{\infty} \frac{r^{l+2 k}}{2^{k} l !(2 l+1+2 k) ! !}{ }^{(2 l+1+2 k)} B_{A_{l}}(t) N_{A_{l}} \quad \text { in near zone. }
\end{aligned}
$$

Here a prefix superscript in parentheses means differentiation

$$
{ }^{(n)} \mathbb{B}(u) \equiv\left(\frac{d}{d u}\right)^{n} \mathbb{B}(u) .
$$

Equation (2.53a) is given on p. 299 of Pirani (1964) for the case $\epsilon=+1$ of outgoing waves [except for an error of $\left.(-1)^{l}\right]$; it can be derived by straightforward differentiation. Expression (2.53b) is derived most easily by comparison of $(2.53 \mathrm{a})$ and $(2.53 \mathrm{~b})$ with the corresponding expansions of spherical Bessel functions, Eqs. (2.47).

\section{REGIONS OF SPACETIME AROUND AN ISOLATED SOURCE}

We shall characterize a source of gravitational waves, semiquantitatively, by the following length scales:

$$
\begin{aligned}
L & \equiv \text { “size of source" } \\
& \equiv\left[\begin{array}{l}
\text { radius of region inside which the } \\
\text { stress -energy } T^{\alpha \beta} \text { is contained }
\end{array}\right], \\
2 M & \equiv \text { "gravitational radius of source" } \\
& \equiv\left[\begin{array}{l}
2 \times \text { mass of source in } \\
\text { units where } G=c=1
\end{array}\right], \\
x & \equiv \text { "reduced wavelength of waves" } \\
& \equiv\left[\begin{array}{l}
1 / 2 \pi \times \text { characteristic wavelength } \\
\text { of gravitational waves emitted } \\
\text { by source }
\end{array}\right],
\end{aligned}
$$

Rev. Mod. Phys., Vol. 52, No. 2, Part I, April 1980
$\left.\begin{array}{l}r_{I} \equiv \text { “inner radius of local wave zone" } \\ r_{0} \equiv \text { "outer radius of local wave zone" }\end{array}\right\}$ (see below).

Corresponding to these length scales, we shall divide space around a source into the following regions:

$$
\begin{array}{ll}
\text { Source: } & r \lesssim L, \\
\text { Strong-field region: } & r \lesssim 10 M \text { if } 10 M z L \\
& \begin{aligned}
\text { typically does not exist } \\
\text { if } L \gg 10 M,
\end{aligned} \\
\text { Weak-field near zone: } & L<r, 10 M \ll r, \quad r \ll x, \\
\text { Local wave zone: } & r_{I} \preccurlyeq r \lesssim r_{0} \\
\text { Distant wave zone: } & r_{0} \lesssim r .
\end{array}
$$

The "local wave zone" is the region in which (i) the source's waves are weak, outgoing ripples on a background spacetime, and (ii) the effects of the background curvature on the wave propagation are negligible.

The inner edge of the local wave zone $\left(r_{I}\right)$ is the location at which one or more of the following effects becomes important: (i) the waves cease to be waves and become a near-zone field, i.e., $r$ becomes $\$ \lambda$; (ii) the gravitational pull of the source produces a significant red shift, i.e., $r$ becomes $\sim 2 M=$ (Schwarzschild radius of source); (iii) the background curvature produced by the source distorts the wave fronts and backscatters the waves significantly, i.e., $\left(r^{3} / M\right)^{1 / 2}$ becomes $\leqslant x$; (iv) the outer limits of the source itself are encountered, i.e., $r$ becomes $\leqslant L=$ (size of source). Thus, the inner edge of the local wave zone is given by 
$r_{I}=\alpha \times \max \left\{\chi, 2 M,\left(M \chi^{2}\right)^{1 / 3}, L\right\}$,

$\alpha \equiv\left(\begin{array}{l}\text { some suitable number } \\ \text { large compared to unity }\end{array}\right)$.

The outer edge of the local wave zone $r_{0}$ is the location at which one or more of the following effects becomes important: (i) a significant phase shift has been produced by the " $M / r$ " gravitational field of the source, i.e., $(M / x) \ln \left(r / r_{I}\right)$ is no longer $\ll \pi$; (ii) the background curvature due to nearby masses or due to the external universe perturbs the propagation of the waves, i.e., $r$ is no longer $\ll R_{B}=$ (background radius of curvature). Thus, the outer edge of the local wave zone is given by

$$
\begin{aligned}
& r_{0}=\min \left[r_{I} \exp (\chi / \beta M), R_{B} / \gamma\right], \\
& \beta, \gamma \equiv\left(\begin{array}{l}
\text { some suitable numbers } \\
\text { large compared to unity }
\end{array}\right) .
\end{aligned}
$$

Of course, we require that our large numbers $\alpha, \beta, \gamma$ be adjusted so that the thickness of the local wave zone is very large compared to the reduced wavelength:

$$
r_{0}-r_{I} \gg x \text {. }
$$

Previous work in gravitational-wave theory has not distinguished the local wave zone from the distant wave zone. I think it useful to make this distinction, and to split the theory of gravitational waves into two corresponding parts: Part one deals with the source's generation of the waves, and with their propagation into the local wave zone; thus, it deals with the spacetime region $r<r_{0}$ (all of spacetime except the distant wave zone). Part two deals with the propagation of the waves from the local wave zone out through the distant wave zone to the observer, i.e., with the region $r>r_{I}$ (all of the wave zone). The two parts, wave generation and wave propagation, overlap in the local wave zone; and the two theories can be matched together there.

By making this split one can simplify the (semi)rigorous theory of wave generation. No longer must that theory face logarithmic divergences due to phase shifts produced by the mass of the source, or the energy in the waves; and no longer need one be terribly careful about choosing coordinates that avoid those divergences, a la Bondi, van der Burg, and Metzner (1962). Rather, one can use any naive, asymptotically Minkowskii coordinate system one wishes in the theory of wave generation. Such a coordinate system will serve just fine to get the waves out of the source and in to the local wave zone. One can then leave to wave propagation theory the delicate task of getting the waves out of the local wave zone, through the region of dangerous logarithmic divergences, and on into the observer's detector. Moreover, for almost all realistic situations wave propagation theory can do its job admirably well using the elementary formalism of geometric optics [e.g., exercise 35.15 of Misner, Thorne, and Wheeler (1973) - cited henceforth as "MTW"-or last section of Thorne (1977)].

Throughout this paper we shall confine attention to sources which possess a local wave zone-and we shall call such sources "isolated." It seems likely that every source of gravitational waves in the Universe today is "isolated." However, in the very early Universe the background curvature $1 / R_{B}^{2}$ was so large that sources might not have been isolated.

In complex situations the location of the local wave zone might not be obvious. Consider, for example, a neutron star passing very near a supermassive black hole. The tidal pull of the hole sets the neutron star into oscillation, and the star's oscillations produce gravitational waves [Mashoon (1973); Turner (1977)]. If the hole is large enough, or if the star is far enough from it, there may exist a local wave zone around the star which does not also enclose the entire hole. Of greater interest-because more radiation will be produced-is the case where the star is very near the hole and the hole is small enough $\left(M_{h} \lesssim 100 M_{\odot}\right)$ to produce large-amplitude oscillations, and perhaps even disrupt the star. In this case, before the waves can escape the influence of the star, they get perturbed by the background curvature of the hole. One must then consider the entire star-hole system as the source, and construct a local wave zone that surrounds them both.

\section{MULTIPOLE EXPANSION OF THE RADIATION FIELD}

\section{A. Radiation field itself}

Consider any isolated source of gravitational radiation. Throughout its local wave zone $r_{0}<r<r_{I}$, by virtue of definitions (3.3) and (3.4) of $r_{0}$ and $r_{I}$, we can treat the waves as linearized metric perturbations propagating on a flat background. To characterize this flat background we introduce Minkowskii coordinates $t, x, y, z$ with the source at rest at the origin ("asymptotic rest frame of source"; cf. Chap. 19 of MTW); and we introduce the corresponding spherical coordinates [Eqs. (1.1)]. In this coordinate system the transverse, traceless part of the metric perturbation-which characterizes the radiation completely (Secs. 35.435.7 of MTW) has the form

$$
h_{j k}^{\mathrm{TT}}=r^{-1} A_{j k}(t-r, \theta, \phi) .
$$

Here $A_{j k}$ is a transverse, traceless function which, in the local wave zone, varies rapidly (length scale $x$ ) in the radial direction but slowly in the transverse directions

$$
A_{j k_{q} r} \sim A_{j k} / x \gg A_{j k_{\theta} \theta} \sim A_{j k, \phi} .
$$

[Recall the definition, Eqs. (1.13), of the comma.]

We shall resolve the angular dependence of the wave amplitude $A_{j k}$ into tensor spherical harmonics. The best set of harmonics to use is the pure-spin set since it has well defined transversality and helicity properties [Eqs. (2.34) and (2.35)]. Because $A_{j k}$ is transverse and traceless, it can contain only the TT harmonics $\mathrm{T}^{E 2, \imath m}$ and $\mathrm{T}^{B 2, \imath m}$; hence, the radiation field must have the form [Mathews (1962)]

$$
\begin{aligned}
h_{j k}^{\mathrm{TT}}=\sum_{l=2}^{\infty} \sum_{m=-l}^{l}\left[r^{-1(l)} I^{l m}(t-r) T_{j k}^{E 2, l m}\right. \\
\left.+r^{-1(l)} S^{l m}(t-r) T_{j k}^{B 2, l m}\right] .
\end{aligned}
$$

The expansion coefficients $I^{1 m}(t-r)$ will be called the "mass multipole moments" of the radiation field, and $S^{\prime m}(t-r)$ will be called the "current multipole moments." The quantities ${ }^{(t)} I^{l m}$ and ${ }^{(t)} S^{l m}$ are the $l$ th time 
derivatives of these moments; i.e., we use the notation

$$
{ }^{(t)} G(u) \equiv\left(\frac{d}{d u}\right)^{\prime} G(u) .
$$

The mass moments generate the electric-parity part of the field, $\pi=(-1)^{t}$; the current moments generate the magnetic-parity part. The fact that $h_{j k}^{\mathrm{TT}}$ must be real, together with the complex-conjugate property (2.36b) of the TT harmonics, implies that the mass and current moments must satisfy

$$
I^{1 m *}=(-1)^{m} I^{l-m}, \quad S^{l m *}=(-1)^{m} S^{l-m} .
$$

In comparing this radiation field with the properties of the source (Sec. V and Part Two), STF notation will be useful. In STF notation, the mass and current $l$ pole moments of the radiation are real, STF- $l$ functions of $t-r$ :

$$
\begin{aligned}
& \mathscr{g}_{A_{l}}(t-r)=\frac{l !}{4}\left(\frac{2(l-1) l}{(l+1)(l+2)}\right)^{1 / 2} \sum_{m=-l}^{l} I^{l m}(t-r) \mathcal{Y}_{A^{\prime}}^{l m}, \\
& \mathcal{S}_{A_{l}}(t-r)=-\frac{(l+1) !}{8 l}\left(\frac{2(l-1) l}{(l+1)(l+2)}\right)^{1 / 2} \sum_{m=-l}^{l} S^{l m}(t-r) \mathcal{Y}_{A_{i}}^{l m} .
\end{aligned}
$$

[The $l$-dependent coefficients are chosen to make $g_{A_{l}}$ and $S_{A}$, have simple links to the theory of the source in the slow-motion limit; see Eqs. (5.19) and (5.28), below.] Relations (4.6) can be inverted by comparing with Eqs. (2.13):

$$
\begin{aligned}
& I^{l m}=\frac{16 \pi}{(2 l+1) ! !}\left(\frac{(l+1)(l+2)}{2(l-1) l}\right)^{1 / 2} g_{A_{l}} Y_{A_{l}}^{l m *}, \\
& S^{l m}=-\frac{32 \pi l}{(l+1)(2 l+1) ! !}\left(\frac{(l+1)(l+2)}{2(l-1) l}\right)^{1 / 2} S_{A_{l}} y_{A_{l}}^{l m *} .
\end{aligned}
$$

By comparing Eqs. (4.6), (4.3), (2.39e), and (2.39f) we obtain the STF multipole expansion of the radiation field [Sachs (1961), Pirani (1964)]

$$
\begin{aligned}
h_{j k}^{\mathrm{TT}}= & {\left[\sum_{l=2}^{\infty}\left(\frac{4}{l !}\right) r^{-1(l) g_{j k A}(t-2}(t-r) N_{A_{l-2}}\right.} \\
& \left.+\sum_{l=2}^{\infty}\left(\frac{8 l}{(l+1) !}\right) r^{-1} \epsilon_{p q(j}(l) g_{k) p A}(t-r) n_{q} N_{A_{l-2}}\right]^{\mathrm{TT}} .
\end{aligned}
$$

Note that this is the radiation-zone form of the most general outgoing-wave transverse-traceless solution of the flat-space wave equation $\square h_{j k}^{T T}=0$ [Eqs. (2.51c)]. Note also that the mass-quadrupole part of the field has the familiar form

$$
\left(h_{j k}^{\mathrm{TT}}\right)_{\text {mass quadrupole }}=\left[2 \gamma^{-1} \ddot{g}_{j k}(t-r)\right]^{\mathrm{T} \mathrm{T}}
$$

[Eqs. (25) and (26) of Einstein (1918); Eq. (36.20) of MTW].

Expressions (4.3) and (4.8) for the radiation field contain only multipoles of quadrupole order and higher $(l \geqslant 2)$. This is because for $l=0$ and 1 the TT tensor spherical harmonics $T_{j k}^{E^{2}, l m}$ and $T_{j k}^{B 2, l m}$ are nonexistent. One can verify their nonexistence either from the STF formulas $(2.39 \mathrm{e})$ and $(2.39 \mathrm{f})$, where the Y's must have at least two indices; or from (i) Eq. (2.30d), where $T^{2 l-2, l m}$ obviously does not exist when $l=0$ or 1 and where the other terms in $\mathbf{T}^{E 2, l m}$ vanish for $l=1$, and (ii) the relation $\mathbf{T}^{B 2, l m}=\left[\mathrm{n} \times \mathrm{T}^{E 2, l m}\right]^{\mathrm{S}}[\mathrm{Eq}$. (2.30f)].

If the radiation field is known, one can project out its multipole moments using the following integrals over the sphere of constant $t-r$ :

$$
\begin{aligned}
& { }^{(l)} I^{l m}=r \int h_{j k}^{\mathrm{TT}} T_{j k}^{E 2, l m *} d \Omega, \\
& { }^{(l)} S^{l m}=r \int h_{j k}^{\mathrm{TT} T} T^{B 2, l m *} d \Omega \\
& { }^{l(l) g_{A_{l}}}=\left[\frac{l(l-1)(2 l+1) ! !}{2(l+1)(l+2)} \frac{r}{4 \pi} \int h_{a_{1} a_{2}}^{\mathrm{TT}} n_{a_{3}} \cdots n_{a_{t}} d \Omega\right]^{\mathrm{STF}}, \\
& { }^{(l) S_{A_{l}}}=\left[\frac{(l-1)(2 l+1) ! !}{4(l+2)} \frac{r}{4 \pi} \int \epsilon_{a_{1} j k} n_{j} h_{k a_{2}}^{\mathrm{TT}} n_{a_{3}} \cdots n_{a_{l}} d \Omega\right]_{(4.11 \mathrm{~b})}^{\mathrm{STF}} .
\end{aligned}
$$

See Eqs. (4.3) and (2.36a); also Eqs. (2.39e), (2.39f), and (4.7).

It is trivial to generalize this multipole expansion to any other metric theory of gravity if one knows the "E(2) classification" of the theory [Eardley et al. (1973)]. One need only include in the expansion of

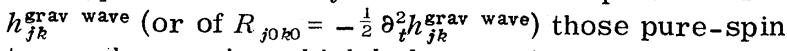
tensor harmonics which belong to the spin states of that $E(2)$ class. For example, in Brans-Dicke theory (class $N_{3}$ ) one must include the harmonics $\mathrm{T}^{T 0,1 m}$ (transverse, spin 0 state), $\mathrm{T}^{E^{2} .}{ }^{1 m}$ (TT, electric-parity, spin 2 state), and $\mathrm{T}^{B 2, l m}$ ( $\mathrm{TT}$, magnetic-parity, spin 2 state).

\section{B. Energy in the waves}

The energy and linear momentum carried off by the radiation field (4.3), (4.8) are most easily evaluated using the Isaacson (1968) stress-energy tensor for gravitational waves

$$
T_{\alpha \beta}^{\mathrm{GW}}=(1 / 32 \pi)\left\langle h_{j k, \alpha}^{\mathrm{TT}} h_{j k_{\beta} \beta}^{\mathrm{TT}}\right\rangle
$$

(cf. MTW, Secs. 35.7 and 35.15). Here the brackets \langle\rangle denote an average over several wavelengths. The power radiated into a unit solid angle about the radial, $n$, direction is

$$
\frac{d E}{d \Omega d t} \equiv-r^{2} n_{j} T_{j 0}^{\mathrm{GW}}=+r^{2} T_{00}^{\mathrm{GW}}
$$

which-by using Eqs. (4.3), (4.8). and (1.11)_works out to be

$$
\begin{aligned}
\frac{d E}{d \Omega d t}=\sum_{l=2}^{\infty} \sum_{l^{\prime}=2}^{\infty} \sum_{m, m^{\prime}} \frac{1}{32 \pi} & \left\langle{ }^{(l+1)} I^{l m\left(l^{\prime}+1\right)} I^{l^{\prime} m^{\prime}} T_{j k}^{E 2, l m} T_{j k}^{E 2,} l^{\prime} m^{\prime}\right. \\
& \left.+{ }^{(l+1)} S^{l m\left(l^{\prime}+1\right)} S^{l^{\prime} m^{\prime}} T_{j k}^{B 2, l m} T_{j k}^{B 2,} l^{\prime} m^{\prime}+2^{(l+1)} I^{l m\left(l^{\prime}+1\right)} S^{l^{\prime} m^{\prime}} T_{j k}^{E 2, l m} T_{j k}^{B 2,} l^{\prime} m^{\prime}\right\rangle,
\end{aligned}
$$




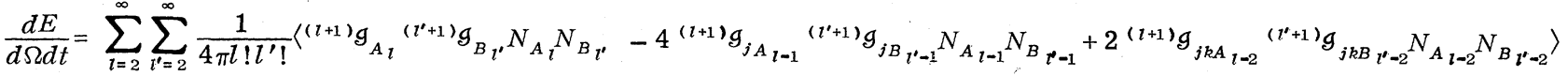

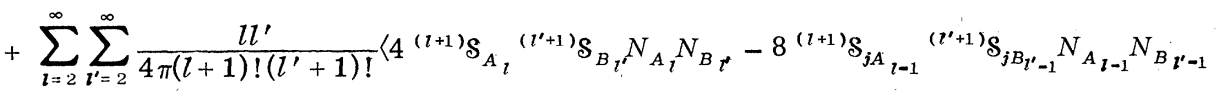

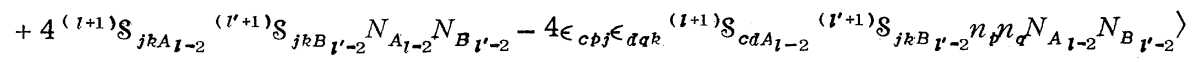

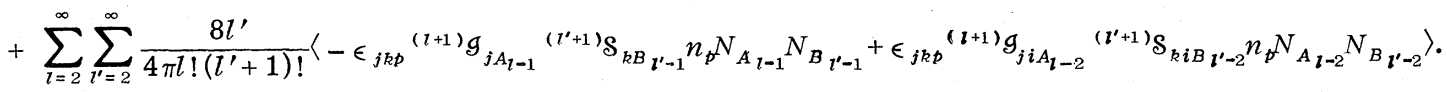

The total power radiated is the integral of this energy flux over a sphere lying in the local wave zone:

$$
\frac{d E}{d t}=\int \frac{d E}{d \Omega d t} d \Omega \text {. }
$$

Expression (4.14) is easily integrated using the orthonormality of the pure-spin tensor harmonics [Eq. (2.36a)] and the complex-conjugate behavior (2.36b), (4.5):

$$
\frac{d E}{d t}=\frac{1}{32 \pi} \sum_{l=2}^{\infty} \sum_{m=-l}^{l}\left\langle\left|{ }^{(l+1)} I^{l m}\right|^{2}+\left|{ }^{(l+1)} S^{l m}\right|^{2}\right\rangle
$$

This result is due to Mathews (1962) [beware, however, his peculiar notation, e.g., $\left.g_{\mu \nu}=\eta_{\mu \nu}+(32 \pi G)^{1 / 2} h_{\mu \nu}\right]$. The analogous STF expression can be found either by inserting Eq. (4.14') into Eq. (4.15) and integrating with the help of Eqs. (2.5) and (2.6); or by taking the claimed answer (4.16') and transforming back to Eq. (4.16) with the help of Eqs. (4.6) and (2.26a). The result is

$$
\begin{aligned}
\frac{d E}{d t}= & \sum_{l=2}^{\infty} \frac{(l+1)(l+2)}{(l-1) l} \frac{1}{l !(2 l+1) ! !}\left\langle(l+1) g_{A_{l}}(l+1) g_{A_{l}}\right\rangle \\
& +\sum_{l=2}^{\infty} \frac{4 l(l+2)}{(l-1)} \frac{1}{(l+1) !(2 l+1) ! !}\left\langle(l+1) \mathrm{S}_{A_{l}}{ }^{(l+1)} \mathrm{S}_{A_{l}}\right\rangle .
\end{aligned}
$$

The mass quadrupole part agrees with Einstein's (1918) formula (30), after correction of his factor -2 error ${ }^{1}$ :

$$
\frac{d E}{d t} \text { mass quadrupole }=\frac{1}{5}\left\langle\dddot{g}_{j k} \dddot{g}_{j k}\right\rangle \text {; }
$$

[cf. Eq. (36.23) of MTW]. The mass octupole and current quadrupole parts agree with Papapetrou's (1962, 1971) formulas -after one invokes Eqs. (5.19) below and changes notation.

\section{Linear momentum in the waves}

The waves from our source carry linear momentum out radially; and, as with any locally plane-fronted radiation field, the magnitude of their momentum flux is the same as that of their energy flux

$$
\frac{d P_{j}}{d \Omega d t} \equiv r^{2} n_{k} T_{k j}^{G W}=r^{2} n_{j} \frac{d E}{d \Omega d t}
$$

[cf. Eq. (35.77j) of MTW]. The total rate at which the source feeds momentum into the radiation field

$$
\frac{d P_{j}}{d t}=\int \frac{d P_{j}}{d \Omega d t} d \Omega
$$

can be calculated by inserting (4.14) into (4.18) and (4.19) and then using group-theoretic methods (Racah recoupling, etc.) to bring the integrand into doable form; the result is

$$
\begin{aligned}
& \frac{d P_{j}}{d t}=\sum_{l=2}^{\infty} \sum_{m} \frac{1}{32 \pi(l+1)}\left(\frac{2(l-1)(l+3)}{(2 l+1)(2 l+3)}\right)^{1 / 2}\left\langle{ }^{(l+1)} I^{l m *\left\{[(l-m+1)(l-m+2)]^{1 / 2(l+2)} I^{l+1} m^{-1} \xi_{j}^{-1}\right.}\right. \\
& \left.\left.+[2(l-m+1)(l+m+1)]^{1 / 2(l+2)} I^{l+1} m \xi_{j}^{0}+[(l+m+1)(l+m+2)]^{1 / 2(l+2)} I^{l+1 m+1} \xi_{j}^{1}\right\}\right\rangle \\
& +\sum_{l=2}^{\infty} \sum_{m} \frac{1}{32 \pi(l+1)}\left(\frac{2(l-1)(l+3)}{(2 l+1)(2 l+3)}\right)^{1 / 2}\left\langle{ }^{(l+1)} S^{l m *\left\{[(l-m+1)(l-m+2)]^{1 / 2(l+2)} S^{l+1} m^{m-1} \xi_{j}^{-1}\right.}\right. \\
& \left.\left.+[2(l-m+1)(l+m+1)]^{1 / 2(l+2)} S^{l+1} m \xi_{j}^{0}+[(l+m+1)(l+m+2)]^{1 / 2(l+2)} S^{l+1} m^{+1} \xi_{j}^{1}\right\}\right\rangle
\end{aligned}
$$

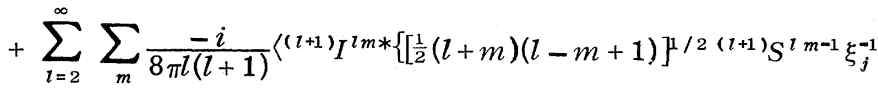

$$
\begin{aligned}
& \left.\left.+m^{(l+1)} S^{l m} \xi_{j}^{0}-\left[\frac{1}{2}(l-m)(l+m+1)\right]^{1 / 2(l+1)} S^{l m+1} \xi_{j}^{1}\right\}\right\rangle .
\end{aligned}
$$

Alternatively, one can insert Eq. (4.14') into Eqs. (4.18) and (4.19), and then integrate with the help of Eqs. (2.5) and (2.6). The result is

$$
\begin{aligned}
& \frac{d P_{j}}{d t}=\sum_{l=2}^{\infty}\left\{\frac{2(l+2)(l+3)}{l(l+1) !(2 l+3) ! !}\left\langle{ }^{(l+2)} g_{j A_{l}}{ }^{(l+1)} g_{A_{l}}\right\rangle+\frac{8(l+3)}{(l+1) !(2 l+3) ! !}\left\langle{ }^{(l+2)} \mathcal{S}_{j A_{l}}{ }^{(l+1)} \mathcal{S}_{A_{l}}\right\rangle\right.
\end{aligned}
$$

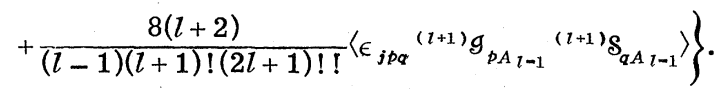

\footnotetext{
1) Einstein (1918) makes a factor 2 error in going from Eq. (9) to (16); and, as a result, his Eqs. (27) and (30) for the energy in the waves are a factor 2 too small.
} 
Once one has derived either Eq. (4.20) or (4.20'), then one can obtain the other from it with the aid of Eqs. (4.6), (4.7), and (2.26). A general expression for $d P_{j} / d t$, presumably equivalent to these but in rather different notation, was derived by Campbell and Morgan (1971). Papapetrou (1962, 1971) derived the leading terms (those involving mass quadrupole, current quadrupole, and mass octupole)_but also in rather different notation.

\section{Angular momentum in the waves}

The dependence of the wave field $h_{j k}^{\mathrm{TT}}$ on angle causes its wave fronts to be not quite precisely spherical--and thereby enables the waves to carry off angular momentum. One might hope that the angular momentum loss could be calculated by integrating $\epsilon_{j a b} x_{a} T_{b 0}^{\mathrm{GW}}$ over a sphere surrounding the source. Unfortunately, such a procedure fails - for this reason: The averaging process that underlies Eq. (4.12) for $T_{\alpha \beta}^{\mathrm{GW}}$ treats as zero the "tiny corrections" which die out as $1 / r^{3}$. However, it is precisely the $1 / r^{3}$ part that carries off the angular momentum. I was vaguely aware of this fact when writing the relevant sections of MTW, but was not sufficiently certain to spell it out explicitly. Subsequently Bryce DeWitt (1971) derived a simple, correct expression for the flux of angular momentum. An alternative derivation, which I have carried out as a check, begins with the fact [MTW Eq. (20.26)] that

$$
\begin{aligned}
\frac{d S_{j}}{d t} & =-\frac{d S_{j}^{\text {source }}}{d t} \equiv-\frac{1}{2} \epsilon_{j a b} \frac{d J_{a b}^{\text {source }}}{d t} \\
& =\int \epsilon_{j a b} x_{a} t_{b c}^{L L} n_{c} r^{2} d \Omega .
\end{aligned}
$$

Here $t_{\alpha \beta}^{L L}$ is the Landau-Lifshitz pseudotensor and $S_{j}^{\text {source }}$ is the intrinsic angular momentum of the source. By expressing $t_{b c}^{L L}$ in terms of the metric perturbation, and by then averaging $\epsilon_{j a} x_{a} t_{b c}^{L L} n_{c}$ over several wavelengths, one ultimately winds up with the simple DeWitt formula

$\frac{d S_{j}}{d t}=\frac{1}{16 \pi} \int \epsilon_{j p q} x_{p}\left\langle\left(h_{a a}^{\mathrm{TT}} h_{a b, 0}^{\mathrm{TT}}\right)_{, b}-\frac{1}{2} h_{a b, a}^{\mathrm{TT}} h_{a b, 0}^{\mathrm{TT}}\right\rangle r^{2} d \Omega$.

An equivalent formula, obtained from Eq. (4.22) by integration by parts, is

$\frac{d S_{j}}{d t}=\frac{1}{16 \pi} \int\left\langle\epsilon_{j p q} h_{p a}^{\mathrm{TT}} h_{a q, 0}^{\mathrm{TT}}-\frac{1}{2} \epsilon_{j p q} x_{p} h_{a b, q}^{\mathrm{TT}} h_{a b, 0}^{\mathrm{TT}}\right\rangle r^{2} d \Omega$.

A word of interpretation is needed. This equation is correct only if the integral is evaluated in the asymptotic rest frame of the source. (Similarly for all previous formulae in this section.) As the source's linear momentum changes [Eq. (4.20)], its asymptotic rest frame gradually changes; and one must gradually change the reference frame in which one evaluates Eq. (4.22) (and all previous integrals).

Return to Eq. (4.22). An exceedingly long and tedious calculation, using techniques similar to those for evaluating the energy and momentum integrals, brings Eq. (4.22) into the forms

$$
\begin{aligned}
& \frac{d S_{j}}{d t}=\frac{i}{32 \pi} \sum_{l=2}^{\infty} \sum_{m}\left\langle{ } ^ { ( l ) } I ^ { l m * } \left\{\left[\frac{1}{2}(l+m)(l-m+1)\right]^{1 / 2(l+1)} I^{l m-1} \xi_{j}^{-1}\right.\right. \\
& \left.\left.+m^{(l+1)} I^{l m} \xi_{j}^{0}-\left[\frac{1}{2}(l-m)(l+m+1)\right]^{1 / 2(l+1)} I^{l m+1} \xi_{j}^{1}\right\}\right\rangle \\
& +\frac{i}{32 \pi} \sum_{l=2}^{\infty} \sum_{m}\left\langle^ { ( l ) } S ^ { l m * } \left\{\left[\frac{1}{2}(l+m)(l-m+1)\right]^{1 / 2(l+1)} S^{l m-1} \xi_{j}^{-1}\right.\right. \\
& \left.\left.+m^{(l+1)} S^{l m} \xi_{j}^{0}-\left[\frac{1}{2}(l-m)(l+m+1)\right]^{1 / 2(l+1)} S^{l m+1} \xi_{j}^{1}\right\}\right\rangle \text {; } \\
& \frac{d S_{j}}{d t}=\sum_{l=2}^{\infty} \frac{(l+1)(l+2)}{(l-1) l !(2 l+1) ! !}\left\langle\epsilon_{j p q}{ }^{(t)} g_{p A_{l-1}}{ }^{(l+1) g_{\alpha A_{l-1}}}\right\rangle \\
& +\sum_{l=2}^{\infty} \frac{4 l^{2}(l+2)}{(l-1)(l+1) !(2 l+1) ! !}\left\langle\epsilon_{j p q}{ }^{(l)} S_{p A_{l-1}}{ }^{(l+1)} S_{\alpha A_{l-1}}\right\rangle \text {. }
\end{aligned}
$$

As far as I know, general expressions such as these have not been given before; but the leading term (mass quadrupole) was given by Peters (1964), ${ }^{2}$ and the next two terms (current quadrupole and mass octupole) were given by Cooperstock and Booth (1969).

\section{E. Discussion}

Of what use are the above formulas? I view them as tools to be used in studying the generation of gravitational waves from explicit sources: Given a source, one identifies the local wave zone. Using any technique one can dream up (and this is the tough part of the analysis!), one calculates the time-changing multipole moments of the source. One then plugs into the above formulas to get the radiation field and the rate it carries off energy, momentum, and intrinsic angular momentum.

As an alternative application, one can calculate the radiation field of a given source (the tough task; above formulas not necessarily useful); one can use Eq. (4.10) or (4.11) to resolve it into multipole pieces; and one can then use the other formulas above to read off the energy, momentum, and angular momentum radiated.

Once the radiation field $h_{i j}^{\mathrm{TT}}$ is known in the local wave zone, one can propagate it on outwards to Earth using the propagation equation of the shortwave formalism [MTW, Sec. 35.14 and exercise 35.15; Thorne (1977) last section]. For typical situations the dominant parts of the waveforms (4.3) and (4.8) will remain highly accurate all the way to Earth, except for uninteresting phase shifts caused by background curvature and the waves' self-energy.

\section{MULTIPOLE MOMENTS EXPRESSED AS INTEGRALS OVER SOURCE}

\section{A. General sources}

For any asymptotically flat system one can introduce an asymptotically Minkowskii coordinate system in which the quantity

$$
\bar{h}^{\alpha \beta} \equiv-(-g)^{1 / 2} g^{\alpha \beta}+\eta^{\alpha \beta}
$$

\footnotetext{
${ }^{2}$ Note DeWitt's correction of an error in the Peters formula for the angular momentum density in the waves, as detailed on p. 992 of MTW.
} 
satisfies the de Donder gauge condition

$$
\bar{h}_{, \beta}^{\alpha \beta}=0 \text {; }
$$

see, e.g., Fock (1964, p. 193). In these de Donder coordinates the exact Einstein field equations take the form

$$
\square \bar{h}^{\alpha \beta}=-16 \pi \tau^{\alpha \beta},
$$

where $\square$ is the flat-space scalar wave operator $-\partial_{t}^{2}+\nabla^{2}$, and the "effective stress-energy" $\tau^{\alpha \beta}$ has the form

$\tau^{\alpha \beta}=(-g)\left(T^{\alpha \beta}+t_{\mathrm{LL}}^{\alpha \beta}\right)+(16 \pi)^{-1}\left[\bar{h}^{\alpha \mu}{ }_{, \nu} \bar{h}^{\beta \nu}{ }_{; \mu}-\bar{h}^{\alpha \beta}{ }_{, \mu \nu} \bar{h}^{\mu \nu}\right]$.

Here $t_{L L}^{\alpha \beta}$ is the Landau-Lifshitz pseudotensor [MTW Eq. (20.22), or Landau and Lifshitz (1971) Eq. (101.7)]. The effective stress-energy and its two individual pieces are coordinate-divergence-free

$$
\tau_{, \beta}^{\alpha \beta}=0 ;\left[(-g)\left(T^{\alpha \beta}+t_{\mathrm{LL}}^{\alpha \beta}\right)\right]_{, \beta}=0 .
$$

Far from the source, where $\left|\bar{h}^{\alpha \beta}\right| \ll 1, \bar{h}^{\alpha \beta}$ reduces to the trace-reversed metric perturbation: $\bar{h}^{\alpha \beta}=$ $=h^{\alpha \beta}-\frac{1}{2} \eta^{\alpha \beta} h$.

For systems with extremely strong internal gravitye.g., systems involving black holes - the de Donder coordinate system may not cover all of spacetime in a nonsingular manner. In the rest of Sec. V (but only here) we forego treating such systems; i.e., we restrict ourselves to systems which admit nonsingular spacetime-covering de Donder coordinates. We can then invert the Einstein field equations using the flatspace outgoing-wave Green's function $G_{j k \cdot p q}^{+}\left(x, x^{\prime}\right)$ [cf. Eqs. (5.2b) and (2.49)]

$$
\bar{h}_{j k}(x)=16 \pi \int G_{j k \cdot p q}^{+}\left(x, x^{\prime}\right) \tau_{p q}\left(x^{\prime}\right) d^{4} x^{\prime} .
$$

Note that this in fact is an integral equation for $\bar{h}_{j k}$, because $\tau_{p q}$ depends on $\bar{h}_{j k}$ [Eq. (5.3)].

We now restrict attention to field points $x$ in the local wave zone; we take the transverse-traceless part of the radiation field $\bar{h}_{j k}^{\mathrm{TT}}=h_{j k}^{\mathrm{TT}}$; and we use the factorized form $(2.50 \mathrm{c})$ of the Green's function to obtain

$$
\begin{aligned}
h_{j k}^{\mathrm{TT}}(x)= & 16 \pi i \sum_{\lambda l^{\prime} l m} \int d \omega\left(\operatorname{sgn}(\omega)\left[\Phi_{j k}^{+\omega \lambda l^{\prime} l m}(x)\right]^{\mathrm{TT}}\right. \\
& \left.\times \int\left[\Phi_{p q}^{S \omega \lambda l^{\prime} l m}\left(x^{\prime}\right)\right]^{*} \tau_{p q}\left(x^{\prime}\right) d^{4} x^{\prime}\right) .
\end{aligned}
$$

Again this is an integral equation; $\tau_{p q}$ depends on $h_{j k}^{\mathrm{TT}}$ as well as on other parts of the gravitational field. Of the basis outgoing-wave solutions $\Phi_{j k}^{+\omega \lambda l^{\prime \prime} l m}$ only those with $\lambda=2$ have nonzero TT part; and of these, the ones with $l^{\prime}=l-2, l, l+2$ contribute to the mass multipole, while the ones with $l^{\prime}=l-1, l+1$ contribute to the current multipole. More specifically, upon inserting into Eq. (5.6) expressions (2.45c) and (2.48c) for the $\Phi$ 's, and upon using Eqs. (2.33) and (2.34) to compute the TT parts of the pure-orbital harmonics $T_{j k}^{\lambda l^{\prime}, l m}$, and upon using the leading $1 / r$ term in expression $(2.47 \mathrm{~b})$ for $h^{+l}(\omega r)$, one obtains the standard radiation-zone expansion (4.3) for $h_{j k}^{\mathrm{TT}}$, and the following expressions for the multipole moments of the radiation field (where we have changed the names of the integration variables from $t^{\prime}$, $r^{\prime}, \Omega^{\prime}$ to $\left.t^{\prime}, r, \Omega\right)$ :

$$
\begin{aligned}
{ }^{(l)} I^{l m}(t)= & (-i)^{l+2} 8 \int e^{-i \omega\left(t-t^{\prime}\right)}\left[\left(\frac{(l+1)(l+2)}{2(2 l-1)(2 l+1)}\right)^{1 / 2}\left[T_{p q}^{2 l-2, l m}(\Omega)\right]^{*} j^{l-2}(\omega r)-\left(\frac{3(l-1)(l+2)}{(2 l-1)(2 l+3)}\right)^{1 / 2}\left[T_{p q}^{2 l, l m}(\Omega)\right]^{*} j^{l}(\omega r)\right. \\
& \left.+\left(\frac{l(l-1)}{2(2 l+1)(2 l+3)}\right)^{1 / 2}\left[T_{p q}^{2 l+2, l m}(\Omega)\right]^{*} j^{l+2}(\omega r)\right] \tau_{p q}\left(t^{\prime}, r, \Omega\right) r^{2} d \Omega d r d t^{\prime} d \omega \\
{ }^{(l)} S^{l m}(t)= & (-i)^{l+2} 8 \int e^{-i \omega\left(t^{\prime-t^{\prime}}\right)}\left[\left(\frac{l+2}{2 l+1}\right)^{1 / 2}\left[T_{p q}^{2 l-1, l m}(\Omega)\right]^{*} j^{l-1}(\omega r)\right. \\
& \left.-\left(\frac{l-1}{2 l+1}\right)^{1 / 2}\left[T_{p q}^{2 l+1, i m}(\Omega)\right]^{*} j^{l+1}(\omega r)\right] \tau_{p q}\left(t^{\prime}, r, \Omega\right) r^{2} d \Omega d r d t^{\prime} d \omega
\end{aligned}
$$

In these expressions the integral over frequency can be performed explicitly using the fact that the Fourier transform of a spherical Bessel function is a Legendre polynomial. The result is

$$
\begin{aligned}
{ }^{(l)} I^{l m}(t)= & 8 \pi(-1)^{l} \int r^{2} d \Omega d r\left\{\int _ { - 1 } ^ { + 1 } d \eta \left[\left(\frac{(l+1)(l+2)}{2(2 l-1)(2 l+1)}\right)^{1 / 2}\left[T_{p q}^{2 l-2, l m}(\Omega)\right]^{*} P^{l-2}(\eta)\right.\right. \\
& \left.\left.+\left(\frac{3(l-1)(l+2)}{(2 l-1)(2 l+3)}\right)^{1 / 2}\left[T_{p q}^{2 l}{ }^{l} l m(\Omega)\right]^{*} P^{l}(\eta)+\left(\frac{l(l-1)}{2(2 l+1)(2 l+3)}\right)^{1 / 2}\left[T_{p q}^{2 l+2, l \cdot m}(\Omega)\right]^{*} P^{l+2}(\eta)\right] \tau_{p q}(t-r \eta, r, \Omega)\right\}, \\
{ }^{(l)} S^{l m}(t)= & 8 \pi i(-1)^{l+1} \int r^{2} d \Omega d r\left\{\int _ { - 1 } ^ { + 1 } d \eta \left[\left(\frac{l+2}{2 l+1}\right)^{1 / 2}\left[T_{p q}^{2 l-1, l m}(\Omega)\right]^{*} P^{l-1}(\eta)\right.\right. \\
& \left.\left.+\left(\frac{l-1}{2 l+1}\right)^{1 / 2}\left[T_{p q}^{2 l+1, l m}(\Omega)\right]^{*} P^{l+1}(\eta)\right] \tau_{p q}(t-r \eta, r, \Omega)\right\}
\end{aligned}
$$

where $P^{l}$ is our unconventional notation for the Lengendre polynomial of order $l$. These types of gravitational source integrals involving Legendre polynomials were first constructed by Campbell, Macek, and Morgan (1977).

The corresponding source integrals for the STF moments $\mathcal{J}_{A_{l}}$ and $\delta_{A_{l}}$ can be constructed by inserting expressions (2.40) for $T^{2} l^{\prime}, l m$ into Eq. (5.7), and by then comparing with Eq. (4.7). (In making the comparison one should note that, because $\mathcal{Y}_{A_{l}}^{l m *}$ is STF on its $l$ indices, only the STF parts of the quantities contracted into $\mathcal{Y}_{A_{l}}^{l m *}$ can contribute to $I^{l m}$ and $S^{l m}$.) The result is 


$$
\begin{aligned}
& { }^{\left({ }^{\prime}\right)} g_{A_{l}}=\left[\frac { l ( l - 1 ) ( 2 l - 3 ) ! ! } { 2 \pi i ^ { l + 2 } } \int e ^ { - i \omega ( t - t ^ { \prime } ) } \left\{N_{A_{l-2}} \tau_{a_{l-1} a_{l}}\left(j^{l-2}-\frac{6(l-1)(2 l+1)}{(l+1)(2 l+3)} j^{l}+\frac{(l-1) l(2 l-1)}{(l+1)(l+2)(2 l+3)} j^{l+2}\right)\right.\right. \\
& +N_{A_{l-1}} \tau_{a_{l} j} n_{j}\left(\frac{6(2 l-1)(2 l+1)}{(l+1)(2 l+3)} j^{l}-\frac{2 l(2 l-1)(2 l+1)}{(l+1)(l+2)(2 l+3)} j^{l+2}\right)+N_{A_{l}} \tau_{j j}\left(-\frac{2(2 l-1)(2 l+1)}{(l+1)(2 l+3)} j^{l}-\frac{(2 l-1)(2 l+1)}{(l+1)(l+2)(2 l+3)} j^{l+2}\right) \\
& \left.\left.+N_{A_{l}} n_{j} n_{k} \tau_{j k}\left(\frac{(2 l-1)(2 l+1)}{(l+1)(l+2)} j^{l+2}\right)\right\} d \omega d^{3} x d t^{\prime}\right]^{\mathrm{STF}} \text {, } \\
& \text { (i) } S_{A_{l}}=\left[\frac{(l-1)(2 l-1) ! !}{2 \pi i^{i-1}} \int e^{-i \omega\left(t-t^{\prime}\right)}\right. \\
& \left.\times\left\{N_{A_{l-2}} \epsilon_{a_{l-1} p_{j}} n_{p} \tau_{j a_{l}}\left[j^{l-1}-\left(\frac{l-1}{l+2}\right) j^{l+1}\right]+\left(\frac{2 l+1}{l+2}\right) N_{A_{l-1}} \epsilon_{a_{l} p_{j}} n_{p} \tau_{j k} n_{k} j^{l+1}\right\} d \omega d^{3} x d t^{\prime}\right]^{\mathrm{STF}} .
\end{aligned}
$$

Here $j^{l^{\prime}} \equiv j^{l^{\prime}}(\omega r)$ and $\tau_{j k} \equiv \tau_{j k}\left(t^{\prime}, \mathrm{x}\right)$. These are the STF analogs of Eq. (5.7). The analogs of Eq. (5.8), involving Legendre functions rather than spherical Bessel functions, can be derived by performing the integral over $\omega$ in Eq. (5.9).

The source integrals (5.7)-(5.9) for $I^{l m}, S^{l m}, g_{A_{l}}$, and $\mathcal{S}_{A_{l}}$ are not particularly useful when the source has strong gravity and fast motions. This is because the integrals involve $\tau_{j k}$, which in turn depends on the gravitational field $\bar{h}_{\alpha \beta}$ [Eq. (5.3)]. It may be prohibitively difficult to compute $\bar{h}_{\alpha \beta}$ for insertion into the source integrals.

\section{B. Slow-motion sources}

We now specialize to slow-motion sources-i.e.', to sources which are confined to the deep interior of the near zone. For such sources

$\omega r \ll 1$ for $r$ such that $\tau_{\alpha \beta}$ is non-negligible in size, and

for $\omega$ such that non-negligible radiation

emerges at this frequency.

Hence, we can expand the spherical Bessel functions $j^{l^{\prime}}(\omega r)$ in powers of $\omega r$ [real part of Eqs. (2.47c)] and keep only the leading term

$$
j^{l^{\prime}}(\omega r)=\left[\left(2 l^{\prime}+1\right) ! !\right]^{-1}(\omega r)^{l^{\prime}}\left[1+O\left(\omega^{2} r^{2}\right)\right] .
$$

The dominant contribution to the mass moment ${ }^{(l)} I^{l m}$ comes from $l^{\prime}=l-2 ; l^{\prime}=l$ is down from it by $(\omega r)^{2}$, and $l^{\prime}=l+2$ is down by $(\omega r)^{4}$. The dominant contribution to the current moment ${ }^{(l)} S^{l m}$ comes from $l^{\prime}=l-1 ; l^{\prime}$ $=l+1$ is down by $(\omega r)^{2}$. Hence, aside from fractional errors in the integrands of order $(\omega r)^{2}$,

$$
\begin{aligned}
& { }^{(l)} I^{l m}(t)=\frac{8(-i)^{l+2}}{(2 l-3) ! !}\left(\frac{(l+1)(l+2)}{2(2 l-1)(2 l+1)}\right)^{1 / 2} \\
& \times \int(\omega r)^{l-2} e^{-i \omega\left(t-t^{\prime}\right)}\left[T_{p q}^{2 l-2, l m}(\Omega)\right]^{*} \\
& \times \tau_{p q}\left(t^{\prime}, \mathbf{x}\right) d^{3} x d t^{\prime} d \omega, \\
& { }^{(l)} S^{l m}(t)=\frac{8(-i)^{l+2}}{(2 l-1) ! !}\left(\frac{l+2}{2 l+1}\right)^{1 / 2} \\
& \times \int(\omega r)^{l-1} e^{-i \omega\left(t-t^{\prime}\right)}\left[T_{p Q}^{2 l-1, l m}(\Omega)\right]^{*} \\
& \times \tau_{p q}\left(t^{\prime}, \mathbf{x}\right) d^{3} x d t^{\prime} d \omega \text {. }
\end{aligned}
$$

We now perform the integrals over $\omega$ and $t^{\prime}$ using the relations

$$
\int(-i \omega)^{l^{\prime}} e^{-i \omega\left(t-t^{\prime}\right)} f\left(t^{\prime}\right) d t^{\prime} d \omega=2 \pi^{\left(l^{\prime}\right)} f(t) ;
$$

and we express $T_{p q}^{2 l^{\prime}, l m}(\Omega)$ in STF form using Eqs. (2.40). The result is

$$
\begin{aligned}
{ }^{(2)} I^{l m}(t)= & \frac{16 \pi}{(2 l+1) ! !}\left(\frac{(l-1) l(l+1)(l+2)}{2}\right)^{1 / 2} \\
& \times Y_{j k A_{l-2} l m *}^{l m *} \int X_{A_{l-2}} \tau_{j k}(t, \mathbf{x}) d^{3} x, \\
{ }^{(1)} S^{l m}(t)= & \frac{16 \pi}{(2 l+1) ! !}\left(\frac{2(l-1) l(l+2)}{l+1}\right)^{1 / 2} \\
& \times \epsilon_{p q j} y_{k q A}^{l m *} \int x_{p} X_{A_{l-2}} \tau_{j k}(t, \mathbf{x}) d^{3} x .
\end{aligned}
$$

By virtue of the "differential conservation laws" $\tau^{\alpha \beta}{ }_{, \beta}=0$ of the source-which we can rewrite

$$
\partial_{t} \tau_{00}=\tau_{0 j, j}, \quad \partial_{t} \tau_{0 j}=\tau_{j k, k}
$$

- the source satisfies the identities

$$
\begin{aligned}
&(l-1) l \tau_{j k} X_{A_{l-2}} \mathcal{Y}_{j k A}^{l m *}{ }_{l-2}^{l m} \\
&=\left(\partial_{t}{ }^{2} \tau_{00}\right) X_{A_{l}} Y_{A_{l}}^{l m *}-\left(\tau_{j k} X_{A_{l}}\right)_{, j k} \mathcal{Y}_{A_{l}}^{l m *} \\
&+2 l\left(\tau_{j k} X_{A_{l-1}}\right)_{, j} \mathcal{Y}_{k A_{l-1}^{l m *}}^{l m *} \\
&(l-1) \epsilon_{p q j} \mathcal{Y}_{k q A}^{l m *} \tau_{l-2} \tau_{j k} x_{p} X_{A_{l-2}} \\
&=-\epsilon_{p q j} \mathcal{Y}_{q A}^{l m *}\left(\partial_{t} \tau_{0 j}\right) x_{p} X_{A_{l-1}} \\
&+\epsilon_{p q j} \mathcal{Y}_{q A}^{l m *}\left(\tau_{j k} x_{p} X_{A_{l-1}}\right)_{, k} .
\end{aligned}
$$

By inserting these identities into Eqs. (5.14) and integrating out the divergences to zero, we obtain

$$
\begin{aligned}
I^{l m}= & \frac{16 \pi}{(2 l+1) ! !}\left(\frac{(l+1)(l+2)}{2(l-1) l}\right)^{1 / 2} y_{A_{l}}^{l m *} \int \tau_{00} X_{A_{l}} d^{3} x, \\
S^{l m}= & \frac{-32 \pi}{(2 l+1) ! !}\left(\frac{l(l+2)}{2(l-1)(l+1)}\right)^{1 / 2} \\
& \times y_{j A_{l-1}^{l m *}}^{l m} \int \epsilon_{j p q} x_{p}\left(-\tau_{0 q}\right) X_{A_{l-1}} d^{3} x .
\end{aligned}
$$

By then comparing with Eqs. (2.11), (2.24a), and (2.23b) we obtain

$$
I^{l m}=\frac{16 \pi}{(2 l+1) ! !}\left(\frac{(l+1)(l+2)}{2(l-1) l}\right)^{1 / 2} \int \tau_{00} Y^{l m *} r^{l} d^{3} x
$$




$$
\begin{aligned}
S^{l m}= & \frac{-32 \pi}{(2 l+1) ! !}\left(\frac{(l+2)(2 l+1)}{2(l-1)(l+1)}\right)^{1 / 2} \\
& \times \int \epsilon_{j p q} x_{p}\left(-\tau_{0 q}\right) Y_{j}^{l-1, l m * *} \gamma^{l-1} d^{3} x \\
= & \frac{32 \pi}{(2 l+1) ! !}\left(\frac{l+2}{2(l-1)}\right)^{1 / 2} \int\left(-\tau_{0 j}\right) Y_{j}^{B}, l m * \gamma^{l} d^{3} x .
\end{aligned}
$$

Similarly, by comparing Eqs. (5.17) with (4.7), and by noting that because $y_{A_{l}}^{l m *}$ is STF on its $l$ indices only the STF parts of the integrals in Eqs. (5.17a) and (5.17b) contribute to $I^{l m}$ and $S^{l m}$, we obtain

$$
\begin{aligned}
& g_{A_{l}}=\left[\int \tau_{00} X_{A_{l}} d^{3} x\right]^{\mathrm{STF}}, \\
& \S_{A_{l}}=\left[\int \epsilon_{a_{\imath}{ }^{p q}} x_{p}\left(-\tau_{0 Q}\right) X_{A_{l-1}} d^{3} x\right]^{\mathrm{ST} \mathrm{F}} .
\end{aligned}
$$

Thus, the mass moment $g_{A}(t)$ which characterizes the radiation field is equal to the $\mathrm{STF}$ part of the $\mathrm{lth}$ moment of the effective mass distribution $\tau_{00}$; and the current moment $\mathrm{S}_{A_{i}}(t)$ is equal to the STF part of the $(l-1)$ th moment of the effective angular momentum distribution $\epsilon_{j p q} x_{p}\left(-\tau_{0 q}\right)=\epsilon_{j p q} x_{p} \tau_{q}^{0}$. The general expressions (5.18) were derived in linearized theory by Mathews (1962). The mass quadrupole term dates back to Einstein (1918); and the current quadrupole and mass octupole terms, in rather different notation, are due to Papapetrou $(1962,1971)$.
Expressions (5.18) and (5.19) for the radiation moments are not exact. If $M$ is the mass of the source, $L$ is its characteristic size, $\chi=1 / \omega$ is the characteristic reduced wavelength or time scale of the radiation it emits, and $\pi_{d} \sim L\left|\tau_{00} / \tau_{0 q}\right|$ is the characteristic dynamical time scale for internal motions of the source, then Eqs. (5.18) and (5.19) make errors of magnitude

$$
\begin{aligned}
& \left|\delta I^{l m}\right| \sim\left|\delta g_{A_{l}}\right| \sim M L^{l}(L / \chi)^{2}, \\
& \left|\delta S^{l m}\right| \sim\left|\delta S_{A_{l}}\right| \sim M L\left(L / \chi_{d}\right)(L / \chi)^{2} ;
\end{aligned}
$$

cf. Eq. (5.11) and associated discussion. For typical systems

$$
\left|I^{l m}\right| \sim\left|g_{A_{l}}\right| \sim M L^{l}, \quad\left|S^{l m}\right| \sim\left|\mathfrak{S}_{A_{l}}\right| \sim M L^{l}\left(L / \chi_{d}\right) ;
$$

so that the fractional errors are of order $(L / \chi)^{2}$. However, for highly symmetrical systems the actual moments of interest may be much smaller than (5.21); and the fractional errors can be dangerously larger than $(L / \pi)^{2}$.

It is straightforward to derive from Eqs. (5.7) or (5.9) higher-order corrections to the slow-motion moments (5.18) and (5.19). One need only keep higherorder terms in the expansions of $j^{l^{\prime}}(\omega r)$. By keeping all terms in the expansions one obtains the following exact infinite series for the moments of a slow-motion source

$$
\begin{aligned}
& I^{l m}=\frac{16 \pi}{(2 l+1) ! !}\left(\frac{(l+1)(l+2)}{2(l-1) l}\right)^{1 / 2} \int \tau_{00} Y^{l m *} r^{l} d^{3} x \\
& +\sum_{k=0}^{\infty} \frac{16 \pi}{2^{k} k !(2 l+2 k+1) ! !}\left(\partial_{t}\right)^{2 k} \int \tau_{p q} \gamma^{l+2 k}\left[\frac{(2 l+2 k+1)}{2(k+1)}\left(\frac{(l+1)(l+2)}{2(2 l-1)(2 l+1)}\right)^{1 / 2} T_{p q}^{2 l-2, l m *}\right. \\
& \left.+\left(\frac{3(l-1)(l+2)}{(2 l-1)(2 l+3)}\right)^{1 / 2} T_{p q}^{2} l, l m *+\frac{2 k}{2 l+2 k+3}\left(\frac{l(l-1)}{2(2 l+1)(2 l+3)}\right)^{1 / 2} T_{p q}^{2} l+2, l m *\right] d^{3} x, \\
& S^{l m}=\frac{-32 \pi}{(2 l+1) ! !}\left(\frac{(l+2)(2 l+1)}{2(l-1)(l+1)}\right)^{1 / 2} \int \epsilon_{j p q} x_{p}\left(-\tau_{0 q}\right) Y_{j}^{l-1, l m *_{\gamma^{l-1}} d^{3} x} \\
& +\sum_{k=0}^{\infty} \frac{16 \pi i}{2^{k} k !(2 l+2 k+1) ! !}\left(\partial_{t}\right)^{2 k+1} \int \tau_{p q} \gamma^{l+2 k+1}\left[\frac{1}{2(k+1)}\left(\frac{l+2}{2 l+1}\right)^{1 / 2} T_{p q}^{2 l-1, l m *}+\frac{1}{2 l+2 k+3}\left(\frac{l-1}{2 l+1}\right)^{1 / 2} T_{p q}^{2 l+1, l m *}\right] d^{3} x ; \\
& \boldsymbol{g}_{A_{l}}=\left[\int\left(\tau_{00} X_{A_{l}}+\sum_{k=0}^{\infty}\left(r \partial_{t}\right)^{2 k}\left(A^{l k} r^{2} X_{A_{l-2}} \tau_{a_{l-1} a_{l}}+B^{l k} X_{A_{l-1}} \tau_{a_{l} l^{j}} x_{j}+C^{l k} X_{A_{l}} \tau_{j j}+D^{l k} X_{A_{l}} n_{p} n_{q} \tau_{p q}\right)\right) d^{3} x\right]^{\mathrm{STF}} \text {; }
\end{aligned}
$$

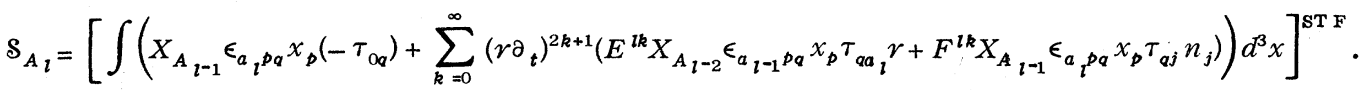

In expressions (5.23) the coefficients $A^{t k}, \ldots, F^{l k}$ are

$$
\begin{aligned}
& A^{l k}=\frac{(l-1) l(2 l+1) ! !}{2^{k} k !(2 l+2 k+3) ! !}\left[\frac{(2 l+2 k+1)(2 l+2 k+3)}{2(2 l-1)(2 l+1)(k+1)}+\frac{6(l-1)(2 l+2 k+3)}{(l+1)(2 l-1)(2 l+3)}+\frac{2(l-1) l k}{(l+1)(l+2)(2 l+1)(2 l+3)}\right], \\
& B^{l k}=-\frac{(l-1) l(2 l+1) ! !}{(l+1)(2 l+3) 2^{k-1} k !(2 l+2 k+1) ! !}\left[3+\frac{2 l k}{(l+2)(2 l+2 k+3)}\right] \\
& C^{l k}=\frac{(l-1) l(2 l+1) ! !}{(l+1)(2 l+3) 2^{k-1} k !(2 l+2 k+1) ! !}\left[1-\frac{k}{(l+2)(2 l+2 k+3)}\right] \\
& D^{l k}=\frac{(l-1) l(2 l+1) ! ! k}{(l+1)(l+2) 2^{k-1} k !(2 l+2 k+3) ! !} \\
& E^{l k}=\frac{(l-1)(2 l-1) ! !}{2^{k} k !(2 l+2 k+3) ! !}\left[\left(\frac{l-1}{l+2}\right)+\frac{(2 l+2 k+3)}{2(k+1)}\right]
\end{aligned}
$$




$$
F^{l k}=-\frac{(l-1)(2 l+1) ! !}{(l+2) 2^{k} k !(2 l+2 k+3) ! !} .
$$

Although the exact source integrals (5.22) and (5.23) were derived using Fourier-transform techniques [Eqs. (5.6) $-(5.9)]$, they are valid independently of the Fourier transformability of $h_{j k}^{\mathrm{TT}}$. Fourier transforms are nothing but a trick to simplify algebraic manipulations that can be performed equally well in the time domain.

It is straightforward to verify that, if the multipole moment $\mathfrak{T}_{A_{l}}\left(\equiv g_{A_{l}}\right.$ or $\left.S_{A_{l}}\right)$ is the largest contributor to the radiation field (4.8), then one can ignore "gravitational pseudotensor" contributions to $\mathfrak{T}_{A_{l}}$ from radii $\pi \sim r$; i.e., one can confine the integrals (5.18), (5.19), (5.22), (5.23) for $\mathfrak{M}_{A_{l}}$ to radii deep inside the near zone. In doing so, one makes errors $\left|\delta \Re_{A_{l}}\right| \lesssim(M / \chi)\left|\mathfrak{T}_{A_{l}}\right|$; cf. Eq. (9.34) below.

As we shall see below, Eqs. (5.22) and (5.23) can be used to generate post-Newtonian and post-post-Newtonian formulas for the radiative moments.

\section{Newtonian sources}

Specialize now to slow-motion sources with weak internal gravity and small internal stresses. Characterize the slow motion by a velocity parameter

$v \sim \begin{aligned} & \text { maximum of typical } \\ & \text { internal values of }\end{aligned}\left\{\frac{\left|T_{0 j}\right|}{\left|T_{00}\right|}, \frac{\left|T_{\alpha \beta, 0}\right|}{\left|T_{\alpha \beta, j}\right|}\right\} ;$

characterize the weak gravity by

$$
\epsilon \equiv \text { maximum over entire source of } \bar{h}_{00} \text {; }
$$

and characterize the small stresses by

$$
S \equiv \text { maximum over entire source of }\left|T_{j k}\right| /\left|T_{00}\right| \text {. }
$$

One obtains the Newtonian theory of gravity by (i) beginning in de Donder gauge [Eqs. (5.1) - (5.4)]; (ii) restricting attention to sources with

$$
\epsilon \sim(L / \chi)^{2} \sim v^{2} \sim S \ll 1 ;
$$

(iii) restricting attention to the near-zone neighborhood of the source, $r \leqslant L$; and (iv) expanding the equations of motion (5.4) to lowest order in $\epsilon$ and linearizing the Einstein field equations, (5.2b); see, e.g., Chap. 18 of MTW.

For Newtonian sources, the theory of gravitationalwave generation is obtained by carrying out the procedures of Secs. V.A and V.B, and ignoring fractional corrections of order $\epsilon \sim(L / X)^{2} \sim v^{2} \sim S$. The result is Eqs. (5.18) and (5.19) for the multipole moments of the radiation field, with $\tau_{00}=\rho=$ (Newtonian mass density) and $-\tau_{0 j}=\rho v_{j}=$ (Newtonian momentum density):

$$
\begin{aligned}
I^{l m}= & \frac{16 \pi}{(2 l+1) ! !}\left(\frac{(l+1)(l+2)}{2(l-1) l}\right)^{1 / 2} \int \rho Y^{l m *} \gamma^{2} d^{3} x, \\
S^{l m}= & \frac{-32 \pi}{(2 l+1) ! !}\left(\frac{(l+2)(2 l+1)}{2(l-1)(l+1)}\right)^{1 / 2} \\
& \times \int\left(\epsilon_{j p q} x_{p} \rho v_{q}\right) Y_{j}^{l-1, l m *} r^{l-1} d^{3} x
\end{aligned}
$$

$$
\begin{aligned}
& g_{A_{l}}=\left[\int \rho X_{A_{l}} d^{3} x\right]^{\mathrm{STF}}, \\
& \mathcal{S}_{A_{l}}=\left[\int\left(\epsilon_{a_{l} p Q} x_{p} \rho v_{q}\right) X_{A_{l-1}} d^{3} x\right]^{\mathrm{STF}} .
\end{aligned}
$$

Of course, the general expressions (5.27) are familiar from Mathews (1962) and the leading terms are familiar from Einstein (1918) and Papapetrou $(1962,1971)$. Note that the integrands of these source integrals are free of any reference to the gravitational field. They depend only on the source's material mass density and angular momentum density.

For typical Newtonian sources the multipole moments will have magnitudes (5.21) and will therefore produce radiation fields of strength

$$
\begin{aligned}
& \left(h_{j k}^{\mathrm{TT}}\right)_{\text {mass } l \text {-pole }} \sim(M / r)(L / \chi)^{l}, \\
& \left(h_{j k}^{\mathrm{TT}}\right)_{\text {current } l \text {-pole }} \sim(M / r)(L / \chi)^{l+1},
\end{aligned}
$$

where we have assumed that the source's dynamical time scale and gravitational-wave time scale are the same, $x_{d} \sim \chi$. For such sources, the Newtonian fractional errors $\epsilon \sim(L / X)^{2} \sim v^{2} \sim S$ in computing the various moments are such that

$$
\begin{aligned}
& \left|\frac{\left(h_{j k}^{\mathrm{T}}\right)_{\text {mass } l-\text { pole }}}{\text { error in }\left(h_{j k}^{\mathrm{TT}}\right)_{\text {mass quadrupole }}}\right| \sim\left(\frac{x}{L}\right)^{4-l}, \\
& \left|\frac{\left(h_{j k}^{\mathrm{TT}}\right)_{\text {current } l \text {-pole }}}{\text { error in }\left(h_{j k}^{\mathrm{TT}}\right)_{\text {mass quadrupole }}}\right| \sim\left(\frac{x}{L}\right)^{3-l} .
\end{aligned}
$$

Thus, only the mass quadrupole, mass octupole, and current quadrupole fields are larger than the dominant (mass quadrupole) error.

\section{Post-Newtonian sources}

For sources with $\epsilon \sim(L / \chi)^{2} \sim\left(L / x_{d}\right)^{2} \sim v^{2} \sim S$ small, but not extremely small, one may want to compute the radiation field with higher accuracy. One can do so using a post-Newtonian wave-generation formalism derived by Epstein and Wagoner (1975) and Wagoner (1977). [See Turner and Wagoner (1979) for an application of the formalism to collapsing stars.]

The Epstein-Wagoner procedure for deriving this formalism is the same as in the Newtonian case: (i) One constructs a near-zone post-Newtonian formalism for analyzing the source's motions; and one forces the formalism to satisfy the de Donder gauge condition (5.2a) to post-Newtonian order. (ii) Then one computes the radiative multipole moments, accurate to postNewtonian order [fractional errors $\sim(L / x)^{4}$ ] from Eqs. (5.22) and (5.23).

The resulting expressions for the multipole moments are

$$
\begin{aligned}
I^{l m}= & \frac{16 \pi}{(2 l+1) ! !}\left(\frac{(l+1)(l+2)}{2(l-1) l}\right)^{1 / 2} \\
& \times \int\left\{\tau_{00} Y^{l m *}+\frac{1}{2}\left(\frac{(l-1) l(2 l+1)}{2 l-1}\right)^{1 / 2} T_{j k}^{2 l-2, l m *} \tau_{j k}\right. \\
& \left.+\left(\frac{6 l(l-1)^{2}}{(l+1)(2 l-1)(2 l+3)}\right)^{1 / 2} T_{j k}^{2 l, l m *} \tau_{j k}\right\} V^{l} d^{3} x,
\end{aligned}
$$




$$
\begin{aligned}
S^{l m}= & \frac{-32 \pi}{(2 l+1) ! !}\left(\frac{(l+2)(2 l+1)}{2(l-1)(l+1)}\right)^{1 / 2} \\
\times & \left\{\epsilon_{j p q} n_{p}\left(-\tau_{0 Q}\right) Y_{j}^{l-1, l m *}-\frac{i[2(l-1)(l+1)]^{1 / 2}}{2(2 l+1)}\right. \\
& \left.\times\left[\frac{1}{2} T_{j k}^{2 l-1, l m *}+\frac{1}{2 l+3}\left(\frac{l-1}{l+2}\right)^{1 / 2} T_{j k}^{l l+1, l m} *\right] r \partial_{t} \tau_{j k}\right\} r^{l} d^{3} x ;
\end{aligned}
$$

$$
\begin{aligned}
\mathscr{g}_{A_{l}}=\left[\int(\right. & \tau_{00} X_{A_{t}}+A^{l 0} \gamma^{2} X_{A_{l-2}} \tau_{a_{l-1} a_{l}} \\
& \left.\left.+B^{l 0} X_{A_{l-1} \tau_{a_{l} j}} x_{j}+C^{l 0} X_{A_{l}} \tau_{j j}\right) d^{3} x\right]^{\mathrm{STF}},
\end{aligned}
$$

$$
\begin{aligned}
S_{A_{l}}=\left[\int(\right. & X_{A_{l-1}} \epsilon_{a_{l} j k} x_{j}\left(-\tau_{0 k}\right)+E^{l 0} X_{A_{l-2}} \epsilon_{a_{l-1} j k} x_{j} r^{2} \partial_{t} \tau_{k a_{l}} \\
& \left.\left.+F^{l 0} X_{A_{l-1}} \epsilon_{a_{l} j k} x_{j} r \partial_{t} \tau_{k p} n_{p}\right) d^{3} x\right]^{\mathrm{sTF}}
\end{aligned}
$$

Here

$$
\begin{aligned}
& A^{l 0}=\frac{(l-1) l(l+9)}{2(l+1)(2 l+3)}, \quad B^{l 0}=-\frac{6(l-1) l}{(l+1)(2 l+3)}, \\
& C^{l 0}=\frac{2(l-1) l}{(l+1)(2 l+3)}, \quad E^{l 0}=\frac{(l-1)(l+4)}{2(l+2)(2 l+3)}, \\
& F^{l 0}=-\frac{(l-1)}{(l+2)(2 l+3)}
\end{aligned}
$$

[cf. Eq. (5.24)]. In these expressions $\tau_{\alpha \beta}$ is the effective stress-energy tensor (5.3), evaluated at post-Newtonian order in the post-Newtonian de Donder gauge [Eqs. (37) of Epstein and Wagoner (1975), with indices lowered using the Minkowskii metric]. These $\tau_{\alpha \beta}$ depend on the source's Newtonian gravitational potential $\Phi$, but they are free of any reference to the gravitational-wave field $h_{j k}^{\mathrm{TT}}$.

For typical post-Newtonian sources the multipole moments will have magnitudes (5.21) and will therefore produce radiation fields of strength (5.29). When this is the case, the post-Newtonian fractional errors $(L / x)^{4}$ in computing the various moments are such that

$$
\begin{aligned}
& \left|\frac{\left(h_{j k}^{\mathrm{TT}}\right)_{\text {mass }} l \text {-pole }}{\text { (error in } \left.h_{j k}^{\mathrm{TT} T}\right)_{\text {mass quadrupole }}}\right| \sim\left(\frac{x}{L}\right)^{6-l}, \\
& \left|\frac{\left(h_{j k}^{\mathrm{TT}}\right)_{\text {currept } l \text {-pole }}}{\text { error in }\left(h_{j k}^{\mathrm{TT}}\right)_{\text {mass quadrupoie }}}\right| \sim\left(\frac{x}{L}\right)^{5-l} .
\end{aligned}
$$

If one is willing to drop all terms in $h_{j k}^{\mathrm{TT}}$ smaller than or comparable to the (mass quadrupole) errors, then for such sources one need only compute mass multipoles of order $l \leqslant 5$ and current multipoles of $l \leqslant 4$; and in doing the computations one need keep the post-Newtonian corrections only in the mass 2-pole and 3-pole and in the current 2-pole. [For the other moments the Newtonian expressions (5.27) and (5.28) produce radiation fields accurate to post-Newtonian order.] This is the procedure followed by Epstein and Wagoner (1975); see also Wagoner (1977).

On the other hand, for some special sources [e.g., torsional oscillations of neutron stars, Schumaker and Thorne (1980); also the collapse of a very slowly rotating star, Turner and Wagoner (1979)], the mass quadrupole moment will be strongly suppressed; Eqs.
(5.34) will not apply; and one will be justified in keeping post-Newtonian corrections in one or mure of the higher moments.

\section{E. Post-post-Newtonian sources and beyond}

If one wished, one could construct a post-post-Newtonian wave-generation formalism by the same procedure used at Newtonian and post-Newtonian orders. The first step would be to reformulate the Chandrasekhar-Nutku (1969) near-zone post-post-Newtonian formalism in de Donder gauge, and evaluate the effective stress energy $\tau^{\alpha \beta}$ [Eq. (5.3)] in terms of the variables of that formalism. Then the radiative multipole moments, to post-post-Newtonian accuracy [fractional errors $\left.\sim(L / x)^{6}\right]$ will be given by Eqs. (5.22) and (5.23) with the $k=0$ and $k=1$ terms kept, and $k \geqslant 2$ discarded.

In principle one could proceed beyond post-post-Newtonian order by the same procedure; but in doing so one would have to face, and carefully handle, issues of radiation reaction in the source; see Chandrasekhar and Esposito (1970).

\section{CONCLUSION}

In Part One of this paper we have examined the multipole structure of the generic gravitational-wave field of an arbitrary source. In doing so, we placed only one very mild restriction on the nature of the source: that it be "isolated"; i.e., that it possess a local wave zone.

However, when we derived formulas for the coupling of the multipole waves to their source, we had to impose a much stronger restriction: that the source be coverable by a single nonsingular de Donder coordinate system. Moreover, the resulting formulas are not terribly easy to apply (except in the Newtonian and postNewtonian limits), because the calculations must be performed in a de Donder coordinate system-which one may not have readily available in a given theoretical study - and because the integrands of the source integrals involve the gravitational-wave field itself $\left(\tau_{\alpha \beta}\right.$ depends on $\left.h_{j k}^{\mathrm{TT}}\right)$. Obviously, a better theory of coupling to the source would be useful.

In Part Two a new coupling formalism will be presented. It is valid for sources with arbitrarily strong internal gravity (including black holes); but it requires slow motions, $L / x \ll 1$.

\section{PART TWO. SLOW-MOTION SOURCES OF GRAVITATIONAL WAVES}

\section{INTRODUCTION}

In 1969, James Ipser (1970) computed the gravitational radiation emitted by a slightly deformed, slowly rotating, fully relativistic neutron star. His result was somewhat startling: He found that the multipole moments which governed the radiation field were precisely the same as the "mass multipoles" which one reads off the star's weak-field near-zone Newtonian potential. This led Ipser to conjecture that one can compute the gravitational waves from any strong-field slow-motion system by first computing (using full nonlinear general relativity) the system's asymptotic 
Newtonian potential, by reading the source's multipole moments off that potential, and by then inserting those moments into the gravitational-wave formulas of Part IV.

Soon thereafter, while writing the first draft of Chap. 36 of MTW, I found what I thought was a simple proof of Ipser's conjecture. That proof appears in the preliminary versions of MTW [Misner et al. $(1970,1971)]$ and is referred to in my review article with Bill Press on gravitational-wave astronomy [Press and Thorne (1972)). However, much to my horror, in March 1973 while checking page proofs of the final version of MTW, I found a subtle but fatal flaw in my proof of Ipser's conjecture. After much agony I managed to rewrite the relevant material [Secs. 36.7 and 36.10 of Misner et al . (1973)] with a restriction to sources that have weak internal gravity-and without changing by even one the total number of lines of text.

In Part $\mathrm{T}$ wo of this article I shall try to redeem myself by presenting a correct formulation and proof of Ipser's conjecture. This formulation will avoid the concept of the asymptotic Newtonian potential of a source; in its place will appear a prescription for reading the multipole moments of a source off its nearzone general relativistic metric. However, in all other respects the formalism will conform to Ipser's original ideas.

Part Two of this paper consists of five sections. The first four (Secs. VIII-XI) develop foundations for the strong-field, slow-motion wave-gene ration formalism. The last (Sec. XII) presents the formalism itself and describes a few applications.

Each of the four foundations is a derivation of the vacuum exterior gravitational field of a general isolated system. Section VIII derives that field for timedependent systems in linearized theory. Section IX derives it in the near zone of slow-motion timedependent systems in full general relativity using de Donder coordinates, and also matches that near-zone solution onto outgoing waves in the radiation zone. Section X specializes to time-independent gene ral relativistic systems in de Donder coordinates; and Sec. XI extends the time-independent general relativistic case to any "asymptotically Cartesian and masscentered" (ACMC) coordinate system.

For a more detailed overview see Sec. I.B, Box 2, and the table of contents - all in Part One of this article.

\section{LINEARIZED THEORY}

Here we express, in terms of time-dependent multipole moments, the linearized external gravitational field of an arbitrary isolated system. Similar expressions, but in different notation, have been given by Sachs and Bergmann (1958), Sachs (1961), Pirani (1964), and Campbell and Morgan (1971). The notation used here is designed to fit in with that of Part One and with the remainder of this paper.

In linearized theory (e.g., Chap. 18 of MTW), the metric of spacetime $g_{\alpha \beta}$ is written as the Minkowskii metric $\eta_{\alpha \beta}$ plus a small perturbation $g_{\alpha \beta}^{1}$ :

$$
g_{\alpha \beta}=\eta_{\alpha \beta}+g_{\alpha \beta}^{1} \text {. }
$$

We shall denote by $\gamma_{\alpha \beta}^{1}$ the trace-reversed perturbation

$$
\gamma_{\alpha \beta}^{1} \equiv g_{\alpha \beta}^{1}-\frac{1}{2} \eta_{\alpha \beta} \eta^{\mu \nu} g_{\mu \nu}^{1} .
$$

(The reason for our "superscript 1" notation will become clear in Sec. IX. The nature of our coordinate system and basis vectors, and the rules for raising and lowering indices, are discussed in Sec. I.C.)

We introduce Lorentz gauge $\gamma^{1 \alpha \beta}{ }_{, \beta}=0$ for our gravitational field. Then, expressed in terms of covariant components, the gauge conditions and linearized vacuum field equations (Eqs. 18.8 of MTW) read

$$
\begin{aligned}
& \gamma_{\alpha 0,0}^{1}=\gamma_{\alpha j, j}^{1}, \\
& \square \gamma_{\alpha \beta}^{1} \equiv-\gamma_{\alpha \beta, 00}^{1}+\gamma_{\alpha \beta, j j}^{1}=0 .
\end{aligned}
$$

We seek the most general symmetric gravitational field $\gamma_{\alpha \beta}^{1}=\gamma_{\beta \alpha}^{1}$ which satisfies these equations, and which has only outgoing waves (no incoming waves) at infinity; and we write that field as a sum over its multipole components.

The general outgoing-wave solution to the field equation $\square \gamma_{\alpha \beta}^{1}=0$ in multipole notation has the following form [see Eq. (2.51), whe re we must set $\epsilon=+1$ (outgoing waves) and we must make the identifications $\left.\gamma_{00}^{1}=F, \gamma_{0 j}^{1}=V_{j}, \gamma_{j k}^{1}=U_{j k}\right]$ :

$$
\begin{aligned}
& \gamma_{\mathrm{OO}}^{1}=\sum_{l=0}^{\infty}\left[r^{-1} \alpha_{A_{l}}(t-r)\right]_{A_{l}}, \\
& \gamma_{0 j}^{1}=\sum_{l=1}^{\infty}\left\{\left[r^{-1} \otimes_{j A_{l-1}}(t-r)\right]_{A_{l-1}}\right. \\
& \left.+\left[r^{-1} \epsilon_{j p q} \mathfrak{e}_{q A_{l-1}}(t-r)\right]_{, p A_{l-1}}\right\} \\
& +\sum_{l=0}^{\infty}\left[r^{-1} D_{A_{l}}(t-r)\right]_{, j A_{l}}, \\
& \gamma_{j k}^{1}=\sum_{l=0}^{\infty} \delta_{j k}\left[r^{-1} \mathcal{E}_{A_{l}}(t-r)\right]_{A_{l}} \\
& +\sum_{l=2}^{\infty}\left\{\left[r^{-1} \Im_{j k A_{l-2}}(t-r)\right]_{, A_{l-2}}\right. \\
& \left.+\left[r^{-1} \epsilon_{p q(j} S_{k) \alpha A_{l-2}}(t-r)\right]_{, p A_{l-2}}\right\} \\
& +\sum_{l=1}^{\infty}\left[\left[r^{-1} \mathcal{K}_{j A_{l-1}}(t-r)\right]_{, k A_{l-1}}\right. \\
& \left.+\left[r^{-1} \epsilon_{j p q} \partial_{q A_{l-1}}(t-r)\right]_{, k p A_{l-1}}\right]^{S} \\
& +\sum_{l=0}^{\infty}\left[r^{-1} \mathcal{K}_{A_{l}}(t-r)\right]_{, j k A_{l}} \text {. }
\end{aligned}
$$

Here $\epsilon_{i j k}$ is the completely antisymmetric Levi-Civita tensor; the capital script quantities are the multipole moments, which are arbitrary functions of retarded time $t-r$ and are symmetric and trace-free (STF) on all their tensor indices; and all other details of notation are explained in Sec. I.C. The gauge conditions (8.3a) place the following constraints on the STF tensors appearing in the solution (8.4):

$$
\begin{aligned}
& \mathbb{B}_{A_{l}}=\dot{Q} A_{A_{l}}-\ddot{D}_{A_{l}}, \quad \mathcal{F}_{A_{l}}=\ddot{Q}_{A_{l}}-\dddot{D}_{A_{l}}-\frac{1}{2} \ddot{\mathfrak{K}}_{A_{l}}, \\
& \mathcal{E}_{A_{l}}=\dot{D}_{A_{l}}-\frac{1}{2} \mathfrak{H}_{A_{l}}-\ddot{\mathfrak{K}}_{A_{l}}, \quad \mathrm{~S}_{A_{l}}=2 \dot{\mathrm{e}}_{A_{l}}-\ddot{\jmath}_{A_{l}} .
\end{aligned}
$$


Here a dot means time derivative. Because for $l=0$ all STF coefficients in Eqs. (8.4) are absent except $a, \mathfrak{D}, \mathcal{E}$, and $\mathcal{K}$, and for $l=1, \mathcal{F}$ and $\mathcal{S}$ are absent, Eqs. (8.5) for $l=0$ and 1 imply

$$
\begin{aligned}
& \dot{a}-\ddot{D}=0, \quad \mathcal{E}-\dot{D}+\ddot{\mathfrak{K}}=0, \\
& \dot{\mathfrak{e}}_{j}-\frac{1}{2} \ddot{\partial}_{j}=0, \quad \ddot{a}_{j}-\dddot{\mathscr{D}}_{j}-\frac{1}{2} \ddot{\mathfrak{C}}_{j}=0 .
\end{aligned}
$$

By evaluating the surface integrals (20.6) of MTW [and in the evaluation using $\bar{h}_{\alpha \beta}=\gamma_{\alpha \beta}^{1}$, Eqs. (8.5), (8.6), (2.3), and the symmetric, trace-free nature of the capital script tensors] we learn that the source has total four-momentum

$$
P^{0}=\frac{1}{4}(Q-\dot{D}), \quad P^{i}=-\frac{1}{4}\left(\dot{Q}_{i}-\ddot{D}_{i}-\frac{1}{2} \dot{\mathcal{K}}_{i}\right) .
$$

Thus, the first and last of Eqs. (8.6) are the law of four-momentum conservation. Henceforth we shall restrict attention to sources with timelike four-momentum; and by a Lorentz boost (Sec. A.1 of Box 18.2 of MTW) we shall put ourselves in the rest frame of the source, where

$$
P_{i}=-\frac{1}{4}\left(\dot{Q}_{i}-\ddot{D}_{i}-\frac{1}{2} \dot{\mathcal{C}}_{i}\right)=0 .
$$

In this reference frame, from Eqs. (20.8) and (20.11) of MTW we learn that the source's center of mass is offset from the origin of coordinates by the vector displacement

$$
Y_{i}=-(\boldsymbol{Q}-\dot{D})^{-1}\left(\boldsymbol{Q}_{i}-\dot{\mathfrak{D}}_{i}-\frac{1}{2} \mathfrak{H C}_{i}\right) .
$$

We now translate our origin of coordinates to the center of mass, thereby achieving

$$
a_{i}-\dot{D}_{i}-\frac{1}{2} \Re_{i}=0 \text {. }
$$

Next we simplify our expression for the gravitational field by the change of gauge (infinitesimal coordinate transformation; part A2 of Box 18.2 of MTW)

$$
\left(\gamma_{\alpha \beta}^{1}\right)_{n e w}=\left(\gamma_{\alpha \beta}^{1}\right)_{o 1 d}+\xi_{\alpha, \beta}+\xi_{\beta, \alpha}-\eta_{\alpha \beta} \xi_{, \mu}^{\mu},
$$

where the generating functions $\xi_{\alpha}$ are given by

$$
\begin{aligned}
\xi_{0}=\sum_{l=0}^{\infty}\left[-r^{-1} \mathcal{D}_{A_{l}}(t-r)\right]_{A_{l}}+\left[\frac{1}{2} r^{-1} \dot{\mathcal{K}}_{A_{l}}(t-r)\right]_{A_{l}}, \\
\xi_{j}=-\sum_{l=1}^{\infty}\left\{\left[\frac{1}{2} r^{-1} \mathcal{K}_{j A_{l-1}}(t-r)\right]_{A_{l-1}}\right. \\
\left.+\left[\frac{1}{2} r^{-1} \epsilon_{j p q} \mathcal{J}_{q A_{l-1}}\right]_{p A_{l-1}}\right\} \\
-\sum_{l=0}^{\infty}\left[\frac{1}{2} r^{-1} \mathcal{K}_{A_{l}}(t-r)\right]_{j A_{l}} .
\end{aligned}
$$

(Note that because $\square \xi_{\alpha}=0$, this gauge change preserves the de Donder gauge condition.) This gauge change brings to zero the following functions in the gravitational field of Eq. (8.4):

$$
D_{A_{l}}=\mathfrak{K}_{A_{l}}=\mathfrak{d}_{A_{l}}=\mathfrak{K}_{A_{l}}=0 \text {. }
$$

We now change notation, setting

$$
\begin{aligned}
& a_{A_{l}}=(-1)^{l}(4 / l !) \mathfrak{g}_{A_{l}}, \\
& \mathfrak{e}_{A_{l}}=(-1)^{l}[4 l /(l+1) !] \mathfrak{S}_{A_{l}},
\end{aligned}
$$

and thereby obtain our final form for $\gamma_{\alpha \beta}^{1}$ [Eqs. (8.4),

$$
\begin{aligned}
&(8.5),(8.6),(8.8 \mathrm{~b}),(8.10),(8.11)]: \\
& \gamma_{00}^{1}=\frac{4 \mathrm{~g}}{r}+\sum_{l=2}^{\infty}(-1)^{l} \frac{4}{l !}\left[r^{-1} g_{A_{l}}(t-r)\right]_{A_{l}}, \\
& \gamma_{0 j}^{1}= \frac{-2 \epsilon_{j p q} \S_{p} n_{q}}{r^{2}} \\
&-\sum_{l=2}^{\infty}(-1)^{l} \frac{4 l}{(l+1) !}\left[r^{-1} \epsilon_{j p q} \delta_{p A_{l-1}}(t-r)\right]_{, q A_{l-1}} \\
&+\sum_{l=2}^{\infty}(-1)^{l} \frac{4}{l !}\left[r^{-1} \dot{g}_{j A_{l-1}}(t-r)\right]_{, A_{l-1}}, \\
& \gamma_{j k}^{1}= \sum_{l=2}^{\infty}(-1)^{l} \frac{4}{l !}\left[r^{-1} \ddot{g}_{j k A_{l-2}}(t-r)\right]_{A_{l-2}} \\
&-\sum_{l=2}^{\infty}(-1)^{l} \frac{8 l}{(l+1) !}\left[r^{-1} \epsilon_{p q(j} \dot{s}_{k) p A_{l-2}}(t-r)\right]_{, q A_{l-2}}
\end{aligned}
$$

Here $n_{j}=x_{j} / r$ [cf. Eq. (1.5)]. The corresponding linear correction to the metric is [cf. Eq. (8.2)]

$$
\begin{aligned}
g_{00}^{1}= & \frac{2 \boldsymbol{g}}{r}+\sum_{l=2}^{\infty}(-1)^{l} \frac{2}{l !}\left[r^{-1} g_{A_{l}}(t-r)\right]_{A_{l}}, \\
g_{0 j}^{1}= & -\frac{2 \epsilon_{j p q} \S_{p} n_{q}}{r^{2}} \\
& -\sum_{l=2}^{\infty}(-1)^{l} \frac{4 l}{(l+1) !}\left[r^{-1} \epsilon_{j p q} \S_{p A_{l-1}}(t-r)\right]_{, \alpha A_{l-1}} \\
& +\sum_{l=2}^{\infty}(-1)^{l} \frac{4}{l !}\left[r^{-} \dot{\boldsymbol{g}}_{j A}(t-r)\right]_{A_{l-1}}, \\
g_{j k}^{1}= & \delta_{j k}\left(\frac{2 g}{r}+\sum_{l=2}^{\infty}(-1)^{l} \frac{2}{l !}\left[r^{-1} g_{A_{l}}(t-r)\right]_{A_{l}}\right) \\
& +\sum_{l=2}^{\infty}(-1)^{l} \frac{4}{l !}\left[r^{-1} \ddot{g}_{j k A_{l-2}}(t-r)\right]_{, A_{l-2}} \\
& -\sum_{l=2}^{\infty}(-1)^{l} \frac{8 l}{(l+1) !}\left[r^{-1} \epsilon_{p q(j} \dot{\S}_{k) p A_{l-2}}(t-r)\right]_{, \alpha A_{l-2}} .
\end{aligned}
$$

A few comments about this general linearized outgoing-wave solution are in order: (i) The mass monopole $g$ and current dipole $\delta_{j}$ are independent of time by virtue of Eqs. (8.6), (8.10), and (8.11); and $g$ is the total (active gravitational) mass $M$ of the source, while $S_{j}$ is its total angular momentum:

$$
\begin{aligned}
\mathfrak{g} \equiv M & =(\text { mass of source })=\text { constant } \\
\mathcal{S}_{j} \equiv S_{j} & =\text { (angular momentum of source) } \\
& =\text { constant; }
\end{aligned}
$$

cf. Eq. (19.5) of MTW. (ii) In this coordinate system there is no mass dipole moment,

$$
g_{j}=0,
$$

because the origin of coordinates lies at the center of mass of the source, and the coordinates are at rest relative to the center of mass. In other words, the coordinates are "mass centered." (iii) For $l \geqslant 2, g_{A}$ and $\delta_{A_{l}}$ are the mass and current moments of the linearized gravitational waves, as one can see by taking the transverse-traceless ("TT") part of Eq. (8.12c) or $(8.13 \mathrm{c})$ in the radiation zone and comparing with Eq. (4.8): 
$g_{A_{l}}(t)=$ mass $l$-pole moment of radiation field,

$S_{A_{l}}(t)=$ current $l$-pole moment of radiation field.

\section{DYNAMICAL SOURCES IN DE DONDER GAUGE}

\section{A. de Donder equations}

Consider an isolated general relativistic system with arbitrarily strong internal gravity, but with internal motions sufficiently slow that the entire system resides well within its own near zone

$$
\begin{aligned}
L & \equiv(\text { size of system }) \\
& \ll \chi=\left(\begin{array}{l}
\text { characteristic reduced wavelength } \\
\text { of gravitational waves it emits }
\end{array}\right) .
\end{aligned}
$$

We seek the general external gravitational field of such a source, in its near zone

$$
L<r \ll x,
$$

but with the demand that the near-zone field match onto purely outgoing waves (no incoming waves) in the wave zone.

We shall construct the general near-zone field and examine its match to the wave zone, using a de Donder coordinate system which is asymptotically Minkowskii at spatial infinity. Such a coordinate system can obviously be made to cover the entire weak-field region around the source (weak-field near zone plus local wave zone). It will not bother us that the coordinates might go bad in the deep, highly nonlinear interior of the source.

In our de Donder coordinates general relativity theory can be characterized by the following [cf. Sec. II of Thorne and Kovács (1975)]: (i) the gauge condition

$$
\bar{h}_{00,0}=\bar{h}_{0 j, j} ; \quad \bar{h}_{j 0,0}=\bar{h}_{j k, k} ;
$$

(ii) the vacuum field equations

$$
\square \bar{h}_{\alpha \beta} \equiv-\bar{h}_{\alpha \beta, 00}+\bar{h}_{\alpha \beta, j j}=W_{\alpha \beta} ;
$$

(iii) the expression for the "source" in terms of $\bar{h}_{\mu \nu}$ :

$$
W_{\alpha \beta}=-16 \pi(-g) t_{\alpha \beta}^{L L}-\bar{h}_{\alpha \mu, \nu} \bar{h}_{\beta}^{\nu, \mu}+\bar{h}_{\alpha \beta, \mu \nu} \bar{h}^{\mu \nu},
$$

where $t_{\alpha \beta}^{L L}$, the Landau-Lifshitz pseudotensor, and $g$, the determinant of the metric tensor, are both expressible as infinite power series in $\bar{h}_{\alpha \beta}$ [cf. Eqs. (2), (9b), and (9g) of Thorne and Kovács (1975)], and all indices are raised and lowered with the Minkowskii metric; (iv) the asymptotically Minkowskii outgoingwave boundary conditions

$$
\bar{h}_{\alpha \beta}=r^{-1} A_{\alpha \beta}(t-r, \theta, \phi) \text { at } r \rightarrow \infty \text {; }
$$

and (v) equations for computing the metric $g_{\alpha \beta}$ from the gravitational field $\hbar_{\alpha \beta}$,

$$
\begin{gathered}
g^{\alpha \beta} \equiv(-g) g^{\alpha \beta}=\eta^{\alpha \beta}-\bar{h}^{\alpha \beta}, \quad(-g)=\left(-\operatorname{det}\left\|g^{\alpha \beta}\right\|\right)^{-1}, \\
\left\|g_{\alpha \beta}\right\| \equiv\left\|g^{\alpha \beta}\right\|^{-1}
\end{gathered}
$$

[cf. Eqs. (2) and (9d) of Thorne and Kovács (1975)]. Note that the definition $\left\|g_{\alpha \beta}\right\| \equiv\left\|g^{\alpha \beta}\right\|^{-1}$ (Eq. 9.4) is the only place in these equations where indices are not raised and lowered with the Minkowskii metric. For example, the Minkowskii metric is used to obtain $t_{\alpha \beta}^{L L}$ (Eq. 9.3c) from $t_{L L}^{\alpha \beta}$ (Eqs. 2c and $9 \mathrm{~g}$ of Thorne and Kovács, 1975).

\section{B. Nonlinearity expansion}

In solving the field equations and gauge condition (9.3a) and (9.3b), we begin by making a "nonlinearity expansion" of the gravitational field $\bar{h}_{\alpha \beta}$ :

$$
\bar{h}_{\alpha \beta} \equiv \sum_{p=1}^{\infty} G^{p} \gamma_{\alpha \beta}^{p} \text {. }
$$

Here $G$ is Newton's gravitation constant, which has numerical value one in our units, but which serves as a bookkeeping device in the expansion. Note that $\gamma_{\alpha \beta}^{1}$ is the linear part of $\bar{h}_{\alpha \beta} ; \gamma_{\alpha \beta}^{2}$ is the quadratic correction; $\gamma_{\alpha \beta}^{3}$ is the cubic correction, etc.

By inserting (9.5) into the infinite series (9.3c) we obtain a nonlinearity expansion of the source term $W^{\alpha \beta}$. Because $W^{\alpha \beta}$ is of quadratic order and higher in $\bar{h}_{\alpha \beta}$, its nonlinearity expansion begins with $p=2$,

$$
W_{\alpha \beta}=\sum_{p=2}^{\infty} G^{p} w_{\alpha \beta}^{p} ;
$$

$$
w_{\alpha \beta}^{p} \equiv\left[\begin{array}{l}
\text { a sum of terms which each have the form } \\
\gamma_{\mu_{1} \nu_{1}, \sigma}^{a_{1}} \gamma_{\mu_{2} \nu_{2}, \rho}^{a_{2}} \gamma_{\mu_{3} \nu_{3}}^{a_{3}} \cdots \gamma_{\mu_{n} \nu_{n}}^{a_{n}} \text { or } \gamma_{\mu_{1} \nu_{1}, \sigma \rho}^{a_{1}} \gamma_{\mu_{2} \nu_{2}}^{a_{2}} \cdots \gamma_{\mu_{n} \nu_{n}}^{a_{n}}, \\
\text { where } a_{1}+\cdots+a_{n}=p \text { and where } n \geqslant 2 \text { so } a_{j} \leqslant p-1 \text { for all } j
\end{array}\right] .
$$

The gauge condition and field equations (9.3a) and (9.3b) must now be satisfied order-by-order in the nonlinearity expansion

$$
\begin{aligned}
& \gamma_{00,0}^{p}=\gamma_{0 j, j}^{p} ; \gamma_{j 0,0}^{p}=\gamma_{j k, k}^{p} ; \\
& \square \gamma_{\alpha \beta}^{p}=w_{\alpha \beta}^{p} .
\end{aligned}
$$

Because $w_{\alpha \beta}^{p}$ is constructed only from $\gamma^{\alpha}$ with $q \leqslant p-1$, these equations can be solved first for $\gamma^{1}$ ignoring all higher-order $\gamma^{\prime}$; then for $\gamma^{2}$ which is generated by $\gamma^{1}$ via the field equations (9.7b); then for $\gamma^{3}$, which is generated by $\gamma^{1}$ and $\gamma^{2}$ via the field equations; etc. Once all the $\gamma^{p}$ are known up to some desired order, one can calculate from Eqs. (9.4) the corresponding terms in a nonlinearity expansion of the metric

$$
g_{\alpha \beta}=\eta_{\alpha \beta}+\sum_{p=1}^{\infty} G^{p} g_{\alpha \beta}^{p} .
$$

It seems almost certain to me (but I do not claim to have proved it) that this procedure will generate a nonlinearity series (9.5) that converges throughout the weak-field near zone, the local wave zone, and the region linking them [see Eq. (3.2) for terminology]. 
However, one cannot expect convergence either in the strong-field region where nonlinearities are huge, or in the distant wave zone, where nonlinearities will produce large phase shifts of the waves

$$
\Delta \psi \sim(M / x) \ln (r / x) \gtrsim 1
$$

[Eq. (3.4)].

\section{Linear part of the gravitational field}

The linear part $\gamma_{\mu \nu}^{1}$ of the general nonlinear field $\bar{h}_{\mu \nu}$ satisfies the field equations and gauge conditions of Linearized Theory [compare Eqs. (9.7) for $p=1$ with Eqs. (8.3)]. Consequently, with an appropriate specialization of the de Donder coordinates [that nonlinear generalization of Eqs. (8.7b), (8.8b), and (8.9) which leaves the de Donder gauge condition (9.3a) unaffected to all orders], the most general $\gamma_{\mu_{\nu}}^{1}$ can be put into the same form (8.12) as our general linearized-theory field.

Note that this general $\gamma_{\mu \nu}^{1}$ is determined by a constant mass $\mathscr{S}=M$, a constant angular momentum $\mathcal{S}_{j}=S_{j}$, and two sets of time-dependent multipole moments $\mathfrak{g}_{A_{l}}(t)$ and $S_{A_{l}}(t)$ with $l \geqslant 2$.

\section{Multiparameter expansion of the near-zone gravitational field}

Thus far our analysis has been valid throughout the local wave zone, the weak-field near zone, and the transition region which links them. We now restrict attention to the weak-field near zone and initiate a study of the nonlinear corrections $\gamma_{\alpha \beta}^{p}(p \geqslant 2)$ to $\bar{h}_{\alpha \beta}$ there.

For this purpose we introduce three length scales $M$, $R, \chi$ which characterize the near-zone field: $M$ is the mass of the source at linear order

$$
M \equiv \boldsymbol{g} ;
$$

$R$ is a length scale characterizing the nonspherical deformations of the source's gravitational field

$$
\begin{aligned}
& R \equiv\left(\begin{array}{l}
\text { maximum over all } l \geqslant 1 \text { and } \\
\text { over all times of interest }
\end{array}\right) \\
& \quad \text { of }\left\{\left|g_{A_{l}} / M\right|^{1 / t},\left|\delta_{A_{l}} / M\right|^{1 / l}\right\} ;
\end{aligned}
$$

and $x$ is the characteristic time scale on which the multipole moments change

$$
\frac{1}{\bar{x}} \equiv\left(\begin{array}{l}
\text { typical value } \\
\text { over all } l \geqslant 2
\end{array}\right) \text { of }\left\{\frac{\dot{g}_{A_{l}}}{\dot{g}_{A_{l}}}, \frac{\dot{S}_{A_{l}}}{\dot{S}_{A_{l}}}\right\} \text {. }
$$

For a realistic source of size $L$ it is reasonable to expect $R \leqslant L$; cf. Eqs. (5.19); and in general the definitions (9.10) guarantee

$$
\begin{aligned}
& \left|g_{A_{l}}\right| \lesssim M R^{l}, \quad\left|\delta_{A_{l}}\right| \lesssim M R^{l}, \\
& \left|\dot{g}_{A_{l}}\right| \lesssim M R^{l} / \chi, \quad\left|\dot{\delta}_{A_{l}}\right| \leqslant M R^{l} / \chi .
\end{aligned}
$$

(If we wished, we could introduce a "rotational velocity parameter" $v$ to distinguish current moments from mass moments; but such a parameter would make the discussion overly complicated.) Our slow-motion assumption (source deep within its own near zone) re- quires

$$
x \gg M, \quad x \gg L \gtrsim R .
$$

The "weak-field near zone" [Eq. (3.2)] is the region

$$
x \gg r, \quad r \gg M, r>L \gtrsim R \text {. }
$$

We regard the multipole moments $\mathcal{g}_{A_{l}}(t)$ and $\delta_{A_{l}}(t)$ as freely specifiable so that one can scale up or down each of the parameters $M, R$, and $x$ while leaving the others unchanged. In such scaling

$$
\begin{aligned}
& \mathscr{g}_{A_{l}} \text { and } \delta_{A_{l}} \text { scale as } M R^{l} ; \\
& \partial_{t} \mathscr{g}_{A_{l}} \text { and } \partial_{t} \delta_{A_{l}} \text { scale as } M R^{l} / x .
\end{aligned}
$$

We expand $\bar{h}_{\mu \nu}, W_{\mu \nu}$, and $g_{\mu \nu}$ in infinite series of terms, each term of which scales (at fixed $r$ ) as a definite power of $M$, of $R$, and of $1 / x$; and each of which has a definite spherical-harmonic order $l$ :

$$
\begin{aligned}
& \bar{h}_{\mu \nu}=\sum_{p, n, u, l} \gamma_{\mu \nu}^{p m u t}, W_{\mu \nu}=\sum_{p, n, u, l} w_{\mu \nu}^{p n u l}, \\
& g_{\mu \nu}=\sum_{p, n, u, l} g_{\mu \nu}^{p m u l} .
\end{aligned}
$$

Here

$\gamma_{\mu \nu}^{p n u l}$ scales as $M^{b} R^{n}(1 / \chi)^{u}$ and has spherical-harmonic order $l$;

and similarly for $w_{\mu \nu}^{p n u l}, g_{\mu \nu}^{p n u l}$. These expansions should converge in the weak-field near zone, and only there, since they actually turn out to be expansions in powers of $g_{A l} / r^{l+1}, S_{A l} / r^{l+1}$, and $r / x$-or, equivalently, in powers of $M / r, R / r$, and $r / x$; and all of these quantities are $\ll 1$ in the weak-field near zone [except extremely near the source; Eqs. (9.13)], but some of them are $z 1$ in any other vacuum region of spacetime.

In addition to the quantities $\gamma_{\mu \nu}^{p n u l}, w_{\mu \nu}^{p n u l}, g_{\mu \nu}^{p m u l}$, which have pure scale properties, we define the sum over one or more of the parameters in these quantities, and we denote a summed parameter by a dot:

$$
\gamma_{\mu \nu}^{p n u \cdot} \equiv \sum_{l} \gamma_{\mu \nu}^{p m u l} ; g_{\mu \nu}^{\cdot u \cdot}=\sum_{p, n, l} g_{\mu \nu}^{p n u l} ; \text { etc. }
$$

Since all the multipole moments scale as $M$, the $\gamma_{\mu \nu}^{1}$ of our nonlinearity expansion also scales as $M$, and-as one can see from Eqs. (9.6), (9.7) $-\gamma_{\mu \nu}^{p}$ scales as $M^{p}$. Thus the $\gamma_{\mu \nu}^{p}$ of our nonlinearity expansion (Sec. IX.B) is equal to $\gamma_{\mu \nu}^{p \cdots}$; and similarly for $w$ and $g$ :

$$
\gamma_{\mu \nu}^{p}=\gamma_{\mu \nu}^{p \cdots}, \quad w_{\mu \nu}^{p}=w_{\mu \nu}^{p \cdots}, g_{\mu \nu}^{p}=g_{\mu \nu}^{p \cdots} .
$$

By comparing Eqs. (8.12), (2.53), (9.14), and (9.16), we can read off the various subpieces of the linearorder field $\gamma_{\mu \nu}^{1}$. For example, the only nonzero pieces of $\gamma_{00}^{1}$ are

$$
\begin{aligned}
& \gamma_{00}^{1000}=4 g / r ; \\
& \gamma_{00}^{11(2 k) l} \\
& \quad=-\frac{4(2 l+1) ! ! r^{2 k-l-1}}{l ! 2^{k} k !(-1-2 l)(1-2 l) \cdots(2 k-1-2 l)}{ }^{(2 k)} g_{A_{l}}(t) N_{A_{l}} \\
& \gamma_{00}^{1 l(2 k+1) l}=-\frac{(-1)^{l} 4 r^{2 k-l}}{l ! 2^{k-l}(k-l) !(2 k+1) ! !}{ }^{(2 k+1)} g_{A_{l}}(t) N_{A_{l}} \\
& \text { for } l \geqslant 2, k \geqslant 0 ; \quad(9.19 \mathrm{~b})
\end{aligned}
$$


Here a prefix superscript in parentheses means differentiation with respect to time, ${ }^{(2 k)} g_{A_{l}} \equiv\left(\partial_{t}\right)^{2 k} g_{A_{l}}$, and $N_{A_{1}}$ means $n_{a_{1}} n_{a_{2}} \cdots n_{a_{l}}$; see Sec. I.C and Eq. (2.54). It is straightforward to verify that the only nonzero parts of $\gamma_{\mu \nu}^{1}$ are [Eq. (8.12)]

$$
\begin{aligned}
& \gamma_{00}^{1000} ; \quad \gamma_{00}^{1 l(2 k) l} \text { for } l \geqslant 2, k \geqslant 0 ; \\
& \gamma_{00}^{1 l(2 k+1) l} \text { for } k \geqslant l \geqslant 2 ; \\
& \gamma_{0 j}^{1101} ; \gamma_{0 j}^{1 l l k l .} \text { for } l \geqslant 2, k \geqslant 0 ; \\
& \gamma_{i j}^{1 l k l} \text { for } l \geqslant 2, k \geqslant 1 .
\end{aligned}
$$

\section{E. Multiparameter expansion of the near-zone gauge conditions and field equations}

The various $\gamma_{\mu \nu}^{p m l}$ are coupled together by the field equations, the de Donder gauge condition, and the match of the near-zone solution onto outgoing waves in the local wave zone. Because a time derivative $\partial_{t}$ increases by 1 the $(1 / x)$ order of a quantity, and because divergences and Laplacians leave unchanged the sphericalharmonic order of a quantity, the gauge conditions and field equations (9.7) say

$$
\begin{aligned}
& \gamma_{0 j, j}^{p m u l}=\gamma_{00,0}^{p n(u-1) t} ; \gamma_{j k, k}^{p m u l}=\gamma_{j 0,0}^{p n(u-1) t} ; \\
& \nabla^{2} \gamma_{\mu \nu}^{p n u t}=\gamma_{\mu \nu, 00}^{p n(u-2) l}+w_{\mu \nu}^{p n u l} .
\end{aligned}
$$

The source term $w_{\mu \nu}^{p \text { nul }}$ has the form

$$
w_{\mu \nu}^{p m u l}=\left[\begin{array}{l}
\text { a sum of terms, each of which has the form } \\
\left(\prod_{j=1}^{N} \gamma_{\alpha \beta}^{p_{j} n_{j} u_{j} l_{j}}\right)^{l-\text { order part }}, \text { with } v \text { time derivatives }(v \leqslant 2) \text { and } 2-v \text { spatial } \\
\text { derivatives interspersed among the terms of the product; and with } \\
\sum p_{j}=p \geqslant 2, \sum n_{j}=n, v+\sum u_{j}=u, \sum l_{j} \geqslant l, \min \left|l_{1} \pm l_{2}{ }^{\prime} \cdots \pm l_{N}\right| \leqslant l
\end{array}\right] ;
$$

cf. Eqs. (9.3c), (9.15), (9.16), and the "angular momentum coupling rules." Because $W^{\mu \nu}{ }_{, \nu}=0$ and because the decomposition (9.15) of $W^{\mu \nu}$ is unique, $w_{\mu \nu}^{p m u l}$ satisfies the necessary conditions for simultaneous integration of (9.21a) and (9.21b):

$$
w_{0 j, j}^{p n u l}=w_{00,0}^{p n(u-1) l} ; \quad w_{j k, k}^{p n u l}=w_{j 0,0}^{p n(u-1) t} .
$$

The nonlinear terms $\gamma_{\mu \nu}^{p \text { nut }}$ with $p \geqslant 2$ can be calculated one after another from the known linear terms and from already-calculated nonlinear terms by solving Eqs.

(9.21). At each stage in the solution the right-hand side of $(9.21 \mathrm{~b})$ is known explicitly, and the higher-order left-hand side must be calculated subject to the consistency conditions (9.21a). For example, it is straightforward to find from Eqs. (9.3c), (9.19), and (9.20) that

$$
w_{00}^{2000}=\frac{7}{8} \gamma_{00, j}^{1000} \gamma_{00, j}^{1000}=14 g^{2} / r^{4} \text {. }
$$

Inserting this into the field equations (9.21b) one obtains

$$
\gamma_{00}^{2000}=7 g^{2} / r^{2} \text {, }
$$

which presents no problems in (9.21a) since $g$ is time independent.

At each step in this procedure, one calculates $\gamma_{00}^{\text {pnut }}$ from its known Laplacian; or one calculates $\gamma_{\mu j}^{p m u l}$ from its known Laplacian and divergence. In the calculation one must use care to discern whether to add on a Laplacian-free and divergence-free piece (homogeneous solution). One never adds on such a piece with radial behavior $r^{-\left(l^{\prime}+1\right)}, l^{\prime} \geqslant 0$, since such a piece could perfectly well have been included in the linear part of the field $\gamma_{\mu \nu}^{1}$. However, one is sometimes forced to add on a homogeneous solution with radial behavior $r^{l^{\prime}}$ in order to guarantee that the near-zone field matches onto outgoing waves in the local wave zone. [The term $\gamma_{00}^{1 l(2 l+1) t}$ in $(9.19 \mathrm{c})$ is an example; it is not generated by the near-zone field equations or gauge conditions; and it would change sign if one were to switch from an outgoing -wave boundary condition to ingoing waves.] The homogeneous part which one adds onto $\gamma_{\mu \nu}^{p h u l}$ must scale as $M^{p} L^{n} \chi^{-u}$, must be dimensionless, must have vanishing Laplacian, and must have spherical-harmonic order $l$. Therefore, unless the homogeneous piece is a "tail term" (see Appendix), its radial dependence must be $r^{-p-n+u}=r^{l^{\prime}}$, where $l^{\prime} \geqslant 0, l^{\prime}=l$ if $\mu$ and $\nu$ are both temporal, $l-1 \leqslant l^{\prime} \leqslant l+1$ if only one of $\mu$ and $\nu$ is temporal, and $l-2 \leqslant l^{\prime} \leqslant l+2$ if $\mu$ and $\nu$ are both spatial. Thus

$\gamma_{\mu \nu}^{p \text { nul }}$ can contain a homogeneous matching-generated piece only if $u \geqslant p+n+l^{\prime}$, $l^{\prime}=\max [0, l-($ number of spatial indices on $\gamma)]$.

\section{F. Structure of the general near-zone term in the multiparameter expansion}

The general term $\gamma_{\mu \nu}^{p m i l}$ generated by the above procedure must be a sum of quantities which each have the following form:

$$
\gamma_{\mu \nu}^{p n u l}=\left[\left(u_{0}\right)\left(\prod_{j=1}^{p}{ }^{\left(u_{j}\right)} \mathfrak{T}^{l_{j} \pi_{j}}\right)\right]{ }^{l \pi} r^{-p-n+u} \sum_{k=0}^{K} C_{k}\left(\ln \frac{r}{R}\right)^{k}
$$

(Matching-generated tail terms are the sole exception; see Appendix.) Here ${ }^{\left(u_{0}\right)}(\ldots)$ means the $u_{0}$ th time derivative of the quantity in parentheses, and $u_{0}$ can be negative; $\mathfrak{N}^{l j^{\pi} j}$ is the multipole moment of the linear part of the field with order $l_{j}$ and parity $\pi_{j}$ :

$$
\begin{aligned}
& \mathfrak{M}^{l_{j} \pi_{j}}=g_{A_{l_{j}}} \text { if } \pi_{j}=(-1)^{l_{j}}, \\
& \mathfrak{M}^{l_{j} \pi_{j}}=\epsilon_{i p q} S_{p A_{l_{j}-1}} n_{q} \text { if } \pi_{j}=(-1)^{l_{j}^{+1}} ;
\end{aligned}
$$

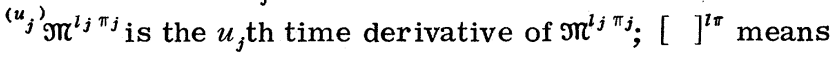


that the components of the $p$ multipole moments are coupled together to give a spherical harmonic of order $l$ and parity $\pi$; and $C_{k}$ and $R$ are constants.

There are $p$ moments $\mathfrak{T}$ present in (9.25) so as to guarantee that the right-hand side scales as $M^{p}$.

Scaling in $L$ and $x$ requires

$$
\sum_{j=1}^{p} l_{j}=n, \quad \sum_{j=0}^{p} u_{j}=u,
$$

[cf. Eqs. (9.14) and (9.16)]. The laws of angular momentum coupling require

$$
\sum_{j=1}^{p} l_{j}=n \geqslant l, \quad \min \left|\sum_{j=1}^{p} \pm l_{j}\right| \leqslant l .
$$

The radial dependence of Eq. (9.25) is forced on us by the demand that $\gamma_{k: \nu}$ be dimensionless. A logarithmic term, $\ln (r / R)$, is created whenever one inverts the field equation (9.21b) with a source term of the form (Laplacian-free function) $\times r^{-2}$; for example,

$$
\nabla^{2} \gamma_{00}^{24104}=\left[{ }^{(4)} g_{a_{1} a_{2}}{ }^{(6)} g_{a_{3} a_{4}}\right]^{\mathrm{STF}} N_{A_{4}} \gamma^{2}
$$

is inverted to give

$$
\gamma_{00}^{24104}=\frac{1}{9}\left[{ }^{(4)} \boldsymbol{g}_{a_{1} a_{\dot{2}}}{ }^{(6)} \boldsymbol{g}_{a_{3} a_{4}}\right]^{\mathrm{STF}} N_{A_{4}} r^{4} \ln (r / R) .
$$

One can show that the maximum number $K$ of logarithms in Eq. (9.25) is bounded by

$$
K \leqslant p-1 \text {. }
$$

The proof is by induction: There are no logarithms present at linear order [Eqs. (8.12) and (2.53b)], so $(9.26 \mathrm{~d})$ is true for $p=1$. Assume it true for $1,2, \ldots, p-1$. Then the maximum number of logarithms in $w_{\mu \nu}^{\text {prul }}$, which has the form $(9.22 \mathrm{a})$, is

$$
\sum_{j=1}^{N} K_{j} \leqslant \sum_{j=1}^{N}\left(t_{j}-1\right)=p-N \leqslant p-2 .
$$

Imagine solving the field equations (9.21b) for $\gamma_{\mu \nu}^{p m u} l, l^{\prime}$ $\equiv$ (that part of $\gamma_{\mu \nu}^{p n u l}$ with "pure-orbital" spherical-harmonic dependence $Y^{l^{\prime} m}$ for $\mu \nu$ both temporal, or $\mathbf{Y}^{l^{\prime}, l m}$ (Sec. II.D) for one of $\mu \nu$ spatial, or $\mathbf{T}^{\lambda l^{\prime}, l m}$ (Sec. II.E) for $\mu \nu$ both spatial). These field equations, with $p, n, l, l^{\prime}$ fixed, can be solved in two sequences: the first for $u=\ldots,-2,0,2,4, \ldots$; the second for $u=\ldots,-1,1,3,5, \ldots$. Along each sequence, precisely once (at $u-p-n=-l^{\prime}-1$ or at $u-p-n=l^{\prime}$; cf. Eq. [2.42]) we will encounter a source term of the form

$$
\begin{aligned}
& \gamma_{\mu \nu, 00}^{p p(u-2) l, l^{\prime}}+w_{\mu \nu}^{p n u l, l^{\prime}}= \\
& =(\text { Laplacian-free function }) \times r^{-2} \sum_{k=0}^{p-2} C_{k}[\ln (r / R)]^{k} .
\end{aligned}
$$

Before that source term is encountered, the $\gamma_{\mu \nu}^{p n u l, l^{\prime}}$ will have at most $p-2$ logarithms. That special source term, like (9.27a), will generate one new logarithmgiving at most $p-1$ logarithms in any of the $\gamma_{\mu \nu}^{p m u l, l^{\prime}}$. Thus $K \leqslant p-1$. QED

Since the parity of a quantity [Eq. (2.17)] is not changed by the linear manipulations involved in computing $\gamma_{\mu \nu}^{p m u l}$ (taking the time derivative, divergence, Laplacian, or inverse Laplacian; matching out into the wave zone and then back in, selecting out a part with specific spherical harmonic order); and since the con- struction of the "source terms" $w_{\mu \nu}$ from the $\gamma$ 's involves only simple products without any parity changing $\dot{\epsilon}_{i j k}$ 's [cf. Eq. (9.3c)]; and since the parity of a product is the product of the parities, the coupling of multipoles in Eq. (9.25) must produce a harmonic with overall parity

$$
\pi=\pi_{1} \pi_{2} \cdots \pi_{p} \text {. }
$$

There is a minimum value which each integer $p, n, u$, $l$ must have in Eq. (9.25) in order for $\gamma_{\mu \nu}^{p n u l}$ to be nonzero. Obviously $p>0$ [otherwise there will be no $s$ 's or g's in Eq. (9.25)]; $n \geqslant 0$ [since $n=\sum l_{j}$ and sphericalharmonic indices $l_{j}$ can never be negative]; $l \geqslant 0$ [same reason]; and $u \geqslant 0$ [this one proves by induction: it is true for $\gamma_{\mu \nu}^{1}$ [Eq. (9.20)]; if it is true for all $p$ up to $p_{0}-1$ then $w^{p_{0} n u l}$ is zero unless $u \geqslant 0$ [Eq. (9.22)], so the inhomogeneous solutions to Eq. (9.21) for $\gamma^{p_{0}}{ }^{n u l}$ vanish unless $u \geqslant 0,{ }^{3}$ and Eq. (9.24) guarantees that the "non-tail" homogeneous terms produced by matching have $u \geqslant p_{0}+n$ $>0$; thus $u \geqslant 0$ for all nonzero $\left.\gamma^{p_{0} n u l}\right]$. In summary:

$$
\gamma_{\mu \nu}^{p n u l}=0 \text { unless } p>0, n \geqslant 0, u \geqslant 0, l \geqslant 0 .
$$

By noting how $g_{\mu \nu}^{p n u l}$ is constructed algebraically from the $\gamma_{\alpha \beta}^{p^{\prime} n^{\prime} u^{\prime} l^{\prime}}$ [Eqs. (9.4) and (9.15)], and by then applying the same considerations as above to $g_{\mu \nu}^{p n u t}$, one concludes that the "non-tail" $g_{\mu \nu}^{p n u}$ has the same structure [Eqs. (9.25) and (9.26)] as $\gamma_{\mu \nu}^{\text {pnut }}$ : It is a sum of terms each having the form

$$
g_{\mu \nu}^{p n u l}=\left[{ }^{\left(u_{0}\right)}\left(\prod_{j=1}^{p}{ }^{\left(u_{j}\right)} \mathfrak{M}^{{ }^{l}{ }_{j} \pi_{j}}\right)\right]^{2 \pi} r^{-p-n+u} \sum_{k=0}^{K} C_{k}\left[\ln \left(\frac{\gamma}{R}\right)\right]^{k},
$$

where

$$
\text { all of the properties (9.26) hold for } g_{\mu \nu}^{p n u l} \text {. }
$$

\section{G. Secular changes of mass, linear momentum, and angular momentum}

It is instructive to see how the above formalism produces a secular change in the mass, linear momentum, and angular momentum of the source which is equal and opposite to the mass, linear momentum, and angular momentum carried off by the waves. (That such a change must occur is proved, e.g., in Chap. 20 of MTW.) In the term $w_{0 j}^{2(2 l)(2 l+2) 0}$ there is a piece

$$
\begin{aligned}
w_{0 j}^{2(2 l)(2 l+2) 0}= & \text { const. } \times\left[\gamma_{00,0}^{1 l 0 l} \gamma_{00, j}^{1 l(2 l+1) l}\right]^{0} \\
= & \text { const. } \times r^{-2} n_{j}{ }^{(1)} g_{A_{l}}{ }^{(2 l+1)} g_{A_{l}} \\
= & \text { const. } \times r^{-2} n_{j}{ }^{(l+1)} g_{A_{l}}{ }^{(l+1)} g_{A_{l}} \\
& +\left[\begin{array}{l}
\text { a perfect time derivative of a } \\
\text { quantity quadratic in time } \\
\text { derivatives of } g_{A_{l}}
\end{array}\right]
\end{aligned}
$$

[cf. Eqs. (9.22a) and (9.19)]. This piece describes, in the near zone, an outward flux of energy associated

\footnotetext{
${ }^{3)} \mathrm{A}$ delicacy occurs in situations like Eq. $(9.29 \mathrm{c})$, where a Laplacian-free $\gamma_{00}^{p n(u-1) l}$ or $\gamma_{0 j}^{p n(u-1) l}$ is generated, through the gauge conditions, by $\gamma_{0 j}^{p_{0}^{n u l}}$ or $\gamma_{j k}^{p \text { nul }}$. However, this "lowering of $u$ by one" occurs only when the original $u$ is greater than zero; so it presents no problems.
} 
with the mass $l$-pole part of the radiation field. By way of the field equation $(9.21 \mathrm{~b})$ it generates

$$
\begin{aligned}
\gamma_{0 j}^{2(2 l)(2 l+2) 0}= & \text { const. } \times_{n_{j}}{ }^{(l+1)} g_{A_{l}}{ }^{(l+1)} g_{A_{l}} \\
& +(\text { perfect time derivative }) ;
\end{aligned}
$$

and then, by way of the gauge condition (9.21a), this generates

$$
\begin{aligned}
\gamma_{00}^{2(2 l)(2 l+1) 0}= & (\text { const. } / \gamma) \times{ }^{(-1)}\left({ }^{(l+1)} g_{A_{l}}(l+1) g_{A_{l}}\right) \\
& +(\text { oscillatory quantity) } .
\end{aligned}
$$

This term has the same form as the linear mass monopole part of the field, $4 \mathrm{~g} / \mathrm{r}$. When averaged over several characteristic time scales $\pi$, it and other similar terms represent a tiny, secular correction to the mass of the source. The tiny, secular change of linear momentum shows up as an analogous term in the (originally zero) $1 / r^{2}$ mass dipole part of $\gamma_{00}$; and the tiny secular change of angular momentum shows up as an analogous term in the $1 / \gamma^{2}$ current-dipole part of $\gamma_{0 j}$.

This manner in which our formalism generates the secular, "radiation-reaction" terms is analogous to the manner in which the Bondi-van der Burg-Metzner (1962) formalism does so. For insight, including a "nonlinearity expansion" similar to the one given here, see Bonnor and Rotenburg (1966).

\section{H. Radiation field}

Turn attention now to the "transition region" of space which includes the outer reaches of the near zone and the inner parts of the local-wave zone:

$$
\begin{aligned}
& \alpha_{1} \pi<r<\pi / \alpha_{2}, \alpha_{1} \text { and } \alpha_{2} \text { constants with } \alpha_{i} \ll 1, \\
& \text { but } \quad \alpha_{1} \pi \gg \max (M, L, R), \\
& x / \alpha_{2} \ll r_{0}[\mathrm{Eq} .(3.4)] .
\end{aligned}
$$

Throughout this transition region gravity is so weak, $\left|\hbar_{\mu \nu}\right| \ll 1$, that we shall ignore nonlinearities in the field equations. Consequently, in a suitably chosen gauge $\bar{h}_{\mu \nu}$ will have the form (8.12) characteristic of the general outgoing-wave solution of the linearized field equations

$$
\begin{aligned}
& \hbar_{00}=\frac{4 g^{\mathrm{rad}}}{r}+\sum_{i=2}^{\infty}(-1)^{l} \frac{4}{l !}\left[r^{-1} \mathcal{g}_{A_{l}}^{\mathrm{rad}}(t-r)\right]_{A_{l}}, \\
& \bar{h}_{0 j}=\frac{-2 \epsilon_{j p} S_{p}^{\text {ad }} n}{r^{2}} \\
& -\sum_{l=2}^{\infty}(-1)^{l} \frac{4 l}{(l+1) !}\left[r^{-1} \epsilon_{j p q} \mathcal{S}_{p A_{l-1}}^{\mathrm{rad}}(t-r)\right]_{, A_{l-1}} \\
& +\sum_{l=2}^{\infty}(-1)^{l} \frac{4}{l !}\left[r^{-1} \dot{\boldsymbol{g}}_{j A_{l-1}}^{\mathrm{rad}}(t-r)\right]_{A_{l-1}}, \\
& \hbar_{j k}=\sum_{l=2}^{\infty}(-1)^{\imath} \frac{4}{l !}\left[r^{-1} \ddot{\boldsymbol{g}}_{j k A_{l-2}}^{\mathrm{rad}}(t-r)\right]_{A_{l-2}} \\
& -\sum_{l=2}^{\infty}(-1)^{l} \frac{8 l}{(l+1) !}\left[r^{-1} \epsilon_{p q(j} \dot{\mathbf{S}}_{k)_{p A}{ }_{l-2}}^{\mathrm{rad}}(t-r)\right]_{,{ }_{q A}{ }_{l-2}} \\
& \text { throughout transition region. (9.31c) }
\end{aligned}
$$

In the local wave zone itself, $r \gg \lambda$, the radiation field [obtained by evaluating the derivatives in Eq. (9.31c) with the help of Eq. (2.53) and by keeping only terms of order $1 / r]$ is

$$
\begin{aligned}
\bar{h}_{j k}^{\mathrm{TT}}= & {\left[\sum_{l=2}^{\infty} \frac{4}{l !} r^{-1(l)} \boldsymbol{g}_{j k A}^{\mathrm{rad}}(t-r) N_{A_{l-2}}\right.} \\
& \left.+\sum_{l=2}^{\infty} \frac{8 l}{(l+1) !} r^{-1} \epsilon_{p q(j}{ }^{(l)} \boldsymbol{s}_{k)_{p A}{ }_{l-2}}^{\mathrm{rad}}(t-r) n_{q} N_{A_{l-2}}\right]^{\mathrm{T} \mathrm{T}} .
\end{aligned}
$$

Comparison with Eqs. (4.8) shows that $g_{A_{l}}^{\mathrm{rad}}$ and $\mathcal{S}_{A_{l}}^{\mathrm{rad}}$ are the mass and current moments of the radiation fieldhence the superscripts "rad."

The relationship between the radiation-field moments $\mathcal{g}_{A_{l}}^{\mathrm{rad}}, \boldsymbol{S}_{A_{l}}^{\mathrm{rad}}$ and the near-zone moments $\boldsymbol{g}_{A_{l}}, \boldsymbol{S}_{A_{l}}$ can be inferred by comparing the transition-region solution (9.31) for $\bar{h}_{\mu \nu}$ with the non-tail near-zone solution (9.5), (8.12) at radii in the domain of overlap $\alpha_{1} x \lessgtr r \leqslant x$ (method of "matched asymptotic expansions"):

(l) $g_{A_{l}}^{\mathrm{rad}}={ }^{(l)} g_{A_{l}}+\left[\begin{array}{l}\text { a nonlinear correction of magnitude } \\ \text { equal to the } l \text {-pole, electric-parity } \\ \text { part of } x \sum_{p=2}^{\infty} \gamma_{j k}^{p} \text { evaluated at } r \simeq \star\end{array}\right]$

${ }^{(l)} S_{A_{l}}^{\text {rad }}={ }^{(2)} S_{A_{l}}+\left[\begin{array}{l}\text { a nonlinear correction of magnitude } \\ \text { equal to the } l \text {-pole, magnetic-parity } \\ \text { part of } \pi \sum_{p=2}^{\infty} \gamma \gamma_{j k}^{p} \text { evaluated at } r \simeq \pi\end{array}\right]$.

Comparison with Eqs. (9.25) and (9.26) then shows that near-zone the nonlinearities contribute the following amounts to the multipoles of the radiation field:

$$
\begin{array}{r}
\delta \mathfrak{M}^{l \pi}=\mathfrak{M}_{\mathrm{rad}}^{l \pi}-\mathfrak{N}^{l \pi}=\text { a sum of terms with magnitudes } \\
\qquad \pi^{1+l-p-\Sigma l_{j}}\left(\prod_{j=1}^{p} \mathfrak{M}^{l_{j} \pi_{j}}\right]^{l \pi}\left[\ln \left[\frac{\pi}{R}\right]\right]^{K}
\end{array}
$$

where

$$
p \geqslant 2, \quad \sum_{j=1}^{p} l_{j} \geqslant l, K \leqslant p-1 .
$$

It is straightforward to verify, from Eqs. (9.33), (9.26a), and (9.11) that those radiative moments which dominate the radiation field - the ones with the largest values of $\left.\right|^{(l)} \mathrm{M}^{l \pi} \mid$-will contain negligible nonlinear corrections :

$$
\left|\delta \mathfrak{M}^{l \pi}\right| /\left|\mathfrak{M}^{l \pi}\right| \lesssim(M / \pi) \ln (\pi / R) .
$$

This means that throughout the local wave zone the radiation field is equal to the linear-order field [Eq. $(8.12 \mathrm{c})]$ to within fractional errors $\lesssim(M / \chi) \ln (\chi / R)$ :

$$
\hbar_{j k}^{\mathrm{TT}}=\left(\gamma_{j k}^{1}\right)^{\mathrm{TT}} \times\{1+O[(M / \pi) \ln (\pi / R)]\}
$$

in local wave zone.

[One can show - but this is not the appropriate place to do so-that the only radially cumulative nonlinear effects in the local wave zone which exceed $M / \chi$, are trivial phase shifts (Sec. III) and gauge changes. Among the nonlinear local-wave-zone effects of magnitude $\lesssim M / \lambda$ are the tails of the waves; see Appendix for discussion.]

\section{Summary}

The above formalism shows explicitly, in de Donder gauge, how the full nonlinear near-zone external grav- 
itational field of the source is determined by its timedependent multipole moments, together with the outgoing-wave boundary condition. That the multipole moments plus outgoing-wave condition do fully determine the field has been known for a long time [Janis and Newman (1965) Bonnor and Rotenburg (1966)]. I have gone into this great detail on a well-known subject in order to obtain the form (9.28) of the general non-tail term in the expansion (9.15) of the metric, and in order to relate the near-zone field to the wave-zone field [Eq. $(9.35)]$. We will need these relations below.

\section{STATIONARY SOURCES IN DE DONDER GAUGE}

Consider next a stationary, isolated system residing in asymptotically flat spacetime. Introduce coordinates $\left\{x^{\alpha}\right\}$ such that $\partial / \partial x^{0}$ is the Killing vector field which generates the stationary time translations, and such that the metric coefficients are asymptotically Lorentz

$$
g_{\alpha \beta}=\eta_{\alpha \beta}+O(1 / r) \text {. }
$$

Then perform a coordinate transformation of the form

$$
x_{\text {ne }}^{\alpha}=x_{\text {old }}^{\alpha}+f^{\alpha}\left(x_{\mathrm{old}}^{j}\right) ; f^{\alpha}=\text { constant }+O(1 / r) .
$$

This keeps $\partial / \partial x^{0}=$ (Killing vector) and maintains the asymptotically flat form (10.1) of the metric. Adjust the four functions $f^{\alpha}\left(x_{\mathrm{old}}^{j}\right)$ so that the metric in the new coordinate system satisfies the stationary de Donder gauge conditions

$$
g^{\alpha_{j}}{ }_{, j}=0 \text {, }
$$

where $g^{\alpha \beta}$ is the metric density [Eq. (9.4)]. In this stationary de Donder coordinate system the metric is independent of $x^{0}$.

Now use the same procedure as was used for the dynamical case (Sec IX) to construct the general external gravitational field of such a stationary, isolated system. The result will be the same as in Sec. IX [Eqs. (9.15), (8.13), and (9.28)] with these exceptions: (i) Because of stationarity, the general term (9.28) in the solution must have

$$
u=u_{0}=u_{1}=\cdots=u_{p}=0 .
$$

(ii) Because of stationarity $w_{\mu \nu}^{\text {pnol, } l^{\prime}}$ always has radial dependence $r^{-(p+n+2)}$ with $p+n \geqslant p+l^{\prime} \geqslant l^{\prime}+2$. This guarantees that situations like (9.27), which produce logarithmic terms, never arise; i.e., the logarithmic terms are completely absent in Eq. (9.28); i.e.,

$$
K=0 \text {. }
$$

(iii) Because of stationarity there are no tail terms.

These considerations show that the general stationary metric can be written in the form

$$
g_{\mu \nu}=\sum_{p n l} g_{\mu \nu}^{p n l}
$$

where

$$
g_{\mu \nu}^{1}=\sum_{n, l} g_{\mu \nu}^{1 n \imath}=\left(\begin{array}{l}
\text { expression (8.13) with } g_{A} \\
\text { and } S_{A_{l}} \text { independent of } t-r
\end{array}\right) ;
$$

and where the nonlinear corrections $g_{\mu \nu}^{p n l}$ are sums of terms which each have the form

$$
g_{\mu \nu}^{p n l}=\left[\prod_{j=1}^{p} \Re^{l_{j} \pi_{j}}\right]^{l \pi} r^{-p-n},
$$

with

$$
\begin{aligned}
& p \geqslant 1, \sum_{j=1}^{p} l_{j}=n \geqslant l \geqslant 0, \\
& \min \left|\sum_{j=1}^{p} \pm l_{j}\right| \leqslant l, \quad \pi=\pi_{1} \cdot \pi_{2} \cdots \pi_{p}, \\
& \mathfrak{T}^{l^{j_{j}}}=g_{A_{l_{j}}} \text { if } \pi_{j}=(-1)^{l_{j}}, \\
& \mathfrak{T}^{l_{j} \pi_{j}}=\epsilon_{i_{p q}} \mathrm{~S}_{p A_{l_{j-1}}} n_{q} \text { if } \pi=(-1)^{l_{j+1}}, \\
& {[\cdots]^{l \pi} \text { means "coupled to give a spherical harmonic }} \\
& \quad \text { of order } l \text { and parity } \pi \text { " (10.5e) }
\end{aligned}
$$

The general stationary metric coefficients $g_{\mu \nu}[\mathrm{Eq}$. (10.5)] are known to be analytic functions of the de Donder coordinates (Muller zum Hagen, 1970).

This general stationary metric in mass-centered de Donder gauge can be written more explicitly (but with some loss of information) as

$$
\begin{aligned}
& g_{00}=-1+\frac{2 g}{r}-\frac{2 g^{2}}{r^{2}} \\
&+\sum_{i=2}^{\infty} \frac{1}{r^{l+1}}\left[\frac{2(2 l-1) ! !}{l !} g_{A_{l}} N_{A_{l}}+[(l-1) \text { pole }]\right. \\
&+\cdots+[\text { monopole }]] \\
& g_{0 j}=\sum_{l=1}^{\infty} \frac{1}{r^{l+1}}\left\{-\frac{4 l(2 l-1) ! !}{(l+1) !} \epsilon_{j k a_{l}} s_{k A_{l-1}} N_{A_{l}}\right. \\
&+ {[(l-1) \text { pole }]+\cdots+[\text { monopole }]\} } \\
& g_{i j}= \delta_{i j}\left[1+\frac{2 g}{r}\right]+\frac{g^{2}}{r^{i}}\left(\delta_{i j}+n_{i} n_{j}\right) \\
&+ \sum_{l=2}^{\infty} \frac{1}{r^{l+1}}\left[\frac{2(2 l-1) ! !}{l !} g_{A_{l}} N_{A_{l}} \delta_{i j}+[(l-1) \text { pole }]\right.
\end{aligned}
$$

[The quadratic $g^{2} / r^{2}$ terms follow from Eq. (9.23) and from a similar equation for $\gamma_{j k}^{2000}$.]

Note that one can read the mass $l$-pole moment off the $1 / r^{l+1}$ part of $g_{00}$, and the current $l$-pole moment off the $1 / r^{l+1}$ part of $g_{0 j}$.

Janis and Newman (1965), Van der Burg (1968), Geroch (1970), Hansen (1974), and Clarke and Sciama (1971) have previously given definitions of multipole moments for stationary systems, and have proved that the moments completely fix the structure of the gravitational field, including its nonlinearities. However, it is not evident how the multipole moments which they define mesh with gravitational-wave theory, whereas the link of our moments to gravitational-wave theory is very simple and clear (Sec. XII). The moments in each set (Janis and Newman's, Van der Burg's, Geroch and Hansen's, Clarke and Sciama's, and mine) must be some combination of the moments in each other set. However, nobody has yet exhibited those relationships explicitly. 


\section{STATIONARY SOURCES IN AN "ACMC" COORDINATE SYSTEM}

\section{A. Motivation}

Suppose that one is given a stationary, asymptotically flat, vacuum metric in some arbitrary coordinate system. How can one compute its lowest few multipole moments?

One way would be to transform to a de Donder coordinate system, and then read off the moments by comparing with Eqs. (10.6). However, to perform such a coordinate transformation would be horrendously difficult for almost all metrics. (Try the Kerr metric, for example).

Fortunately, a full de Donder transformation is not necessary. There exists a wide class of coordinate systems in which one can readily read off the lowest few moments. I shall call such systems "asymptotically Cartesian and mass centered," or ACMC. It is rather straightforward to transform an arbitrary stationary metric into ACMC form and then read off its moments. The Kerr metric is treated as an example below (Sec. XI.D).

\section{B. Metric in ACMC coordinates}

A coordinate system will be called "asymptotically Cartesian and mass centered to order $N$ " (ACMC-N) if and only if the covariant metric components in it are time independent and have the following " $1 / r$ " and spherical harmonic structure:

$$
\begin{aligned}
g_{00}= & -1+\frac{2 g}{r}+\frac{(0 \text { pole })}{r^{2}} \\
+ & \sum_{l=2}^{N} \frac{1}{r^{l+1}}\left(\frac{2(2 l-1) ! !}{l !} g_{A_{l}} N_{A_{l}}\right. \\
& +(l-1 \text { pole })+\cdots+(0 \text { pole })) \\
& +\frac{1}{r^{N+2}}\left(\frac{2(2 N+1) ! !}{(N+1) !} g_{A_{N+1}} N_{A_{N+1}}\right. \\
& +(\text { poles with } l \neq N+1)) \\
& +\left(\text { terms that die out faster than } 1 / r^{N+2}\right) \\
g_{0 j}=\sum_{l=1}^{N} \frac{1}{r^{l+1}}[ & -\frac{4 l(2 l-1) ! !}{(l+1) !} \epsilon_{j k a_{l}} S_{k A} N_{A-1} \\
& +\left(l \text { pole with parity } \pi=(-1)^{l}\right) \\
& +(l-1 \text { pole })+\cdots+(0 \text { pole })]
\end{aligned}
$$

$$
\begin{aligned}
g_{00}=-1 & +\frac{2 M}{r}+\frac{(0 \text { pole })}{r^{2}}+\sum_{l=2}^{N} \frac{1}{r^{l+1}}\left[\frac{(2 l-1) ! !}{2}\left(\frac{2(l-1) l}{(l+1)(l+2)}\right)^{1 / 2} \sum_{m=-l}^{l} I^{l m} Y^{l m}+(l-1 \text { pole })+\cdots+(0 \text { pole })\right] \\
& +\frac{1}{r^{N+2}}\left[\frac{(2 N+1) ! !}{2}\left(\frac{2 N(N+1)}{(N+2)(N+3)}\right)^{1 / 2} \sum_{m=-(N+1)}^{(N+1)} I^{(N+1) m} Y^{(N+1) m}+(\text { poles with } l \neq N+1)\right] \\
& +\left(\text { terms that die out faster than } 1 / r^{N+2}\right)
\end{aligned}
$$

$$
\begin{aligned}
& g_{j k}=\delta_{j k}+\sum_{l=0}^{N} \frac{1}{r^{l+1}}[(l \text { pole })+(l-1 \text { pole }) \\
&+\cdots+(0 \text { pole })] \\
&+\frac{1}{r^{N+2}}\left[\left(\begin{array}{l}
\text { any angular } \\
\text { dependence }
\end{array}\right)\right]+\left(\begin{array}{l}
\text { terms that die out } \\
\text { faster than } r^{-(N+2)}
\end{array}\right) .
\end{aligned}
$$

Comparison of Eq. (11.1) with Eq. (10.6) shows that de Donder coordinates are ACMC $-N$ for all $N$-i.e., they are ACMC $-\infty$. Below (Sec. XI.C) we shall prove that for $l \leqslant N+1$ the STF coefficients $g_{A_{l}}$ and $S_{A_{l}}$ in expressions (11.1) are invariant under transformations from one ACMC- $N$ coordinate system to another. Since in de Donder coordinates $g_{A_{l}}$ and $S_{A_{l}}$ are the multipole moments of the source, this means that in any ACMC-N coordinate system the coefficients $g_{A_{l}}$ and $S_{A_{l}}$ with $l \leqslant N+1$ are the source's multipole moments.

One may prefer to use conventional spherical-harmonic notation rather than STF notation. The multipole moments $I^{l m}$ and $S^{l m}$ of conventional notation are related to those of STF notation by [cf. Eq. (4.7)]

$$
\begin{aligned}
& I^{l m}=\frac{16 \pi}{(2 l+1) ! !}\left(\frac{(l+1)(l+2)}{2(l-1) l}\right)^{1 / 2} \mathfrak{g}_{A_{l}} Y_{A_{l}}^{l m *}, \\
& S^{l m}=-\frac{32 \pi l}{(l+1)(2 l+1) ! !}\left(\frac{(l+1)(l+2)}{2(l-1) l}\right)^{1 / 2} S_{A_{l}} \mathcal{Y}_{A_{l} m *}^{l m *},
\end{aligned}
$$

where $\mathcal{Y}_{A_{l}}^{l m}$ are the STF tensors which generate the scalar spherical harmonics [Eqs. (2.12)] and * denotes complex conjugate. The inverse relation [Eqs. (4.6)] is

$$
\begin{aligned}
& g_{A_{l}}=\frac{l !}{4}\left(\frac{2(l-1) l}{(l+1)(l+2)}\right)^{1 / 2} \sum_{m=-l}^{l} I^{l m} \mathcal{Y}_{A_{l}}^{l m}, \\
& S_{A_{l}}=-\frac{(l+1) !}{8 l}\left(\frac{2(l-1) l}{(l+1)(l+2)}\right)^{1 / 2} \sum_{m=-l}^{l} S^{l m} \mathcal{Y}_{A_{l}}^{l m} .
\end{aligned}
$$

By comparing Eqs. (11.1), (11.3), (2.11), and (2.23b) $\mathrm{ACMC}-N$ coordinate system: 


$$
\begin{aligned}
g_{0 j}= & -\frac{2}{r^{2}} \epsilon_{j p q} S_{p} n_{q}+\frac{1}{r^{2}} \times\left(\begin{array}{c}
1 \text { pole with } \\
\text { parity } \pi=-1
\end{array}\right) \\
& +\sum_{l=2}^{N} \frac{1}{r^{l+1}}\left[\frac{-(2 l-1) ! !}{2}\left(\frac{2(l-1)}{l+2}\right)^{1 / 2} \sum_{m=-l}^{l} S^{l m} Y_{j}^{B, l m}+\left(\begin{array}{l}
l \text { pole with } \\
\text { parity } \pi=(-1)^{l}
\end{array}\right)+(l-1 \text { pole })+\cdots+(0 \text { pole })\right] \\
& +\frac{1}{r^{N+2}}\left[\frac{-(2 N+1) ! !}{2}\left(\frac{2 N}{N+3}\right)^{1 / 2} \sum_{m=-(N+1)}^{(N+1)} S^{(N+1) m} Y_{j}^{B,(N+1) m}+\left(\begin{array}{l}
N+1 \text { pole with } \\
\text { parity } \pi=(-1)^{N+1}
\end{array}\right)+(\text { poles with } l \neq N+1)\right] \\
& +\left(\text { terms that die out faster than } 1 / r^{N+2}\right), \\
g_{j k}= & \delta_{j k}+\sum_{l=0}^{N} \frac{1}{r^{l+1}}[(l \text { pole })+(l-1 \text { pole })+\cdots+(0 \text { pole })] \\
& +\frac{1}{r^{N+2}}\left[\left(\begin{array}{l}
\text { any angular } \\
\text { dependence }
\end{array}\right)\right]+\left(\text { terms that die out faster than } \frac{1}{r^{N+2}}\right) .
\end{aligned}
$$

Here $Y^{l m}=Y^{l m}(\theta, \phi)$ is the standard scalar spherical harmonic; $Y_{j}^{B, \imath \mathrm{m}}$ is the magnetic-parity pure-spin vector spherical harmonic of order $l$ [Eqs. (2.18b), (2.21b), $(2.22 \mathrm{~b})$, and (2.23b)]; $M=g$ is the mass of the source; and $S_{j}=\S_{j}$ is its intrinsic angular momentum.

\section{Transformations between ACMC coordinate systems}

We now study the coordinate transformations which link two ACMC-N coordinate systems, with the objective of proving the invariance of the multipole moments.

Let $\left\{x^{\alpha^{\prime}}\right\}$ and $\left\{x^{\alpha}\right\}$ both be ACMC $-N$ coordinate sys tems which are related by the coordinate transformation

$$
x^{\alpha^{\prime}}(\odot)=x^{\alpha}(\odot)+f^{\alpha}\left[x^{j}(\odot)\right],
$$

where $\rho$ is an event in spacetime. The $f^{\alpha}$ cannot depend on $x^{0}$ since we want $\partial / \partial x^{0}=\partial / \partial x^{0 \prime}=($ time-translation Killing vector $)$. We expand the functions $f_{\alpha}\left(x^{j}\right)$

$=\eta_{\alpha \beta} f^{\beta}\left(x^{j}\right)$ - and everything else in this section-in powers of $1 / \gamma$ and denote by $f_{\alpha}^{n}$ the part of order $r^{-(n+1)}$,

$$
\begin{aligned}
& f_{0}=\sum_{n=-1}^{\infty} r^{-(n+1)} f_{0}^{n}(\theta, \phi), \\
& f_{j}=\sum_{n=-1}^{\infty} r^{-(n+1)} f_{j}^{n}(\theta, \phi) .
\end{aligned}
$$

The expansion cannot include $n<-1$; if it did, then one or both coordinate systems would violate $g_{\alpha \beta}-\eta_{\alpha \beta}$ $+O(1 / r)$ as $r \rightarrow \infty$. We then expand the angular functions $f_{0}^{n}$ in scalar spherical harmonics $Y^{l m}$ denoting by $l_{n}$ the maximum order that appears in the expansion; and we expand the $f_{j}^{n}$ in pure-orbital vector harmonics $Y_{j}^{l^{\prime \prime}, l m}$ $\equiv Y_{j}^{(2+J), l m}$ (Sec. II. D), denoting by $l_{n J}$ the maximum order that appears:

$$
f_{0}^{n}=\sum_{i=0}^{l} f_{0}^{n l}(\theta, \phi), f_{j}^{n}=\sum_{J=-1}^{1} \sum_{i=0}^{l_{n} J} f_{j}^{n J}(\theta, \phi) .
$$

Here $f_{0}^{n l}$ is a linear combination (sum over $m$ with suitable coefficients) of $Y^{l m}$; and $f_{j}^{n J l}$ is a linear combination of $Y_{j}^{l+J, l m}$. We seek to determine the values of $l_{n}$ and $l_{n J}$ which make $f_{\alpha}$ the most general coordinate transformation linking two ACMC $-N$ coordinate systems.

We define the functions $h_{\alpha \beta}$ by

$$
h_{\alpha \beta}\left(x^{j^{\prime}}\right) \equiv g_{\alpha \cdot \beta^{\prime}}\left(x^{j^{\prime}}\right)-\eta_{\alpha \beta}
$$

and we expand $h_{\alpha \beta}\left(x^{j}\right)$-the same functions but with $x^{j}$ replaced by $x^{j}$-in powers of $1 / r$,

$$
h_{\alpha \beta}\left(x^{j}\right)=\sum_{n=0}^{\infty} r^{-(n+1)} h_{\alpha \beta}^{n}(\theta, \phi) \text {. }
$$

The ACMC $-N$ nature of the $\left\{x^{\alpha^{\prime}}\right\}$ coordinates is equivalent to (i) the demand that no terms with $n<0$ appear in Eq. (11.7b), plus (ii) the following spherical-harmonic properties of the $h_{\alpha \beta}^{n}$ :

$h_{00}^{0}$ and $h_{j k}^{0}$ contain only monopoles; $h_{0 j}^{0} \equiv 0$;

$h_{0 j}^{1}$ and $h_{j k}^{1}$ contain monopoles and dipoles; $h_{00}^{1}$ contains only monopoles;

$h_{\alpha \beta}^{n}$ contains only poles of order $l \leqslant n$

$$
\text { for } 2 \leqslant n \leqslant N \text {. }
$$

We expand $h_{\alpha \beta}^{n}$ in spherical harmonics subject to these constraints

$$
h_{\alpha \beta}^{n}=\sum_{K, l} h_{\alpha \beta}^{n K l}(\theta, \phi) .
$$

Here $K$ denotes the type of pure-orbital harmonic (scalar, or vector with given value of $l^{\prime}$, or tensor with given values of $\lambda$ and $l^{\prime}$, see Sec. II); and $l$ denotes the order of the harmonic.

The coordinate transformation (11.5) produces the following relationship between $g_{\mu \nu}(x)$ and $g_{\alpha^{\prime} \beta^{\prime}}\left(x^{\prime}\right)$ :

$$
g_{\mu \nu}(x)=\frac{\partial x^{\alpha^{\prime}}}{\partial x^{\mu}} \frac{\partial x^{\beta^{\prime}}}{\partial x^{\nu}} g_{\alpha^{\prime} \beta \cdot}\left[x^{\prime}(x)\right] \text {. }
$$

Upon inserting expression (11.7a) for $g_{\alpha^{\prime} \beta^{\prime}}$ and expression (11.5) for $x^{\prime}(x)$, we obtain

$$
\begin{aligned}
& g_{\mu \nu}(x)=\eta_{\mu \nu}+h_{\mu \nu}(x)+a_{\mu \nu}(x)+b_{\mu \nu}(x), \\
& a_{\mu \nu}(x) \equiv\left(\delta^{\alpha}{ }_{\mu}+f^{\alpha}{ }_{, \mu}\right)\left(\delta^{\beta}{ }_{\nu}+f^{\beta}{ }_{, \nu}\right) \\
& \times \sum_{n=0}^{\infty}(n !)^{-1} f_{j_{1}} f_{j_{2}} \cdots f_{j_{n}} \nabla_{j_{1}} \nabla_{j_{2}} \cdots \nabla_{j_{n}} h_{\alpha \beta}(x) \\
& -h_{\mu \nu}(x)+f^{\alpha}{ }_{, \mu} f_{\alpha, \nu}, \\
& \boldsymbol{b}_{\mu \nu}(x) \equiv f_{\mu, \nu}+f_{\nu, \mu} \cdot
\end{aligned}
$$

Here $\nabla_{j}$ is the covariant derivative with respect to the flat background metric $\nabla_{j} h_{\alpha \beta} \equiv h_{\alpha \beta, j}$; cf. Eq. (1.13) and associated discussion. We now expand $g_{\mu \nu}, a_{\mu \nu}$ and $b_{\mu \nu}$ in powers of $(1 / r)$ in the manner of Eq. (11.6a) 


$$
\begin{aligned}
g_{\mu \nu} & =\eta_{\mu \nu}+\sum_{n=0}^{\infty} r^{-(n+1)} g_{\mu \nu}^{n}(\theta, \phi), \\
a_{\mu \nu} & =\sum_{n=0}^{\infty} r^{-(n+1)} a_{\mu \nu}^{n}(\theta, \phi), \\
b_{\mu \nu} & =\sum_{n=0}^{\infty} r^{-(n+1)} b_{\mu \nu}^{n}(\theta, \phi) .
\end{aligned}
$$

Then the demand that $\left\{x^{\mu}\right\}$, like $\left\{x^{\alpha^{\prime}}\right\}$ be $\mathrm{ACMC}-N$ is equivalent to the demand that $g_{\mu \nu}^{n}$ satisfy the same conditions (11.8) as $h_{\mu \nu}^{n}$-and this, by virtue of Eq. (11.11a), is equivalent to the demand that

$a_{00}^{0}+b_{00}^{0}$ and $a_{j k}^{0}+b_{j k}^{0}$ contain only monopoles;

$$
a_{0 j}^{0}+b_{0 j}^{0}=0 \text {; }
$$

$a_{0 j}^{1}+b_{0 j}^{1}$ and $a_{j k}^{1}+b_{j k}^{1}$ contain only monopoles and dipoles ; $a_{00}^{1}+b_{00}^{1}$ contain only monopoles ;

$a_{\alpha \beta}^{n}+b_{\alpha \beta}^{n}$ contain only harmonics with $0 \leqslant l \leqslant n$

$$
\text { for } 2 \leqslant n \leqslant N \text {. }
$$

We shall now determine the limiting $l$ values, $l_{n}$ and $l_{n J}$, which are equivalent to these ACMC $-N$ conditions, and in the process we shall prove the equality of the multipole moments which one reads off the two ACMC$N$ coordinate systems.

We begin with conditions (11.13a) on $a_{\mu \nu}^{0}+b_{\mu \nu}^{0}$. Equations (11.11b), (11.7b), and (11.6a) imply that $a_{\mu \nu} \sim 1 / r^{2}$, so $a_{\mu \nu}^{0}=0$. Equations $(11.11 \mathrm{c})$ and $(11.6)$ imply

$$
\begin{aligned}
& b_{00}^{0}=0, \quad b_{0 j}^{0}=\sum_{l=0}^{l-1} r f_{0, j}^{-1 l}, \\
& b_{j k}^{0}=\sum_{j=-1}^{1} \sum_{l=0}^{l(-1) J} 2 v f_{(j, k)}^{-1 J l} .
\end{aligned}
$$

Since an $l=0$ scalar harmonic is constant but all others depend on $\theta$ and $\phi$, the condition $a_{0 j}^{0}+b_{0 j}^{0}=b_{0 j}^{0}=0$ is equivalent to

$$
l_{-1}=0 \text {. }
$$

For $b_{j k}^{0}$ the situation is more delicate. For fixed $l \geqslant 2$ the three different types of vector harmonics $Y_{j}^{l+J, l m}$ $(J=0, \pm 1)$ have linearly independent gradients $Y_{(j, k)}^{l+J j m}$; but for $l=1 Y_{(j, k)}^{0,1 m}$ vanishes, while $Y_{(j, k)}^{1,1 m}$ and $Y_{(j, k)}^{2,1 m}$ are linearly independent [see Eq. (2.24)]. This fact, together with Eqs. (11.14) and $a_{j k}^{0}=0$, means that $\left\{a_{j k}^{0}+b_{j k}^{0}\right.$ contains only monopoles $\}$ is equivalent to

$$
l_{(-1)(-1)}=1 ; \quad l_{(-1) 0}=l_{(-1) 1}=0 .
$$

Thus, conditions (11.15) are equivalent to the $n=0$ ACMC $-N$ conditions (11.13a). Moreover, when these conditions are imposed (and even if they are not!) $a_{00}^{0}+b_{00}^{0}$ vanishes - which means that both coordinate systems exhibit the same mass monopole moment.

We turn next to conditions (11.13b) on $a_{\mu \nu}^{1}+b_{\mu \nu}^{1}$. Equations (11.11), (11.6), (11.7b), (11.15b), and the fact that $h_{00}^{0}$ is a constant (the mass monopole), imply

$$
\begin{aligned}
a_{00}^{1}+b_{00}^{1} & =a_{00}^{1}=r^{2}\left(f_{j}^{-1} \nabla_{j}\right)\left(r^{-1} h_{00}^{0}\right) \\
& =-\left(\sum_{J} f_{j}^{-1 J 0} n_{j}+f_{j}^{(-1)(-1) 1} n_{j}\right) h_{00}^{0} .
\end{aligned}
$$

The first term has $l=0$ and is thus acceptable; but the second has $l=1$ in violation of conditions (11.13b). To preserve $(11.13 \mathrm{~b})$ we must set $f_{j}^{(-1)(-1) 1}=0-$ i.e., we must revise downward our value of $l_{(-1)(-1)}$ :

$$
l_{(-1)(-1)}=0 \text {. }
$$

Returning to Eqs. (11.11), (11.6), (11.15a), (11.7b), and (11.8) we infer that

$$
a_{0 j}^{1}+b_{0 j}^{1}=b_{0 j}^{1}=r^{2} \nabla_{j}\left(r^{-1} \sum_{i=0}^{l_{0}} f_{0}^{0 l}\right) .
$$

This will be pure monopole and pure dipole [condition $(11.13 \mathrm{~b})]$ if and only if

$$
l_{0}=1 \text {. }
$$

Returning again to Eqs. (11.11), and combining with Eqs. (11.7b), (11.6a), and (11.15a) we see

$$
\begin{aligned}
a_{j k}^{1}+b_{j k}^{1}=r^{2} & {\left[\left(f_{i}^{-1} \nabla_{i}\right)\left(r^{-1} h_{j k}^{0}\right)+\left(f_{i, j}^{-1}\right)\left(r^{-1} h_{i k}^{0}\right)\right.} \\
& +\left(f_{i, k}^{-1}\right)\left(r^{-1} h_{i j}^{0}\right)+\left(f_{i, j}^{-1}\right)\left(f_{i, k}^{-1}\right) \\
& \left.+\left(r^{-1} f_{j}^{0}\right)_{, k}+\left(r^{-1} f_{k}^{0}\right)_{, j}\right] .
\end{aligned}
$$

Since $f_{i}^{-1}$ and $h_{i j}^{0}$ are both pure monopole, the first four terms are pure monopole and thus acceptable. The last two terms, by virtue of Eqs. $(11.6 \mathrm{~b})$, reduce to

$$
\sum_{j=-1}^{1} \sum_{i=0}^{l_{0 J} J}\left[\left(r^{-1} f_{j}^{0 J \imath}\right)_{, k}+\left(r^{-1} f_{k}^{0 J}\right)_{, j}\right]
$$

which is monopole and dipole (the required form) if and only if

$$
l_{0 J}=1 \text {. }
$$

Equations (11.17a)-(11.17c) are thus equivalent to the $n=1$ ACMC $-N$ conditions (11.13b). Moreover, $a_{0 j}^{1}+b_{0 j}^{1}$ as given by Eqs. (11.18) involves only harmonics of electric-type parity $\pi=(-1)^{l}$. Thus, the two coordinate systems exhibit the same magnetic-type $l=1$ component of $g_{0 j}^{1}$-i.e., the same current dipole moment (angular momentum). And since both systems are mass centered ( $g_{00}^{1}$ pure monopole), both exhibit vanishing mass dipole moments.

Turn next to conditions (11.13c) on $a_{\alpha \beta}^{n}+b_{\alpha \beta}^{n}$ for $n \leqslant N$. We shall use induction to prove the following:

(i) the indices $l_{n-1}$ and $l_{(n-1) J}$ satisfy

$$
l_{n-1}=l_{(n-1) J}=n \text {, }
$$

and (ii) the two coordinate systems exhibit the same mass and current moments of order $n$. We have already proved these properties for $n=1$. We now as sume that they have been proved for $1,2, \ldots(n-1)$, and we then prove them for order $n$ : Equations (11.11b), together with the fact that gradients do not change multipole order, together with the expansions (11.6), (11.7), (11.9), and (11.12) imply that $a_{\mu \nu}^{n}$ contains the following types of terms:

$$
\begin{aligned}
& f_{j_{1}}^{p_{1} J_{1} l_{1}} f_{j_{2}}^{p_{2} J_{2} l_{2}} \text {, with } p_{1}+p_{2}+3=n,
\end{aligned}
$$

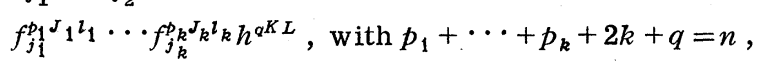

$$
\begin{aligned}
& k \geqslant 1 \text {. (11.20b) }
\end{aligned}
$$

Here wherever $f_{j_{i}}^{p_{i} J_{i}} l_{i}$ is written, the term could equally well be $f_{0}^{p_{i} l_{i}}$ (i.e., scalar harmonic rather than vector). The first term, when coupled to form pure harmonics, 
gives harmonics of order $l \leqslant l_{1}+l_{2}$; and since Eq. (11.20a) together with $p_{i} \geqslant-1$ says $p_{1} \leqslant n-2, p_{2} \leqslant n-2$ we can use our induction assumption to infer that

$$
l \leqslant l_{1}+l_{2} \leqslant l_{p_{1} J_{1}}+l_{p_{2} J_{2}}=p_{1}+1+p_{2}+1=n-1 .
$$

Thus, the first term contains only terms of order $l \leqslant n-1$-which is permissible under the ACMC $-N$ constraints (11.13c). Similarly Eqs. (11.20b), together with the induction hypothesis and the ACMC $-N$ property $L \leqslant q$ of $h_{\alpha \beta}^{q K L}$, implies that the second term [Eq.(11.20b)] consists of harmonics

$$
\begin{aligned}
l & \leqslant l_{1}+\cdots+l_{k}+L \leqslant l_{p_{1} J_{1}}+\cdots+l_{p_{k^{J}}}+L \\
& \leqslant p_{1}+\cdots+p_{k}+k+q=n-k \leqslant n-1 .
\end{aligned}
$$

Thus, the second term contains only harmonics of order $l \leqslant n-1$-which is permissible under the ACMC $-N$ constraints $(11.13 \mathrm{c})$. Since these two terms in $a_{\mu \nu}^{n}$ present no problems, we turn to $b_{\mu \nu}^{n}$. Equations (11.11c) and (11.6) imply

$$
\begin{aligned}
& b_{00}^{n}=0, \\
& b_{0 j}^{n}=\sum_{l=0}^{l_{n-1}} r^{n+1}\left[r^{-n} f_{0}^{(n-1) l}\right]_{, j}, \\
& \left.b_{j k}^{n}=\sum_{J} \sum_{i=0}^{l(n-1) J} 2 r^{n+1}\left[r^{-n} f_{(j}^{(n-1) J l}\right]_{k}\right) .
\end{aligned}
$$

By virtue of these relations the $\mathrm{ACMC}-N$ conditions (11.13c) are equivalent to conditions (11.19) on the indices $l_{(n-1) J}$ and $l_{(n-1)}$; and Eqs. (11.21a) and (11.21b), together with the fact that $a_{\alpha \beta}^{n}$ contains only harmonics of order $l \leqslant n-1$, implies that the two coordinate systems will exhibit the same mass $n$-pole moment $(l=n$ part of $h_{00}^{n}$ ) and the same current $n$-pole moment $\left(\pi=(-1)^{l+1}, l=n\right.$ part of $\left.h_{0 j}^{n}\right)$. This completes our inductive proof of properties (i) and (ii) above, for $n \leqslant N$.

If we turn our attention to $n=N+1$, the constraints (11.13c) no longer hold, so $l_{n}$ and $l_{n J}$ can be arbitrarily large. Nevertheless, Eqs. (11.21) still hold, and $a_{\alpha \beta}^{n}$ still contains harmonics only of order $l \leqslant n-1$. Consequently it is still true for $n=N+1$ (but not for $n=N+2$ ) that the mass and current $n$-pole moments exhibited by the two coordinate systems are the same.

\section{Multipole moments of the Kerr metric}

I shall now illustrate, using the Kerr metric, how one us es ACMC coordinate systems to compute the multipole moments of a stationary metric.

In the "flat-space normalized" basis

$$
\begin{aligned}
& \mathbf{e}_{0}=\partial_{t}, \quad \mathbf{e}_{r}=\partial_{r}, \quad \mathbf{e}_{\theta}=r^{-1} \partial_{\theta}, \\
& \mathbf{e}_{\phi}=(r \sin \theta)^{-1} \partial_{\phi},
\end{aligned}
$$

associated with Boyer-Lindquist coordinates, the nonzero metric coefficients of the Kerr metric are (cf. p. 877 of MTW

$g_{00}=\frac{-r^{2}+2 m r-a^{2} \cos ^{2} \theta}{r^{2}+a^{2} \cos ^{2} \theta}=-1+\frac{2 m}{r}-\frac{2 m a^{2} \cos ^{2} \theta}{r^{3}}+O\left(\frac{1}{r^{5}}\right)$,

$$
\begin{aligned}
g_{0 \phi}= & -\frac{2 m a \sin \theta}{r^{2}+a^{2} \cos ^{2} \theta}=-\frac{2 m a \sin \theta}{r^{2}} \\
& +\frac{2 m a^{3} \sin \theta \cos ^{2} \theta}{r^{4}}+O\left(\frac{1}{r^{6}}\right), \\
g_{r r}= & \frac{r^{2}+a^{2} \cos ^{2} \theta}{r^{2}-2 m r+a^{2}}=1+\frac{2 m}{r}+\frac{4 m^{2}-a^{2} \sin ^{2} \theta}{r^{2}} \\
& +\frac{8 m^{3}-2 m a^{2}\left(2-\cos ^{2} \theta\right)}{r^{3}}+O\left(\frac{1}{r^{4}}\right), \\
g_{\theta \theta}= & \frac{r^{2}+a^{2} \cos ^{2} \theta}{r^{2}}=1+\frac{a^{2}-a^{2} \sin ^{2} \theta}{r^{2}}, \\
g_{\phi \Phi}= & \frac{r^{2}+a^{2}}{r^{2}}+\frac{2 m}{r}\left(\frac{a^{2} \sin ^{2} \theta}{r^{2}+a^{2} \cos ^{2} \theta}\right) \\
= & 1+\frac{a^{2}}{r^{2}}+\frac{2 m a^{2} \sin ^{2} \theta}{r^{3}}+O\left(\frac{1}{r^{5}}\right) .
\end{aligned}
$$

(For discussion of how one uses spherical coordinates, such as these, interchangeably with Cartesian coordinates, see Sec. I.C.)

Since $g_{00}$ has no $1 / r^{2}$ terms, the coordinates are mass centered. At order $(1 / r) g_{\alpha \beta}$ contains only monopoles; but at $O\left(1 / r^{2}\right)$ it contains monopoles, dipoles and quadrupoles (the $-a^{2} \sin ^{2} \theta / r^{2}$ terms in $g_{r r}$ and $g_{\theta \theta}$ are both quadrupole). Thus, the coordinates are only ACMC-0, which means that we can read off only the mass monopole moment and the current dipole moment of the source. Direct comparison of Eqs. (11.23a) and (11.23b) with Eqs. (11.1a) and (11.1b) or (11.4a) and (11.4b) reveals that these moments are

$$
\begin{aligned}
& M \equiv \mathfrak{g}=m=\text { mass } ; \\
& \mathbf{S} \equiv \mathbf{S}=m a \mathbf{e}_{\mathbf{z}}=\text { (angular momentum) } .
\end{aligned}
$$

To determine the quadrupole moments we must transform to an ACMC-1 coordinate system-i.e., we must get rid of the offending $-a^{2} \sin ^{2} \theta / r^{2}$ quadrupole terms in $g_{r r}$ and $g_{\theta \theta}$. This can be accomplished by the transformation

$$
\begin{aligned}
& r=r^{\prime}+a^{2} \cos ^{2} \theta^{\prime} / 2 r^{\prime}, \theta=\theta^{\prime}-a^{2} \cos \theta^{\prime} \sin \theta^{\prime} / 2 r^{\prime 2}, \\
& \phi=\phi^{\prime}, \quad t=t^{\prime},
\end{aligned}
$$

which leads to

$$
\begin{aligned}
g_{0^{\prime} 0^{\prime}} & =-1+\frac{2 m}{r^{\prime}}-\frac{3 m a^{2} \cos ^{2} \theta^{\prime}}{r^{\prime 3}}+O\left(\frac{1}{r^{\prime 5}}\right) \\
& =-1+\frac{2 m}{r^{\prime}}-\frac{2 m a^{2}}{r^{\prime 3}}\left[\frac{1}{2}+P^{2}\left(\cos \theta^{\prime}\right)\right]+O\left(\frac{1}{r^{\prime 5}}\right),
\end{aligned}
$$

$$
\begin{aligned}
g_{0^{\prime} \phi^{\prime}}= & -\frac{2 m a \sin \theta^{\prime}}{r^{\prime 2}}+\frac{5 m a^{3} \sin \theta^{\prime} \cos ^{2} \theta^{\prime}}{r^{\prime 4}}+O\left(\frac{1}{r^{\prime 6}}\right) \\
= & \frac{2 m a P^{1} \theta_{\theta^{\prime}}}{r^{\prime 2}}-\frac{m a^{3}}{r^{\prime 4}}\left(P_{, \theta^{\prime}}^{1}+\frac{2}{3} P^{3}, \theta^{\prime}\right)+O\left(\frac{1}{r^{\prime 6}}\right), \\
g_{r^{\prime} r^{\prime}}= & 1+\frac{2 m}{r^{\prime}}+\frac{4 m^{2}-a^{2}}{r^{\prime 2}}+\frac{8 m^{3}-4 m a^{2}-m a^{2} \cos ^{2} \theta^{\prime}}{r^{\prime 3}} \\
& +O\left(\frac{1}{r^{\prime 4}}\right), \\
g_{\theta^{\prime} \theta^{\prime}}= & 1+\frac{a^{2}}{r^{\prime 2}}+O\left(\frac{1}{r^{\prime 4}}\right),
\end{aligned}
$$




$$
\begin{aligned}
& g_{\phi^{\prime} \phi^{\prime}}=1+\frac{a^{2}}{r^{\prime 2}}+\frac{2 m a^{2} \sin ^{2} \theta^{\prime}}{r^{\prime 3}}+O\left(\frac{1}{r^{\prime 4}}\right), \\
& g_{r^{\prime} \theta^{\prime}}=-\frac{2 m a^{2} \cos \theta^{\prime} \sin \theta^{\prime}}{r^{\prime 3}}+O\left(\frac{1}{r^{\prime 4}}\right) .
\end{aligned}
$$

Here $P^{l}=P^{l}(\cos \theta)$ is the Legendre polynomial of order $l$. Not only are we now rid of the $1 / r^{2}$ quadrupole terms; we also have a $1 / r^{3}$ part devoid of octupole and higherorder terms. Thus, this coordinate system is ACMC-2; and we can read off the mass and current quadrupole and octupole moments. Comparison of expressions (11.26) and (11.4), and invocation of the spherical-harmonic relations $(2.7),(2.8),(2.18 \mathrm{~b})$, and (2.19), reveals

$$
\begin{aligned}
& I^{20}=-4\left(\frac{4 \pi}{15}\right)^{1 / 2} m a^{2}, I^{2 \mu}=0 \text { if } \mu \neq 0, S^{2 \mu}=0 ; \\
& S^{30}=\frac{4}{3}\left(\frac{4 \pi}{105}\right)^{1 / 2} m a^{3}, S^{3 \mu}=0 \text { if } \mu \neq 0, I^{3 \mu}=0 .
\end{aligned}
$$

These same moments in STF notation, as computed from Eqs. (11.3) and (2.12), are

$$
\begin{aligned}
g_{x x}= & g_{y y}=\frac{1}{3} m a^{2}, \quad g_{z z}=-\frac{2}{3} m a^{2}, \\
& \quad \text { all other } g_{j k}=0 ; \\
S_{j k}= & 0, \quad g_{j k l}=0 ; \\
S_{z z z}= & -\frac{4}{15} m a^{3}, \\
S_{z x x}= & S_{x z x}=S_{x x z}=S_{z y y}=S_{y z y}=S_{y y z}=\frac{2}{15} m a^{3} .
\end{aligned}
$$

Note that these moments have the following properties: $g_{A_{l}}=0$ if $l$ is odd; $g_{A_{l}}=$ const. $\times m a^{l}$ if $l$ is even; $\boldsymbol{S}_{A_{l}}=0$ if $l$ is even; $\delta_{A_{l}}=$ const. $\times m a^{l}$ if $l$ is odd. Janis and New man (1965) and Hansen (1974) find these same properties for their versions of the multipole moments - as does Hernandez (1967), who defines moments only for the Kerr metric and not for general spacetimes. This suggests that there might be some simple general relationship between our moments and theirs.

\section{FORMALISM FOR CALCULATING WAVE GENERATION}

\section{A. The formalism}

The foundations are now laid. Redemption is at hand.

Consider any system with slow internal motions

$$
\begin{aligned}
& R \lessgtr L \equiv(\text { size of source }) \\
& \ll x=\left(\begin{array}{l}
\text { time scale for change of } \\
\text { external gravitational field }
\end{array}\right), \\
& M \ll x .
\end{aligned}
$$

One can calculate the gravitational waves the system emits by the following procedure: (i) Analyze the structure and evolution of the system in any convenient coordinate system and by any fairly accurate approximation scheme. (ii) From that analysis obtain an approximation to the external gravitational field which, at any moment, satisfies (to some degree of accuracy) the timeindependent, vacuum Einstein field equations. (iii) By transforming that external field to an ACMC coordinate system, read off its dominant multipole moments (the moments with the largest values of $\left.{ }^{(l)} \mathfrak{M}^{l \pi}\right)$. (iv) Plug those dominant moments into the gravitational-wave formulas of Sec. IV.

That this calculational procedure will give a good approximation to the waves actually emitted, one can see as follows: Suppose that one were to compute the structure and evolution of the system in de Donder gauge. Then the radiation field would be described, to fractional accuracy $(M / \chi) \ln (\chi / R)$, by the formulas of Sec.IV with the near-zone, de Donder multipole moments fed in (for proof see Sec. IX.H). The near-zone, de Donder moments are determined uniquely by the "stationary part" $g_{\mu \nu}^{\bullet 0 \cdot}$ of the source's metric [cf. Eqs. (9.16) and (9.17)] -which stationary part approximates the full metric in and near the source, to within fractional errors $L / \chi$ and $M / \chi$. In the source's exterior $g_{\mu \nu}^{\bullet{ }^{0}}$ satisfies the time-independent vacuum field equations; so its multipole moments can be read off its line element in any ACMC coordinate system (for proof see Sec.XI). If the approximation scheme chosen for steps (i) and (ii) of the calculational procedure gives an external metric that closely approximates $g_{\mu \nu}^{\bullet \cdot}$, then the moments computed in step (iii) will closely approximate those of $g_{\mu \nu}^{\bullet 0 \cdot}$-i.e., they will closely approximate the de Donder moments; and, consequently step (iv) of the procedure will give a good approximation to the radiation.

One attractive approximation scheme for use in steps (i) and (ii) is the "instantaneous-gravity" approximation. In this scheme one sets to zero all time derivatives of the metric (but not of the matter variables) when solving the Einstein field equations $G_{\mu \nu}=8 \pi T_{\mu \nu}$. This has the effect of removing all dynamical freedom from the gravitational field and making gravitational interactions within the source instantaneous rather than retarded. One automatically obtains an external gravitational field which satisfies the time-independent vacuum field equations; and, unless one has made a foolish choice of coordinate system, the moments which one computes from that external field should contain errors no larger than

$$
\frac{L}{x} \sim\left(\begin{array}{l}
\text { error due to ignoring } \\
\text { retardation across source }
\end{array}\right) \text {. }
$$

The radiation field computed from those moments will then have fractional errors $L / \lambda$ and $(M / \chi) \ln (\chi / R)$.

\section{B. Examples of applications}

The shear modulus of the crust of a neutron star presumably is small enough that the eigenperiods of torsional oscillations of the crust are long compared with the light-travel time across the star. Hence, for such torsional oscillations the instantaneous -gravity approximation and the above slow-motion wave-generation formalism should be fairly accurate. Schumaker and Thorne (1980) analyze torsional oscillations using these formalisms.

These formalisms are also nicely suited to slowly rotating, deformed neutron stars (e.g., the Crab pulsar). The analysis of such stars using these formalisms would closely parallel Ipser's (1970) analysis and, of course, would produce the same results.

Consider two black holes in a binary orbit around each other, with the distance of closest approach large 
compared to their Schwarzschild radii. The analysis by D'Eath (1975), which used matched asymptotic expansions, shows that the orbital motion will have the same Keplerian form as for two normal stars with the same masses as the holes. Application of the above formalism then shows that the gravitational waves emitted will be the same as for a binary system made of normal stars [see Peters and Mathews (1963)].

\section{ACKNOWLEDGMENT}

For helpful discussions during the early stages of this work I thank James R. Ipser.

\section{APPENDIX: TAILS OF THE GRAVITATIONAL WAVES}

In the paragraph preceding Eq. (9.24) we discussed the matching of the near-zone gravitational field onto outgoing waves. That discussion sloughed over delicacies associated with "tails" of the outgoing waves. We describe those delicacies here.

The near-zone tails are caused by nonlinearities in the wave zone. If one ignores those nonlinearities-i.e., if one sets $W_{\alpha \beta}$ (Eq. 9.3) to zero in the wave zone-then the wave-zone field has the linear form (9.31), and matching of wave zone to near zone then generates "nontail" near-zone terms of the form discussed in the text (Eqs. 9.24, 9.25, 9.28). If, on the other hand, one computes the tiny nonlinear corrections $\gamma_{\alpha \beta}^{2}, \gamma_{\alpha \beta}^{3}, \ldots$ to the wave-zone field using the procedure of Eqs. (9.7), one discovers that they include terms in which the outgoing $\gamma_{\alpha \beta}^{1}$ scatters off itself to produce ingoing waves. These ingoing waves get converted in the near zone back into outgoing waves, and the resulting "matching-generated" near-zone fields contain terms such as

$$
\gamma_{\mu \nu}^{\text {tail }}=\text { const. } \times \int_{-\infty}^{t} \frac{g \mathfrak{g}\left\{\pi\left(t^{\prime}\right) r^{\ell^{\prime}}\right.}{\left(r+t-t^{\prime}\right)^{\boldsymbol{r}^{\prime+t^{\prime}+3}}} d t^{\prime} .
$$

These are the tails of the outgoing waves; they are characterized by a power-law fall-off in the time $t-t^{\prime}$ since generation of the waves. In the specific tail term (A1) the contribution from time $t^{\prime}$ results from the outgoing wave $\gamma_{\mu \nu}^{1} \sim\left(1 / \gamma^{\prime \prime}\right)^{(\ell)} \mathfrak{T N}^{k \pi}\left(t^{\prime \prime}-r^{\prime \prime}\right)$ backscattering, at retarded time $t^{\prime \prime}-r^{\prime \prime}=t^{\prime}$ and at radius $r^{\prime \prime}=\left(r+t-t^{\prime}\right) / 2$, off the static monopole field $\gamma_{\mu \nu}^{1} \sim g / \gamma^{\prime \prime}$. For details of how this backscatter leads, through matching, to a near-zone tail of the form (A1) see, e.g., Price (1972), Thorne (1972), Bardeen and Press (1973).

In the "transition region" $r \sim \lambda$ (Eq. 9.30), where our matching of wave zone to near zone occurs, and at epochs when the radiation is strongest, the tail termsbeing nonlinear-are small compared to the linear-order outgoing waves. More specifically, for the dominant multipoles of the radiation field-the ones with the largest values of $\left.\right|^{(l)} \mathfrak{T}^{l \pi} \mid$ - the largest tail terms are of the form (A1) and have magnitude

$$
\left|\gamma_{j k}^{\operatorname{tai1}}\right| \leq\left.\right|^{(l)} \mathfrak{M N}^{l \pi} / \lambda|(M / \lambda) \sim|\left(\gamma_{j k}^{1}\right)^{T T} \mid(M / \lambda)
$$

at $r \sim \pi$ and at times $t$ when the radiation is strongest. In the discussion of Eqs. (9.32)-(9.35) these tail terms will lead to fractional errors

$$
\left|\sin ^{2 \pi}\right| /\left|\mathfrak{T}^{2 \pi}\right| \lesssim M / \pi
$$

in the computation of the dominant multipole moments, and to fractional errors

$$
\left|\delta \bar{h}_{j k}^{T T}\right| /\left|\left(\gamma_{j k}^{1}\right)^{T T}\right| \leq M / \lambda
$$

in the computation of the gravitational-wave field. Thus, the tails do not change Eqs. (9.34) and (9.35).

\section{REFERENCES}

Ashtekar, A., and R. O. Hansen, 1978, J. Math. Phys. 19, 1542.

Bardeen, J. M. , and W. H. Press, 1973, J. Math Phys. 14, 7. Bondi, H. , M. G. J. van der Burg, and A. W. K. Metzner, 1962, Proc. R. Soc. London Ser. A 269, 21.

Bonnor, W. B., and M. A. Rotenberg, 1966, Proc. R. Soc. London Ser. A 289, 247.

Bontz, R. J., and R. H. Price, 1979, Astrophys. J. 228, 560. Burke, W. L., 1969, unpublished Ph. D. thesis, California Institute of Technology.

Burke, W. L., 1971, J. Math. Phys. 12, 401.

Campbell, W. B., and T. Morgan, 1971, Physica (Utrecht) $53,264$.

Campbell, W. B., J. Macek, and T. A. Morgan, 1977, Phys. Rev. D 15, 2156.

Chandrasekhar, S., and F. P. Esposito, 1970, Astrophys. J. 160, 153.

Chandrasekhar, S., and Y. Nutku, 1969, Astrophys. J. 158, 55.

Chrzanowski, P. L., and C. W. Misner, 1974, Phys. Rev. D 10, 1701.

Clarke, C. J. S., and D. W. Sciama, 1971, Gen. Relativ. Gravit. 2, 331.

Cooperstock, F. I., and D. J. Booth, 1969, Nuovo Cimento B 62, 163.

Cosgrove, C., 1980, Phys. Rev. D, in press.

Courant, R., and D. Hilbert, 1953, Methods of Mathematical

Physics, Vol. 1 (Interscience, New York), pp. $510 \mathrm{ff}$.

Curtis, G. E., 1978, Proc. R. Soc. London Ser. A 359, 133. D'Eath, P. D., 1975, Phys. Rev. D 12, 2183.

DeWitt, B. S., 1971, Lectures on Relativity-Stanford, Fall 1971, unpublished manuscript.

Dixon, W. G., 1970, Proc. R. Soc. London Ser. A 319, 509.

Dixon, W. G., 1977, in Isolated Gravitating Systems in Geneval Relativity, edited by J. Ehlers (North-Holland, Amsterdam).

Eardley, D. M. , D. L. Lee, and A. P. Lightman, 1973, Phys. Rev. D 8, 3308.

Eardley, D. M., D. L. Lee, A. P. Lightman, R. V. Wagoner, and C. M. Will, 1973, Phys. Rev. Lett. 30, 884.

Edmonds, A. R., 1957, Angular Momentum in Quantum Mechanics (Princeton University, Princeton, N.J.).

Einstein, A., 1918, Preuss. Akad. Wiss. Berlin, Sitzber. 1918, 154.

Epstein, R., and R. V. Wagoner, 1975, Astrophys. J. 197, 717.

Fock, V., 1964, The Theory of Space, Time, and Gravitation, second revised edition (Macmillan, New York).

Gel'fand, I. M., R. A. Minlos, and Z. Ya. Shapiro, 1963, Representations of the Rotation and Lorentz Groups (Pergamon, Oxford).

Geroch, R., 1970, J. Math. Phys. 11, 2580.

Goldberg, J. N., A. J. MacFarlane, E. T. Newman, F. Rorlich, and E. C. G. Sudarshan, 1967, J. Math. Phys. 8, 2155.

Hansen, R. O., 1974, J. Math. Phys. 15, 46. Hernandez, W. C., 1967, Phys. Rev. 159, 1070. Hoenselaers, C., W. Kinnersley, and B. C. Xanthopoulos, 1979, Phys. Rev. Lett. 42, 481.

Ipser, J. R., 1970, Astrophys. J. 166, 175.

Isaacson, R. A., 1968, Phys. Rev. 166, 1272. 
Janis, A. I., and E. T. Newman, 1965, J. Math. Phys. 6, 902 .

Lamb, D. J., 1966, J. Math. Phys. 7, 458.

Landau, L. D., and E. M. Lifshitz, 1941, Teoriya Polya (Nauka, Moscow) [English translation of a later edition, The Classical Theory of Fields (Addison-Wesley, Cambridge, Mass. (1951)].

Landau, L. D., and E. M. Lifshitz, 1971, The Classical Theory of Fields, third English edition (Pergamon, Oxford). Mashoon, B., 1973, Astrophys. J. Lett. 181, L65.

Mathews, J., 1962, J. Soc. Ind. Appl. Math. 10, 768.

Misner, C. W., K. S. Thorne, and J. A. Wheeler, 1970, Gravitation, first preliminary edition (University of Maryland, College Park).

Misner, C. W., K. S. Thorne, and J. A. Wheeler, 1971, Gravitation, second preliminary edition (Caltech, Pasadena); reprinted by the University of Utah, Salt Lake City, 1972.

Misner, C. W., K. S. Thorne, and J. A. Wheeler, 1973, Gravitation (Freeman, San Francisco); cited in text as MTW. Morgan, T., 1971, Phys. Rev. D 3, 800.

Muller zum Hagen, H., 1970, Proc. Cambridge Philos. Soc. $68,199$.

Newman, E. T., and R. Penrose, 1962, J. Math. Phys. 3, $566 ; 4$, $998(\mathrm{E})$.

Newman, E. T., and R. Penrose, 1966, J. Math. Phys. 7, 863.

Newman, E. T., and R. Penrose, 1968, Proc. R. Soc. London Ser. A 305, 175.

Papapetrou, A., 1962, C. R. Acad. Sci. Paris 255, 1578.

Papapetrou, A., 1971, Ann. Inst. Henri Poincare 14, 79.

Penrose, R., 1964, in Relativity, Groups, and Topology, edited by C. and B. DeWitt (Gordon and Breach, New York).

Penrose, R., 1968, in Battelle Rencontres: 1967 Lectures in Mathematics and Physics, edited by C. DeWitt and J. A. Wheeler (Benjamin, New York).

Peres, A., 1962, Phys. Rev. 128, 2471.

Peres, A., and N. Rosen, 1960, Ann. Phys. (N. Y.) 10, 94. Peters, P. C., 1964, Phys. Rev. 136, B1224.

Peters, P. C., and J. Mathews, 1963, Phys. Rev. 131, 435.

Press, W. H., and K. S. Thorne, 1972, Ann. Rev. Astron.
Astrophys. 10, 335.

Price, R. H., 1972, Phys. Rev. D 10, 2419.

Pirani, F. A. E., 1964, in Lectures on General Relativity, edited by A. Trautman, F. A. E. Pirani, and H. Bondi (Prentice-Hall, Englewood Cliffs).

Regge, T. and J. A. Wheeler, 1957, Phys. Rev. 108, 1063. Rose, M. E., 1955, Multipole Fields (Wiley, New York). Sandberg, V. D., 1978, J. Math. Phys. 19, 2441.

Sachs, R., 1961, Proc. R. Soc. London Ser. A 264, 309; especially Appendix B.

Sachs, R., 1962, Proc. R. Soc. London Ser. A 270, 103.

Sachs, R., 1964, in Relativity, Groups, and Topology,

edited by B. and C. DeWitt (Gordon and Breach, New York).

Sachs, R. and P. G. Bergmann, 1958, Phys. Rev. 112, 674.

Schumaker, B. L., and K. S. Thorne, 1980, paper in preparation.

Thorne, K. S., 1972, in Magic Without Magic: John Archibald Wheeler, edited by J. Klauder (Freeman, San Francisco).

Thorne, K. S., 1977, in Topics in Theoretical and Experimental Gravitation Physics, edited by V. De Sabbata and

J. Weber (Plenum, London).

Thorne, K. S., 1980, Mon. Not. Roy. Astron. Soc., in press.

Thorne, K. S. and S. J. Kovács, Jr., 1975, Astrophys. J. $200,245$.

Turner, M., 1977, Astrophys. J. 216, 914.

Turner, M. and R. V. Wagoner, 1979, in Sources of Gravitational Radiation, edited by L. Smarr (Cambridge University, Cambridge, England).

van der Burg, M. G. J., 1968, Proc. R. Soc. London Ser. A $303,37$.

Wagoner, R. V., 1977, in Gravitazione Sperimentale, Proceedings of an international symposium held at Pavia, Italy, 17-20 September 1976, edited by B. Bertotti (Accademia Nazionale dei Lincei, Rome).

Wagoner, R. V., 1979, Phys. Rev. D 19, 2897.

Xanthopoulos, B. C., 1979, J. Phys. A(GB) 12, 1025.

Zerilli, F. J., 1969, unpublished Ph. D. thesis, Princeton University.

Zerilli, F. J., 1970, J. Math. Phys. 11, 2203. 